Relative Abundance and Distribution of Fishes and Crayfish at Ash Meadows National Wildlife Refuge, Nye County, Nevada, 2007-08

Open-File Report 2011-1017 



\section{Relative Abundance and Distribution of Fishes and Crayfish at Ash Meadows National Wildlife Refuge, Nye County, Nevada, 2007-08}

By G. Gary Scoppettone, Peter Rissler, Danielle Johnson, and Mark Hereford

Open-File Report 2011-1017

U.S. Department of the Interior

U.S. Geological Survey 


\section{U.S. Department of the Interior \\ KEN SALAZAR, Secretary}

U.S. Geological Survey
Marcia K. McNutt, Director

U.S. Geological Survey, Reston, Virginia: 2011

For more information on the USGS-the Federal source for science about the Earth, its natural and living resources, natural hazards, and the environment, visit http://www.usgs.gov or call 1-888-ASK-USGS.

For an overview of USGS information products, including maps, imagery, and publications, visit $h$ ttp://www.usgs.gov/pubprod

To order this and other USGS information products, visit http://store.usgs.gov

Suggested citation:

Scoppettone, G.G., Rissler, Peter, Johnson, Danielle, and Hereford, Mark, 2011, Relative abundance and distribution of fishes and crayfish at Ash Meadows National Wildlife Refuge, Nye County, Nevada, 2007-08: U.S. Geological Survey Open-File Report 2011-1017, 56 p.

Any use of trade, product, or firm names is for descriptive purposes only and does not imply endorsement by the U.S. Government.

Although this report is in the public domain, permission must be secured from the individual copyright owners to reproduce any copyrighted material contained within this report. 


\section{Contents}

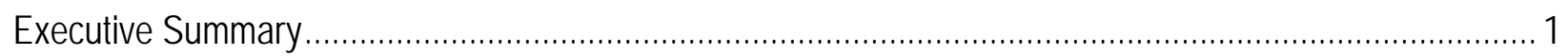

Introduction

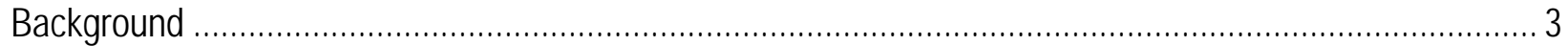

Habitat Alteration and Water Manipulation .................................................................................... 3

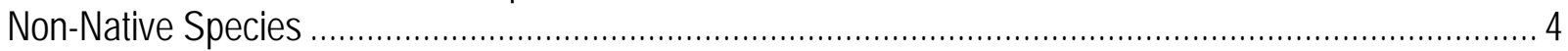

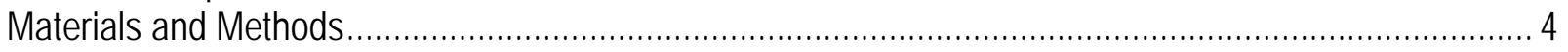

Results

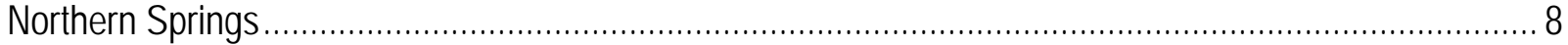

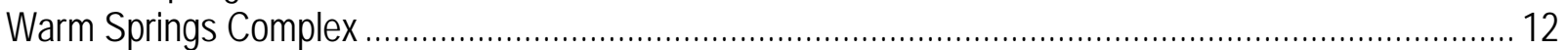

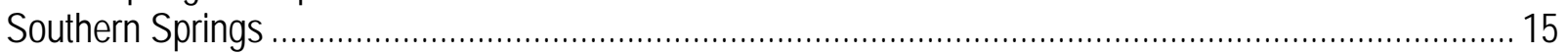

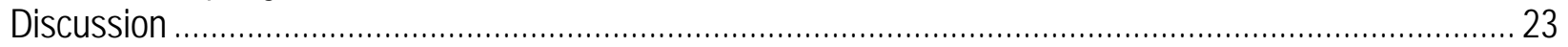

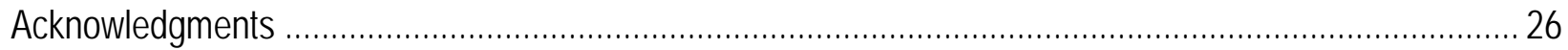

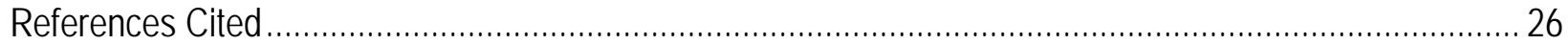

Appendix A. Seasonal Distributions of Fishes and Crayfish at Ash Meadows National Wildlife Refuge,

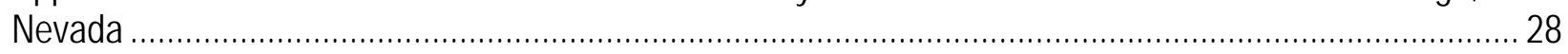

Appendix B. Seasonal Distributions of Fishes and Crayfish at Warm Springs Complex, Ash Meadows

National Wildlife Refuge, Nevada ................................................................................................. 37

Appendix C. Seasonal Distributions of Fishes and Crayfish in Southern Springs, Ash Meadows National

Wildlife Refuge, Nevada .......................................................................................................... 43

\section{Figures}

Figure 1. Aerial photograph of Ash Meadows National Wildlife Refuge showing 853 potential sampling stations, Nye County, Nevada.

Figure 2. Aerial photograph of Ash Meadows National Wildlife Refuge showing spring outflows and modeled historical outflows using 3- and 10-meter Digital Elevation Models (DEM) of Ash Meadows National Wildlife Refuge, Nevada.

Figure 3. Relative abundance and distribution of Ash Meadows Amargosa pupfish in the northern springs, Ash Meadows National Wildlife Refuge, Nevada, fall 2007-summer 2008

Figure 4. Relative abundance and distribution of Warm Springs Amargosa pupfish in the Warm Springs

Complex, Ash Meadows National Wildlife Refuge, Nevada, fall 2007-summer 2008.

Figure 5. Kemel density estimations (50 and 95 percent probability) of Warm Springs Amargosa

pupfish in the Warm Springs Complex, Ash Meadows National Wildlife Refuge, Nevada,

fall 2007-summer 2008

Figure 6. Relative abundance and distribution of Ash Meadows Amargosa pupfish in the southern springs, Ash Meadows National Wildlife Refuge, Nevada, fall 2007-summer 2008

Figure 7. Relative abundance and distribution of Ash Meadows speckled dace in the southern springs, Ash Meadows National Wildlife Refuge, Nevada, fall 2007-summer 2008.

Figure 8. Kemel density estimations (50 and 95 percent probability) of Ash Meadows Amargosa

pupfish in King's Pool, Point of Rocks, and Jackrabbit Springs, Ash Meadows National Wildlife Refuge, Nevada, fall 2007-summer 2008.

Figure 9. Kemel density estimations (50 and 95 percent probability) of Ash Meadows speckled dace in Jackrabbit Spring, Ash Meadows National Wildlife Refuge, Nevada, fall 2007-summer 2008 .................. 22

springs, Ash Meadows National Wildlife Refuge, Nevada, fall 2007-summer 2008. ................................. 44 


\section{Tables}

Table 1.Seasonal catches of fishes, bullfrogs, and crayfish at the northern springs of the Ash Meadows National Wildlife Refuge, Nevada, fall 2007-summer 2008

Table 2. Seasonal catches of fishes, bullfrogs, and crayfish at the Warm Springs Complex of the Ash Meadows NWR, Nevada, fall 2007-summer 2008.

Table 3. Seasonal catches of fishes, bullfrogs, and crayfish at the southern springs of the Ash Meadows National Wildlife Refuge, Nevada, fall 2007-summer 2008

\section{Conversion Factors}

\begin{tabular}{lll}
\hline \multicolumn{1}{c}{ Multiply } & \multicolumn{1}{c}{ By } & To obtain \\
\hline centimeter (cm) & 0.3937 & inch (in.) \\
hectare (ha) & 2.471 & acre \\
millimeter (mm) & 0.03937 & inch (in.) \\
meter (m) & 1.094 & yard (yd) \\
liter per minute (L/min) & 0.26417 & gallon per minute (gal/min) \\
\hline
\end{tabular}

Temperature in degrees Celsius $\left({ }^{\circ} \mathrm{C}\right)$ may be converted to degrees Fahrenheit $\left({ }^{\circ} \mathrm{F}\right)$ as follows:

$$
{ }^{\circ} \mathrm{F}=\left(1.8 x^{\circ} \mathrm{C}\right)+32 \text {. }
$$




\section{Relative Abundance and Distribution of Fishes and Crayfish at Ash Meadows National Wildlife Refuge, Nye County, Nevada, 2007-08}

By G. Gary Scoppettone, Peter Rissler, Danielle Johnson, and Mark Hereford

\section{Executive Summary}

This study provides baseline data of native and non-native fish populations in Ash Meadows National Wildlife Refuge (NWR), Nye County, Nevada, that can serve as a gauge in native fish enhancement efforts. In support of Carson Slough restoration, comprehensive surveys of Ash Meadows NWR fishes were conducted seasonally from fall 2007 through summer 2008. A total of 853 sampling stations were created using Geographic Information Systems and National Agricultural Imagery Program. In four seasons of sampling, Amargosa pupfish (genus Cyprinodon) was captured at 388 of 659 stations. The number of captured Amargosa pupfish ranged from 5,815 (winter 2008) to 8,346 (summer 2008). The greatest success in capturing Amargosa pupfish was in warm water spring-pools with temperature greater than $25^{\circ} \mathrm{C}$, headwaters of warm water spring systems, and shallow (depths less than 10 centimeters) grassy marshes. In four seasons of sampling, Ash Meadows speckled dace (Rhinichthys osculus nevadesis) was captured at 96 of 659 stations. The number of captured Ash Meadows speckled dace ranged from 1,009 (summer 2008) to 1,552 (winter 2008). The greatest success in capturing Ash Meadows speckled dace was in cool water spring-pools with temperature less than $20^{\circ} \mathrm{C}$ and in the high flowing water outflows. Among 659 sampling stations within the range of Amargosa pupfish, red swamp crayfish (Procambarus clarkii) was collected at 458 stations, western mosquitofish (Gambusia affinis) at 374 stations, and sailfin molly (Poecilia latipinna) at 128 stations. School Springs was restored during the course of this study. Prior to restoration of School Springs, maximum Warm Springs Amargosa pupfish (Cyprinodon nevadensis pectoralis) captured from the six springs of the Warm Springs Complex was 765 (fall 2007). In four seasons of sampling, Warm Springs Amargosa pupfish were captured at 85 of 177 stations. The greatest success in capturing Warm Springs Amargosa pupfish when co-occurring with red swamp crayfish and western mosquitofish was in water with temperature greater than $26^{\circ} \mathrm{C}$ near the springhead, and in shallow (depths less than 10 centimeters) grassy marshes. Among 177 sampling stations within the range of Warm Springs Amargosa pupfish, red swamp crayfish were collected at 96 stations and western mosquitofish were collected at 49 stations. Removal of convict cichlid (Amatitlania nigrofasciata) from Fairbanks Spring was followed by a substantial increase in Ash Meadows Amargosa pupfish (Cyprinodon nevadensis mionectes) captures from 910 pre-removal to 3,056 post-removal. Red swamp crayfish was continually removed from 
Bradford 1 Spring, which seemed to cause an increase in the speckled dace population.

Restoration of Kings Pool and Jackrabbit Springs promoted the success of native fishes with the greatest densities in restored reaches. Ongoing restoration of Carson Slough and its tributaries, as well as control and elimination of invasive species, is expected to increase abundance and distribution of Ash Meadows' native fish populations. Further analysis of data from this study will help determine the habitat characteristic(s) that promote native species and curtail nonnative species.

\section{Introduction}

The 2007 and 2008 seasonal surveys of this study focused on abundance and distribution of fishes within the boundary of the Ash Meadows National Wildlife Refuge (NWR). This effort was intended to provide baseline information as part of the Carson Slough restoration. Historically, most Ash Meadows NWR spring systems were connected (directly or indirectly; perennially or intermittently) to Carson Slough (Miller, 1948). Thus, slough restoration includes reconnection of tributaries, which in turn necessitates a comprehensive survey of Ash Meadows endangered fish populations to comply with the National Environmental Policy Act (U.S. Environmental Protection Agency, 2010) and Endangered Species Act (U.S. Department of the Interior, 1973). This study is intended to fulfill the requirements of these two Federal acts. The first published Ash Meadows fishes survey was conducted by Miller (1948), who described Warm Springs Amargosa pupfish (Cyprinodon nevadensis pectoralis) (CYPE), Ash Meadows Amargosa pupfish (Cyprinodon nevadensis mionectes) (CYMI), and revisited the description of the now extinct Ash Meadows poolfish (Empetrichthys merriami). Miller (1948) briefly describes the springs these fishes occupied along with cohabitants, native Ash Meadows speckled dace [Rhinichthys osculus nevadesis (Hubbs and Deacon, 1964; Miller, 1948)] (RHON) and non-native western mosquitofish (Gambusia affinis) (GAAF). Hardy (1979) reported on the relative abundance and distribution of fishes in most the Ash Meadows NWR spring systems, but his observations were qualitative as were those of Threloff (1990). Scoppettone and others (1995) seasonally conducted an abundance and distribution study of Ash Meadows NWR fishes, but did not include all Ash Meadows NWR spring systems. Other publications and reports on distributions pertained to new localities (Miller and Deacon, 1973; Baugh and others, 1986) and publications and reports on abundance typically focused on spring-pools (Williams and Sada, 1985). Because the Ash Meadows NWR's water resources are so extensive, no comprehensive and systematic survey has been completed of Ash Meadows NWR fishes. In this study, we monitored the relative abundance and distribution of fishes and crayfish of the Ash Meadows NWR. Crayfish were included because these fishes seem to negatively affect native fish populations. Each sampling location is described and habitat conditions are quantified. 


\section{Background}

Native fish abundance and distribution at Ash Meadows have been negatively affected by a long history of spring system alteration, water manipulation, and introduction of non-native aquatic species (Miller, 1948; Pister, 1974; Dudley and Larson, 1976; Soltz and Naiman, 1978; Sada, 1990). Habitat and water manipulation for agriculture and municipal use ceased with the establishment of the Ash Meadows NWR, but eroding irrigation channels, habitat improvement projects, and continued presence of invasive aquatic species caused ongoing changes in native fish populations that masked fluctuations in natural populations (Williams and others, 2001). Documentation of recent human induced habitat alterations was used in the interpretation of our results.

\section{Habitat Alteration and Water Manipulation}

Habitat alteration and water manipulation of Ash Meadows springs for agricultural purposes began in the 19th century (Rectangular Survey Plat Map, December 1881 and January 1882), was intensive in the 1970s (Soltz and Naiman, 1978; Sada, 1990; Deacon and Williams, 1991), and ended when the Ash Meadows NWR was established in 1984. By that time, Ash Meadows was incorporated into the National Wildlife Refuge system, many of the springheads had been enlarged, and discharge was conveyed in excavated earthen and concrete ditches. Although native fish habitat had been greatly altered, the virtual halt to diversion of water for irrigation provided fish a more stable environment with water and fish no longer being lost to irrigated fields. Presumably, there was a subsequent increase in the number of Amargosa pupfish (genus Cyprinodon) and Ash Meadows speckled dace.

Since 1984 when the Ash Meadow NWR was established, local habitat and water manipulation projects generally focused on enhancement of native fish populations. In the early 1990s, Lower Crystal Marsh was created by breaching the southeastern dike of Horseshoe Reservoir, and was further modified in the 1990s. This action was intended to expand the wetland to benefit Ash Meadows Amargosa pupfish (Threloff, 1990). Dry headwater marshes (water breached unmaintained excavated earthen or concrete irrigation ditches) were created by directing local flow into a stream channel. For example, in 1997, Kings Pool spring-pool was reduced in size to the presumed original size and the meandering stream channel was excavated (Gourley and Ammon, 1997); this action had a positive effect on the Ash Meadows Amargosa pupfish population (Scoppettone and others, 2005). In the mid-1990s, about $70 \mathrm{~m}$ of Fairbanks Spring outflow was trenched $300 \mathrm{~m}$ downstream of the spring-pool to prevent water from flooding the road. Although inadvertent, this action was presumed to improve Ash Meadows Amargosa pupfish habitat. Restoration also was implemented at Point-of-Rocks Spring in 2001 and at the upper and lower parts of Jackrabbit Spring outflow channel in the summers of 2006 and 2007, respectively. During the course of this study, School Spring was rehabilitated from a series of concrete pools (constructed in the 1970s and 1980s) to a flowing stream with small pools terminating in a marsh. 


\section{Non-Native Species}

The introduction of several non-native species has contributed to the decline of Ash Meadows native fish (Williams and Deacon, 1986; Sada, 1990; Kennedy and others, 2006). Mosquitofish was established by the 1930s and was the first reported introduction of a nonnative species (Miller, 1948). Sailfin molly (Poecilia latipinna) (POLA) was established by the early 1960s (La Rivers, 1962; Deacon and others, 1964; Hubbs and Deacon, 1964); several other tropical fishes appeared in Ash Meadows waters about the same time, but these tropical fishes did not establish a reproductive population (Soltz and Naiman, 1978). A population of the tropical convict cichlid (Archocentrus nigrofasciatus) (ARNI) was discovered in Fairbanks spring-pool in October 2001 (Shawn Goodchild, U.S. Fish and Wildlife Service, oral commun., 2001). Largemouth bass (Micropterus salmoides) (MISA) has been present in the Ash Meadows spring systems since the 1960s (Williams and Deacon, 1986; Sada, 1990) and since 1995, have established in Point-of-Rocks, King's Pool, Forest, Big, Bradford 2, and Crystal Springs. Green sunfish (Lepomis cyanellus) (LECY) appeared in Crystal Reservoir in the 1990s, and in Bradford 2 Spring by 2001 (Leavy and others, 2004). A reproductive population of black bullhead (Ameiurus melas) (AMME) has occupied Davis Spring; chemical removal of this population was attempted in the1990s and again in July 2008 (Darrick Weissenfluh, Ash Meadows National Wildlife Refuge, oral commun., 2008).

Several largemouth bass in Big Spring and an apparent invasion of bass and green sunfish into the inflow of Crystal Reservoir were documented during this study. In January 2008, convict cichlids were chemically removed from Fairbanks Spring (Darrick Weissenfluh, Ash Meadows National Wildlife Refuge, oral commun., 2008). Red swamp crayfish (Procambarus clarkii) (PRCL) and American bullfrog (Lithobates catesbeiana) (LICA) have been established since the early 1960s (Sada, 1990). The exact date of introduction is unknown.

\section{Materials and Methods}

We used Geographic Information Systems (GIS) using the National Agricultural Imagery Program (NAIP) to trace stream channels and to determine locations of sampling stations. A total of 853 sampling locations were established, but the number of locations with water sufficiently deep to trap fish ranged from 781 in fall 2007 to 631 in summer 2008 (fig. 1). North American Datum (NAD) 83 decimal degrees of longitude and latitude of each station were downloaded into Garmin Global Positioning System (GPS) units. These units typically are accurate within a 2 $\mathrm{m}$ radius of a specific station. GIS also was used to illustrate seasonal distribution and relative abundance of fishes in each spring system. The data collected during these seasonal sampling periods were used to develop species specific GIS maps, which display range and densities. Where it was feasible (relatively long outflows and some marshes), a fixed kernel methodology was used (Worton, 1987) to focus on areas of greatest concentration of fish population. A 95 percent kernel was the minimum area in which 95 percent of the population occupied, and 50 percent kernel was the minimum area in which 50 percent of the population occupied. ArcView GIS and Animal Movement Extension (Hooge and Eichenlaub, 1997) were used to estimate kernel densities. 


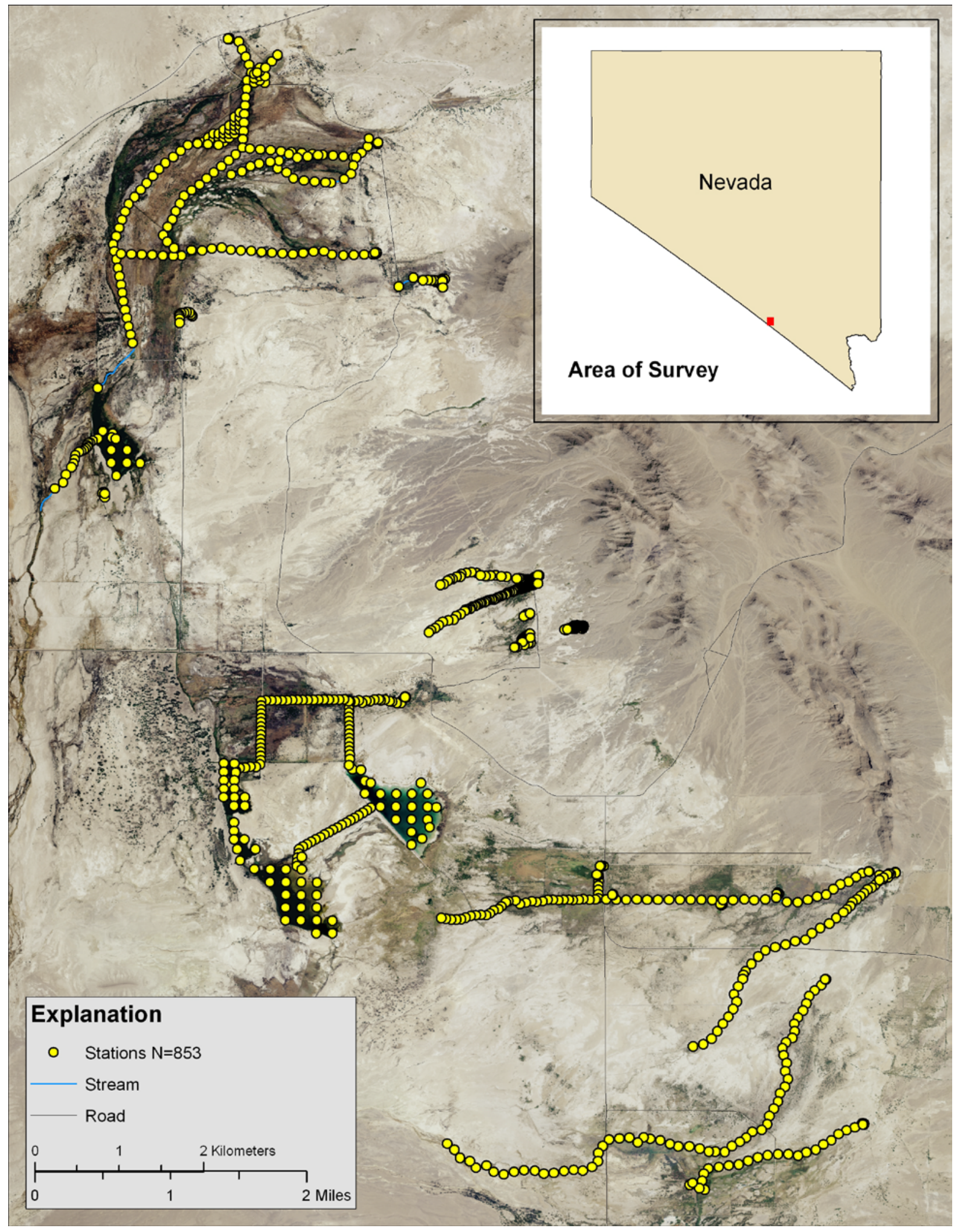

Figure 1. Aerial photograph of Ash Meadows National Wildlife Refuge showing 853 potential sampling stations, Nye County, Nevada. 
Most traps were set in the afternoon and pulled the following morning. Shorter sets, placed in the morning and pulled 3-9.5 hours later, were used in areas with high fish densities, high crayfish densities, spring pools, and areas of relatively warm water with temperatures greater then $29^{\circ} \mathrm{C}$ to prevent fish mortality. Each station was sampled for all four seasons unless precluded by restoration projects, water level too low for successful fish capture, or water velocity to fast for traps to remain. Small minnow traps (90 $\mathrm{mm}$ in diameter) were fabricated to sample shallow areas and were effective in depths to $60 \mathrm{~mm}$. Entries for standard and fabricated minnow traps was $20 \mathrm{~mm}$ in diameter, trap walls were made up of a $3.3 \mathrm{~mm}$ mesh. Standard minnow traps allow fish entry from two sides and fabricated traps allow fish entry from one side. Traps were baited with dry dog food. At each station, fish were identified to species, counted, and 10-20 individuals were randomly selected and measured to fork length (FL). The capture gear and seasonal effort used at each system are presented in the first table of each of the three regional appendixes (appendixes A-C).

Water-quality data $(\mathrm{pH}$, temperature, dissolved oxygen, conductivity, and depth) were collected at each station using HydroLab MS5 or other equivalent water-sampling equipment. Water depth was measured at or near the site of minnow trap placement where possible. Flow was measured in fall 2009 with a Marsh McBirney Model 201 portable water current meter. Vegetation and substrate surveys were completed in spring 2008 and winter 2009, and were comprised of species-specific cover estimates of canopy, aquatic surface, and subsurface aquatic vegetation densities.

We used Program ArcGIS 9.3's ArcHydro Tools 9 to illustrate the relation of Ash Meadows spring outflows to historical Carson Slough and 3- and 10-m Digital Elevation Models (DEM) as a base layer for historical watercourse analysis (fig. 2). Alternate historical flows were modeled in cases where terrain structures could cause water to flow in multiple directions. The current and modeled historical outflows shown in figure 2 will aid in interpretation of fish and crayfish distribution.

Relative abundance and distribution are illustrated using three methods-(1) total fish captured in each system and major habitat type (springhead, spring-pool, outflow, marsh, and reservoir) is shown by season in tabular form, (2) distribution and abundance (combined seasonal data to streamline this report) are presented using GIS-generated maps, and (3) kernel densities are estimated based on selected systems for describing seasonal distribution. For ease of reporting, Ash Meadows NWR spring systems were divided into three different regions: northern springs, Warm Springs Complex, and southern springs. 


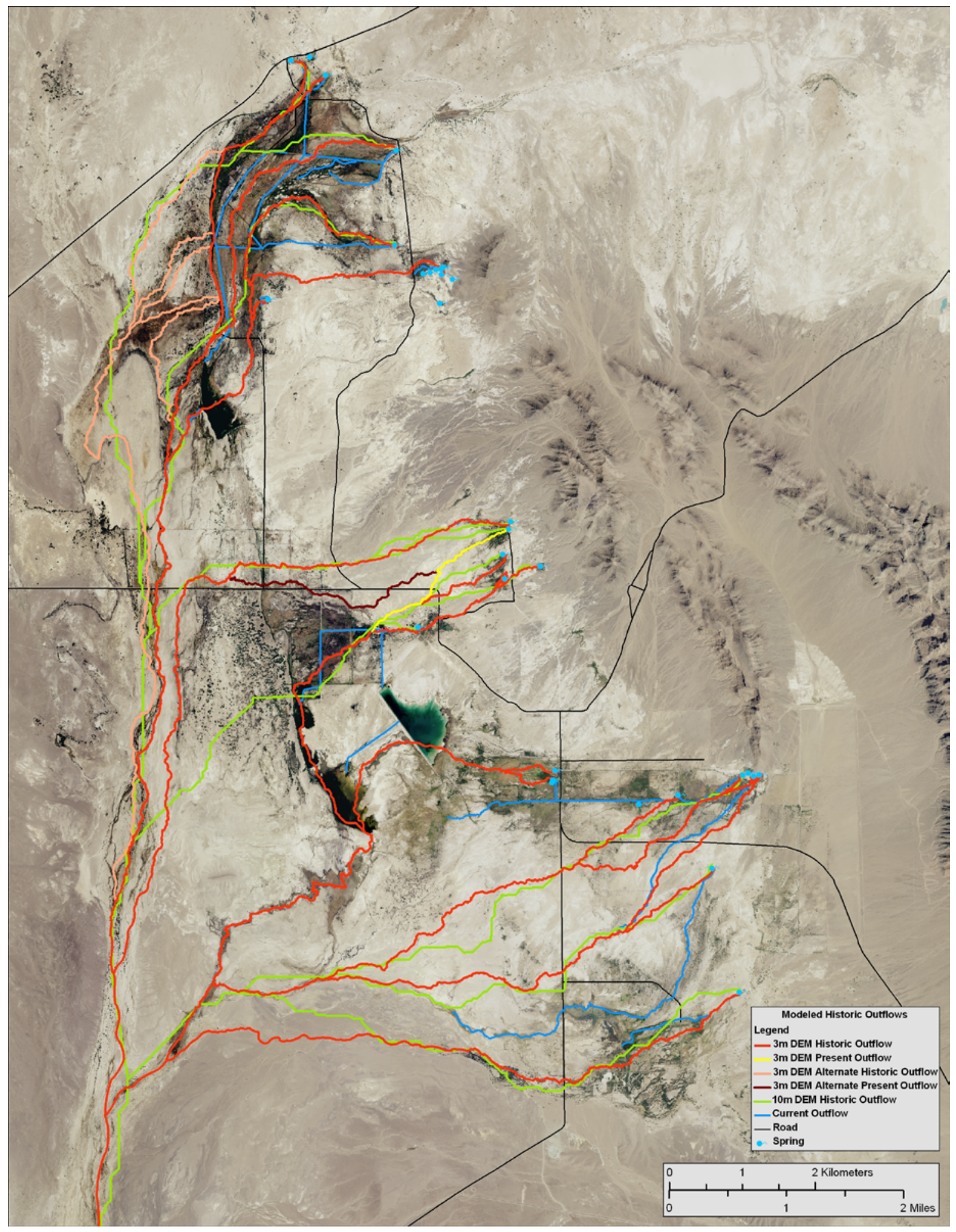

Figure 2. Aerial photograph of Ash Meadows National Wildlife Refuge showing spring outflows and modeled historical outflows using 3- and 10-meter Digital Elevation Models (DEM) of Ash Meadows National Wildlife Refuge, Nevada. 


\section{Results}

\section{Northern Springs}

The greatest Ash Meadows Amargosa pupfish density in Fairbanks, Rogers, and Longstreet Springs was in the spring-pool, as was true of most Ash Meadows large warm water spring systems (fig. 3, table 1). Total capture of Ash Meadows Amargosa pupfish from Rogers and Longstreet Springs was greater in the spring-pool than the entire outflow. Longstreet Spring was the only northern spring harboring sailfin molly, and total number and catch rate were greatest in the spring-pool (appendix A: table A1, fig. A2). The greatest number of bullfrog tadpole captures was from Rogers Spring, and most were from the spring-pool in each of the four seasonal surveys.

Western mosquitofish were the only minnow trap captures from Soda Spring (fall 2007); crayfish were captured in all four seasonal surveys (table 1, appendix A: figs. A1 and A4). Our captures suggest Cold Spring probably is the only Ash Meadows spring where Ash Meadows Amargosa pupfish is not sympatric with mosquitofish.

After chemical removal of convict cichlids and mosquitofish in Fairbanks spring-pool and part of the outflow in January 2008, Ash Meadows pupfish captures markedly increased. In Fairbanks spring-pool, captures per trap almost doubled from a total capture of 453 Ash Meadows Amargosa pupfish (75.5 / trap) in fall 2007 to 821 captures (136.8 / trap) in summer 2008 (appendix A: table A1). Prior to treatment, convict cichlids were concentrated in the upper $300 \mathrm{~m}$ of the stream but several were captured as far as 1,500 $\mathrm{m}$ downstream (appendix A: fig. A3). Water temperatures at the time of capture ranged from 25.5 to $27.5^{\circ} \mathrm{C}$. 


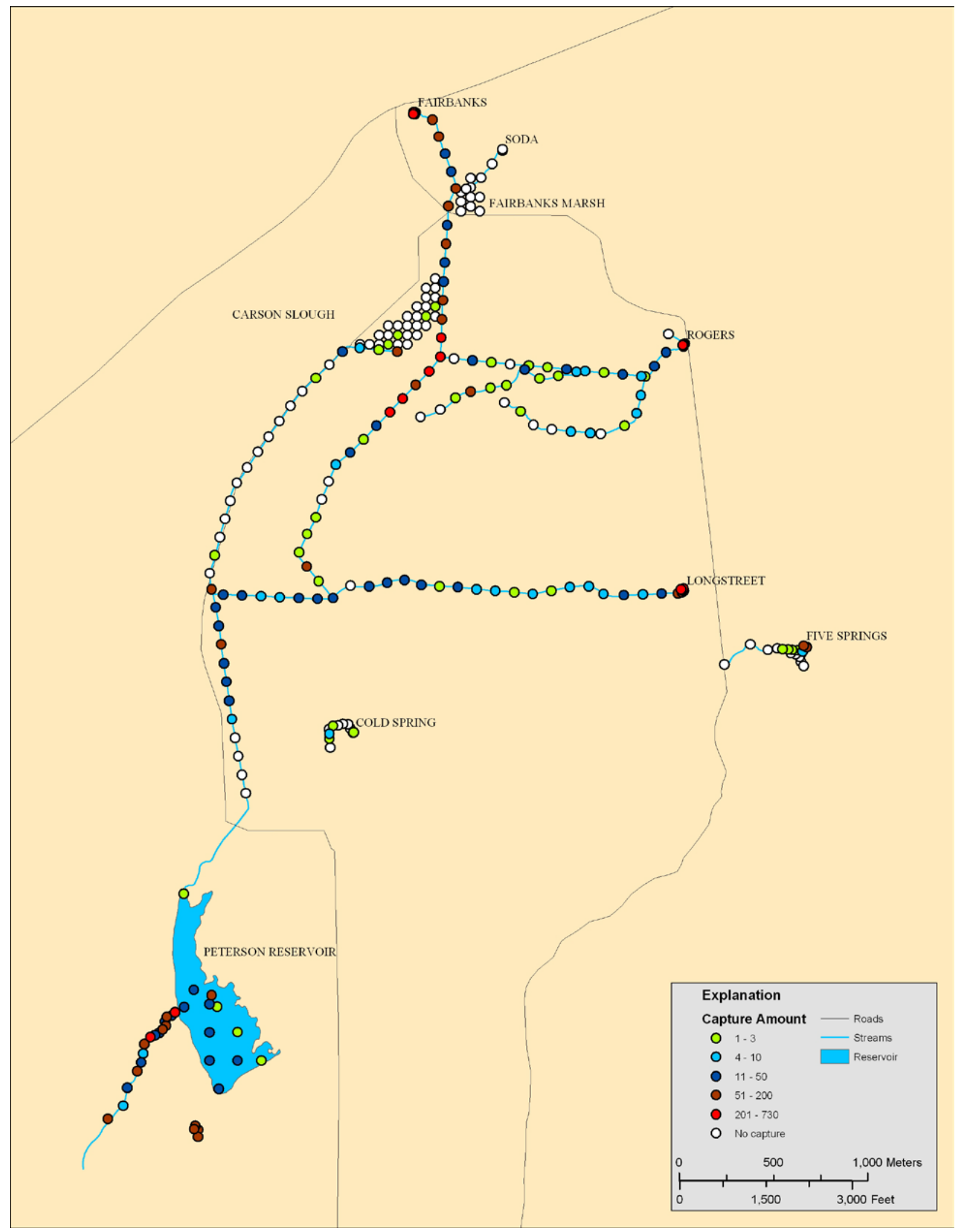

Figure 3. Relative abundance and distribution of Ash Meadows Amargosa pupfish in the northern springs, Ash Meadows National Wildlife Refuge, Nevada, fall 2007-summer 2008. 
Table 1. Seasonal catches of fishes, bullfrogs, and crayfish at the northern springs of the Ash Meadows National Wildlife Refuge, Nevada, fall 2007-summer 2008.

[Spring locations are shown in figures 1 and 2. CYMI, Ash Meadows Amargosa pupfish; CYPE, Warm Springs Amargosa pupfish; RHON, Ash Meadows speckled dace; GAAF, Mosquitofish; POLA, Sailfin Molly; LECY, Green Sunfish; MISA, Largemouth Bass; ARNI, Convict Cichlid; AMME, Black Bullhead; LICA, Bull Frog; PRCL, Red Swamp Crayfish]

\begin{tabular}{|c|c|c|c|c|c|c|c|c|c|c|c|c|}
\hline System & Season & CYMI & CYPE & RHON & GAAF & POLA & LECY & MISA & ARNI & AMME & LICA & PRCL \\
\hline \multirow{4}{*}{$\begin{array}{l}\text { Fairbanks } \\
\text { spring-pool }\end{array}$} & Fall 07 & 453 & - & - & 1 & - & - & - & 50 & - & 5 & 4 \\
\hline & Winter 08 & 581 & - & - & - & - & - & - & - & - & - & 18 \\
\hline & Spring 08 & 358 & - & - & - & - & - & - & - & - & - & 21 \\
\hline & Summer 08 & 821 & - & - & - & - & - & - & - & - & - & 35 \\
\hline \multirow{4}{*}{$\begin{array}{l}\text { Fairbanks } \\
\text { stream }\end{array}$} & Fall 07 & 457 & - & - & 200 & - & - & - & 72 & - & 4 & 144 \\
\hline & Winter 08 & 149 & - & - & - & - & - & - & - & - & - & 131 \\
\hline & Spring 08 & 284 & - & - & 2 & - & - & - & - & - & 7 & 196 \\
\hline & Summer 08 & 2235 & - & - & 45 & - & - & - & - & - & - & 295 \\
\hline \multirow{3}{*}{$\begin{array}{l}\text { Fairbanks } \\
\text { marsh }\end{array}$} & Fall 07 & - & - & - & - & - & - & - & - & - & - & 2 \\
\hline & Winter 08 & - & - & - & - & - & - & - & - & - & - & - \\
\hline & Spring 08 & - & - & - & - & - & - & - & - & - & - & 6 \\
\hline $\begin{array}{l}\text { Carson } \\
\text { marsh }\end{array}$ & Fall 07 & 4 & - & - & 11 & - & - & - & - & - & - & 50 \\
\hline \multirow{4}{*}{$\begin{array}{c}\text { Soda } \\
\text { spring-pool }\end{array}$} & Fall 07 & - & - & - & 8 & - & - & - & - & - & - & 12 \\
\hline & Winter 08 & - & - & - & - & - & - & - & - & - & - & 18 \\
\hline & Spring 08 & - & - & - & - & - & - & - & - & - & - & 13 \\
\hline & Summer 08 & - & - & - & - & - & - & - & - & - & - & 16 \\
\hline \multirow{4}{*}{$\begin{array}{l}\text { Soda } \\
\text { stream }\end{array}$} & Fall 07 & - & - & - & 3 & - & - & - & - & - & - & 12 \\
\hline & Winter 08 & - & - & - & - & - & - & - & - & - & - & 5 \\
\hline & Spring 08 & - & - & - & - & - & - & - & - & - & - & 8 \\
\hline & Summer 08 & - & - & - & - & - & - & - & - & - & - & 3 \\
\hline \multirow{4}{*}{$\begin{array}{c}\text { Rogers } \\
\text { spring-pool }\end{array}$} & Fall 07 & 410 & - & - & 42 & - & - & - & - & - & 12 & 12 \\
\hline & Winter 08 & 466 & - & - & 69 & - & - & - & - & - & 29 & 27 \\
\hline & Spring 08 & 340 & - & - & 39 & - & - & - & - & - & 9 & 31 \\
\hline & Summer 08 & 341 & - & - & 248 & - & - & - & - & - & 2 & 32 \\
\hline \multirow{4}{*}{$\begin{array}{l}\text { Rogers } \\
\text { stream }\end{array}$} & Fall 07 & 44 & - & - & 485 & - & - & - & - & - & 1 & 214 \\
\hline & Winter 08 & 59 & - & - & 123 & - & - & - & - & - & 1 & 227 \\
\hline & Spring 08 & 149 & - & - & 250 & - & - & - & - & - & 2 & 184 \\
\hline & Summer 08 & 60 & - & - & 919 & - & - & - & - & - & - & 144 \\
\hline \multirow{4}{*}{$\begin{array}{l}\text { Longstreet } \\
\text { spring-pool }\end{array}$} & Fall 07 & 546 & - & - & 56 & 53 & - & - & - & - & - & 17 \\
\hline & Winter 08 & 332 & - & - & 36 & 19 & - & - & - & - & - & 23 \\
\hline & Spring 08 & 353 & - & - & 1 & 45 & - & - & - & - & - & 23 \\
\hline & Summer 08 & 458 & - & - & 80 & 133 & - & - & - & - & - & 69 \\
\hline \multirow{4}{*}{$\begin{array}{l}\text { Longstreet } \\
\text { stream }\end{array}$} & Fall 07 & 3 & - & - & 7 & 2 & - & - & - & - & - & 33 \\
\hline & Winter 08 & 22 & - & - & 6 & 1 & - & - & - & - & - & 22 \\
\hline & Spring 08 & 95 & - & - & 39 & 5 & - & - & - & - & - & 53 \\
\hline & Summer 08 & 98 & - & - & 73 & 4 & - & - & - & - & - & 68 \\
\hline \multirow{4}{*}{$\begin{array}{c}\text { Five } \\
\text { Springs } \\
\text { spring-pool }\end{array}$} & Fall 07 & 32 & - & - & - & - & - & - & - & - & - & 2 \\
\hline & Winter 08 & 21 & - & - & 9 & - & - & - & - & - & - & 1 \\
\hline & Spring 08 & 23 & - & - & 5 & - & - & - & - & - & - & 5 \\
\hline & Summer 08 & 21 & - & - & 3 & - & - & - & - & - & - & - \\
\hline \multirow{4}{*}{$\begin{array}{c}\text { Five } \\
\text { Springs } \\
\text { stream }\end{array}$} & Fall 07 & 4 & - & - & 13 & - & - & - & - & - & - & 7 \\
\hline & Winter 08 & 3 & - & - & 57 & - & - & - & - & - & - & 20 \\
\hline & Spring 08 & 13 & - & - & 75 & - & - & - & - & - & - & 9 \\
\hline & Summer 08 & 46 & - & - & 68 & - & - & - & - & - & - & 41 \\
\hline
\end{tabular}


Table 1. Seasonal catches of fishes, bullfrogs, and crayfish at the northern springs of the Ash Meadows National Wildlife Refuge, Nevada, fall 2007-summer 2008.-Continued

\begin{tabular}{|c|c|c|c|c|c|c|c|c|c|c|c|c|}
\hline System & Season & CYMI & CYPE & RHON & GAAF & POLA & LECY & MISA & ARNI & AMME & LICA & PRCL \\
\hline \multirow{4}{*}{$\begin{array}{l}\text { Peterson } \\
\text { reservoir }\end{array}$} & Fall 07 & 44 & - & - & 2 & - & - & - & - & - & - & 3 \\
\hline & Winter 08 & 2 & - & - & - & - & - & - & - & - & - & 1 \\
\hline & Spring 08 & 12 & - & - & - & - & - & - & - & - & - & 4 \\
\hline & Summer 08 & 157 & - & - & 95 & - & - & - & - & - & - & 3 \\
\hline \multirow{4}{*}{$\begin{array}{l}\text { Peterson } \\
\text { stream }\end{array}$} & Fall 07 & 351 & - & - & 75 & - & - & - & - & - & - & 1 \\
\hline & Winter 08 & 108 & - & - & 5 & - & - & - & - & - & - & 28 \\
\hline & Spring 08 & 861 & - & - & 11 & - & - & - & - & - & - & 8 \\
\hline & Summer 08 & 509 & - & - & 993 & - & - & - & - & - & - & 20 \\
\hline \multirow{4}{*}{$\begin{array}{c}\text { Cold } \\
\text { spring-pool }\end{array}$} & Fall 07 & - & - & - & - & - & - & - & - & - & - & 17 \\
\hline & Winter 08 & - & - & - & - & - & - & - & - & - & - & 45 \\
\hline & Spring 08 & - & - & - & - & - & - & - & - & - & - & 32 \\
\hline & Summer 08 & - & - & - & - & - & - & - & - & - & - & 33 \\
\hline \multirow{4}{*}{ Cold pool } & Fall 07 & - & - & - & - & - & - & - & - & - & - & 25 \\
\hline & Winter 08 & - & - & - & - & - & - & - & - & - & - & 32 \\
\hline & Spring 08 & - & - & - & - & - & - & - & - & - & - & 18 \\
\hline & Summer 08 & - & - & - & - & - & - & - & - & - & - & 16 \\
\hline \multirow{4}{*}{$\begin{array}{l}\text { Cold } \\
\text { stream }\end{array}$} & Fall 07 & 3 & - & - & - & - & - & - & - & - & - & 39 \\
\hline & Winter 08 & - & - & - & - & - & - & - & - & - & - & 113 \\
\hline & Spring 08 & 4 & - & - & - & - & - & - & - & - & - & 54 \\
\hline & Summer 08 & - & - & - & - & - & - & - & - & - & - & 68 \\
\hline
\end{tabular}

Soda spring-pool water temperatures were cooler and fluctuated more than Fairbanks and Rogers spring-pools. Temperatures ranged from $19.1^{\circ} \mathrm{C}$ (winter 2008) to $26.2^{\circ} \mathrm{C}$ (summer 2008) in Soda, 26.9 to $28.5^{\circ} \mathrm{C}$ in Fairbanks, and 28.4 to $29.7^{\circ} \mathrm{C}$ in Rogers Springs. Longstreet springpool was slightly warmer than Soda spring-pool ranging from 19.9 to $28.8^{\circ} \mathrm{C}$, but also displayed a rather broad seasonal fluctuation. Five Springs and Cold Spring, like Soda Spring, have relatively low discharge $(<300 \mathrm{~L} / \mathrm{min})$ (Dudley and Larson, 1976). Five Springs are at the highest elevation $(715 \mathrm{~m})$ and were the warmest $\left(32-34^{\circ} \mathrm{C}\right)$. Cold Spring was the coolest $\left(18^{\circ} \mathrm{C}\right)$ of the northern springs. Ash Meadows Amargosa pupfish have been observed in Five Springs and Cold Spring. Five Springs captures were concentrated near the upper reaches and western mosquitofish was predominantly captured, although no mosquitofish were captured from Cold Spring. 


\section{Warm Springs Complex}

When this study began in fall 2007, four of the six springs of the Warm Springs Complex harbored crayfish and mosquitofish (North Indian, South Indian, South Scruggs, and School Springs). No Warm Springs Amargosa pupfish were captured or observed in South Indian Spring. In North Indian Spring, Warm Springs Amargosa pupfish captures ranged from 79 (fall 2007) to 34 (winter 2008), but were present only in the first $100 \mathrm{~m}$ of the outflow (fig. 4, table 2). Mosquitofish and crayfish were captured at all South Indian Spring trapping stations, although Warm Springs Amargosa pupfish were more localized (appendix B: table B1, figs. B1 and B2).

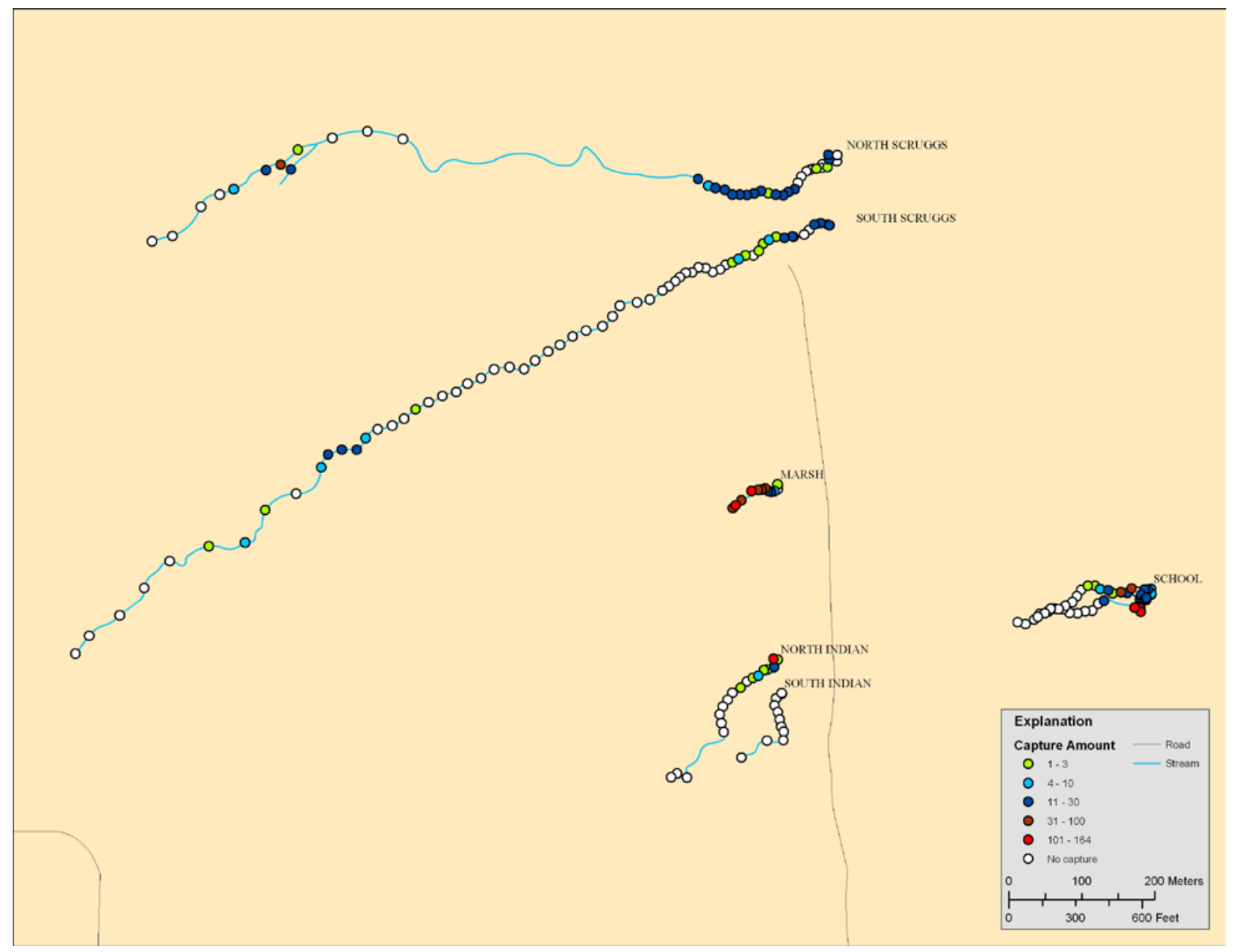

Figure 4. Relative abundance and distribution of Warm Springs Amargosa pupfish in the Warm Springs Complex, Ash Meadows National Wildlife Refuge, Nevada, fall 2007-summer 2008. 
Table 2. Seasonal catches of fishes, bullfrogs, and crayfish at the Warm Springs Complex of the Ash Meadows NWR, Nevada, fall 2007-summer 2008.

[Spring locations are shown in figures 1 and 2. CYMI, Ash Meadows Amargosa pupfish; CYPE, Warm Springs Amargosa pupfish; RHON, Ash Meadows speckled dace; GAAF, Mosquitofish; POLA, Sailfin Molly; LECY, Green Sunfish; MISA, Largemouth Bass; ARNI, Convict Cichlid; AMME, Black Bullhead; LICA, Bull Frog; PRCL, Red Swamp Crayfish]

\begin{tabular}{|c|c|c|c|c|c|c|c|c|c|c|c|c|}
\hline System & Season & CYMI & CYPE & RHON & GAAF & POLA & LECY & MISA & ARNI & AMME & LICA & PRCL \\
\hline \multirow{4}{*}{$\begin{array}{l}\text { North Scruggs } \\
\text { spring-pool }\end{array}$} & Fall 07 & - & - & - & - & - & - & - & - & - & - & - \\
\hline & Winter 08 & - & - & - & - & - & - & - & - & - & - & - \\
\hline & Spring 08 & - & 7 & - & - & - & - & - & - & - & - & - \\
\hline & Summer 08 & - & 16 & - & - & - & - & - & - & - & - & - \\
\hline \multirow{4}{*}{$\begin{array}{c}\text { North Scruggs } \\
\text { stream }\end{array}$} & Fall 07 & - & 86 & - & - & - & - & - & - & - & - & - \\
\hline & Winter 08 & - & 84 & - & - & - & - & - & - & - & - & - \\
\hline & Spring 08 & - & 81 & - & - & - & - & - & - & - & - & - \\
\hline & Summer 08 & - & 115 & - & - & - & - & - & - & - & - & - \\
\hline \multirow{4}{*}{$\begin{array}{l}\text { South Scruggs } \\
\text { spring-pool }\end{array}$} & Fall 07 & - & 17 & - & - & - & - & - & - & - & - & 6 \\
\hline & Winter 08 & - & - & - & - & - & - & - & - & - & - & 1 \\
\hline & Spring 08 & - & 4 & - & - & - & - & - & - & - & - & 4 \\
\hline & Summer 08 & - & 2 & - & - & - & - & - & - & - & - & 9 \\
\hline \multirow{4}{*}{$\begin{array}{c}\text { South Scruggs } \\
\text { stream }\end{array}$} & Fall 07 & - & 40 & - & 16 & - & - & - & - & - & - & 157 \\
\hline & Winter 08 & - & 57 & - & 14 & - & - & - & - & - & - & 131 \\
\hline & Spring 08 & - & 52 & - & 37 & - & - & - & - & - & - & 119 \\
\hline & Summer 08 & - & 51 & - & 21 & - & - & - & - & - & - & 103 \\
\hline \multirow{4}{*}{ Marsh spring-pool } & Fall 07 & - & - & - & - & - & - & - & - & - & - & - \\
\hline & Winter 08 & - & - & - & - & - & - & - & - & - & - & - \\
\hline & Spring 08 & - & 1 & - & - & - & - & - & - & - & - & - \\
\hline & Summer 08 & - & - & - & - & - & - & - & - & - & - & - \\
\hline \multirow{4}{*}{ Marsh stream } & Fall 07 & - & 168 & - & - & - & - & - & - & - & - & - \\
\hline & Winter 08 & - & 171 & - & - & - & - & - & - & - & - & - \\
\hline & Spring 08 & - & 141 & - & - & - & - & - & - & - & - & - \\
\hline & Summer 08 & - & 152 & - & - & - & - & - & - & - & - & - \\
\hline \multirow{4}{*}{$\begin{array}{l}\text { North Indian } \\
\text { spring-pool }\end{array}$} & Fall 07 & - & - & - & - & - & - & - & - & - & - & 3 \\
\hline & Winter 08 & - & 1 & - & - & - & - & - & - & - & - & 4 \\
\hline & Spring 08 & - & 1 & - & - & - & - & - & - & - & - & - \\
\hline & Summer 08 & - & 1 & - & - & - & - & - & - & - & - & 3 \\
\hline \multirow{4}{*}{$\begin{array}{l}\text { North Indian } \\
\text { stream }\end{array}$} & Fall 07 & - & 79 & - & 62 & - & - & - & - & - & - & 55 \\
\hline & Winter 08 & - & 34 & - & 17 & - & - & - & - & - & 2 & 34 \\
\hline & Spring 08 & - & 35 & - & 15 & - & - & - & - & - & - & 28 \\
\hline & Summer 08 & - & 32 & - & 4 & - & - & - & - & - & - & 23 \\
\hline \multirow{4}{*}{$\begin{array}{l}\text { South Indian } \\
\text { stream }\end{array}$} & Fall 07 & - & - & - & 22 & - & - & - & - & - & - & 15 \\
\hline & Winter 08 & - & - & - & 2 & - & - & - & - & - & - & 9 \\
\hline & Spring 08 & - & - & - & 3 & - & - & - & - & - & - & 4 \\
\hline & Summer 08 & - & - & - & 3 & - & - & - & - & - & - & 5 \\
\hline \multirow{3}{*}{$\begin{array}{l}\text { School spring- } \\
\text { pool }\end{array}$} & Fall 07 & - & 19 & - & 3 & - & - & - & - & - & - & 4 \\
\hline & Winter 08 & - & 4 & - & - & - & - & - & - & - & - & - \\
\hline & Summer 08 & - & 6 & - & - & - & - & - & - & - & - & - \\
\hline \multirow{2}{*}{ School ponds } & Fall 07 & - & 294 & - & 50 & - & - & - & - & - & - & 21 \\
\hline & Winter 08 & - & 267 & - & 45 & - & - & - & - & - & - & 3 \\
\hline \multirow{3}{*}{ School stream } & Fall 07 & - & 62 & - & 40 & - & - & - & - & - & - & 41 \\
\hline & Winter 08 & - & 116 & - & 24 & - & - & - & - & - & - & 43 \\
\hline & Summer 08 & - & 177 & - & - & - & - & - & - & - & - & - \\
\hline
\end{tabular}


South Scruggs and North Scruggs springs have the longest outflows of the Warm Springs Complex. Warm Springs Amargosa pupfish in South Scruggs Spring was localized in distribution, as demonstrated by kernel density estimation (fig. 5). Most captures were within $150 \mathrm{~m}$ of the springhead and then none until about $800 \mathrm{~m}$ from the springhead (fig. 4). Mosquitofish rarely overlapped Warm Springs Amargosa pupfish and the greatest density of mosquitofish was in the reach with virtually no Warm Springs Amargosa pupfish captures (appendix B: table B1, fig. B1). Crayfish had the widest distribution among the three species, and almost completely overlapped with Warm Springs Amargosa pupfish and mosquitofish. Although the widest distribution of crayfish was in South Scruggs and North Indian Springs, catch per trap and total captures typically were higher for Warm Springs Amargosa pupfish than crayfish in most seasons. We did not weigh captures, but crayfish biomass seemed to exceed that of Warm Springs Amargosa pupfish.

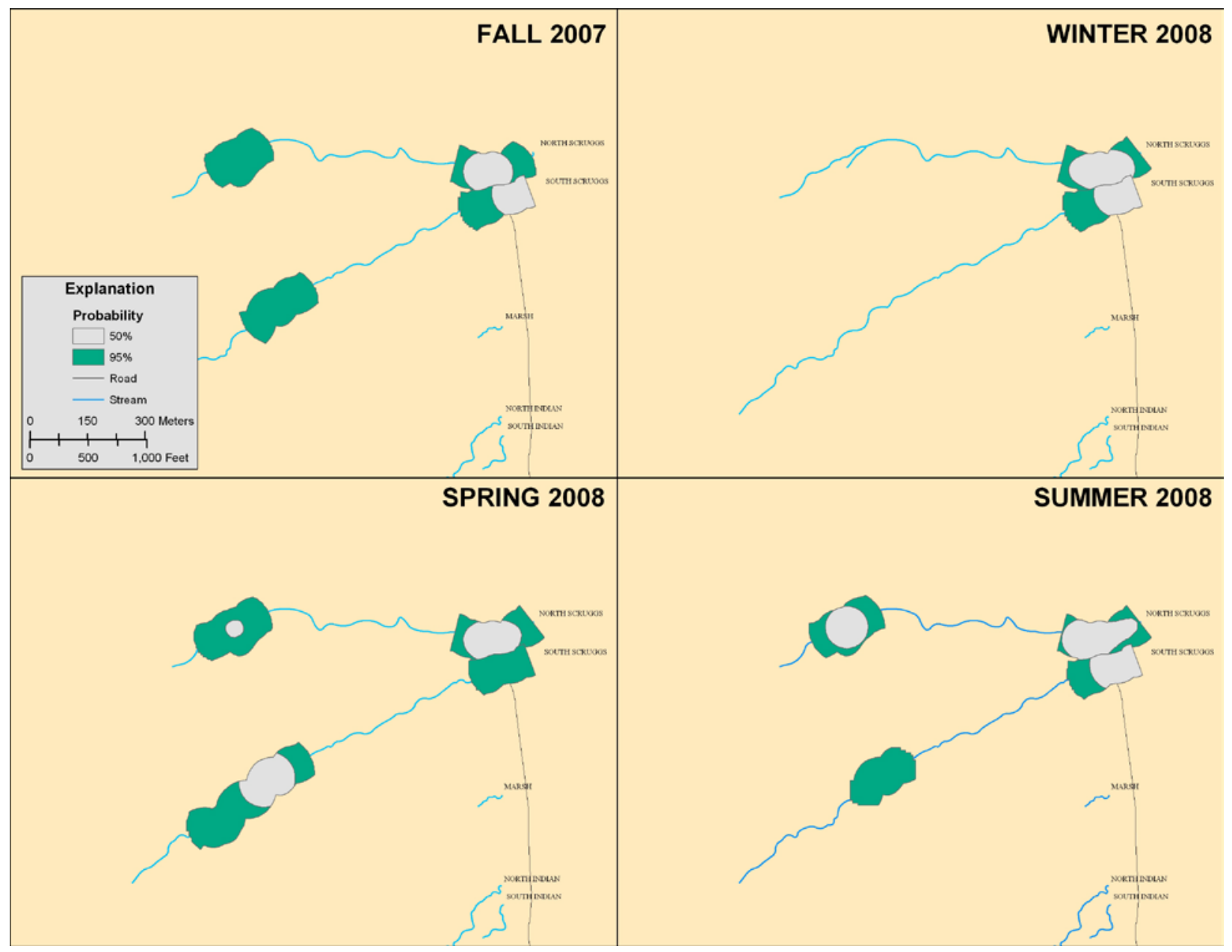

Figure 5. Kemel density estimations (50 and 95 percent probability) of Warm Springs Amargosa pupfish in the Warm Springs Complex, Ash Meadows National Wildlife Refuge, Nevada, fall 2007-summer 2008. 
Total catches and catch per trap generally were high in spring systems without crayfish and mosquitofish. Much of North Scruggs Spring was on private property at the time of our surveys and was not sampled. Marsh Spring had the shortest outflow, did not harbor non-native fish or crayfish, and its lower reach has an artificially formed marsh, which provides substantial Warm Springs Amargosa pupfish habitat (and accounted for most captures).

During this study, School Springs was under restoration, including the eradication of mosquitofish and crayfish and redirection of spring outflow. We sampled the spring system in fall 2007 and winter 2008 prior to restoration and then in summer 2008 after restoration. Crayfish and mosquitofish had been introduced into School Springs soon after 2000 (Shawn Goodchild, U.S. Fish and Wildlife Service, oral commun., 2000). Even after these introductions, School Springs had the highest Warm Springs Amargosa pupfish capture rate. Fish were concentrated in the deep concrete pools that were created to promote Warm Springs Amargosa pupfish in School Springs. There have been concerted efforts to remove all non-native fish from these artificial pools. Our sampling of the restored School Springs confirms that crayfish and mosquitofish were eradicated.

\section{Southern Springs}

Crystal Spring is the largest volume spring in Ash Meadows NWR and its outflow supports the greatest surface area of wetlands, which consists of a spring-pool, outflow, concrete channels, Crystal Reservoir, Horseshoe Reservoir, and Crystal Marsh. Within this extensive system, Ash Meadows Amargosa pupfish captures were greatest in the spring-pool and streams (fig. 6). Relatively few captures were from reservoir and marsh habitats, especially Crystal Reservoir and Crystal Marsh. These areas were occupied by largemouth bass and green sunfish. Where centrarchids occurred, Ash Meadows Amargosa pupfish, crayfish, and mosquitofish were scarce. Ash Meadows Amargosa pupfish was the predominant species captured in Crystal spring-pool and outflow, followed by crayfish, mosquitofish, and sailfin molly (table 3). Like Ash Meadows Amargosa pupfish, sailfin molly and crayfish were captured with high frequently in the upper $800 \mathrm{~m}$ of the outflow, and with high frequency in Horseshoe Marsh (appendix C: figs. C2 and C5).

Kings Pool spring-pool, like the other Ash Meadows NWR warm water spring-pools, had substantially greater Ash Meadows Amargosa pupfish captures per trap compared to its outflow. Highest spring-pool capture was in fall 2007 when 838 Ash Meadows Amargosa pupfish were captured with a success rate of 139.7 captures per trap (table 3, appendix C: table C1). Ash Meadows Amargosa pupfish were observed along the entire course of Kings Pool outflow; the greatest concentration of Ash Meadows Amargosa pupfish was within $500 \mathrm{~m}$ of the spring-pool (fig. 6). Greatest concentrations of crayfish were observed $500 \mathrm{~m}$ downstream of the spring-pool (appendix C: fig. C5). Although somewhat localized in distribution, more Ash Meadows Amargosa pupfish were captured than non-native fish (table 3). 


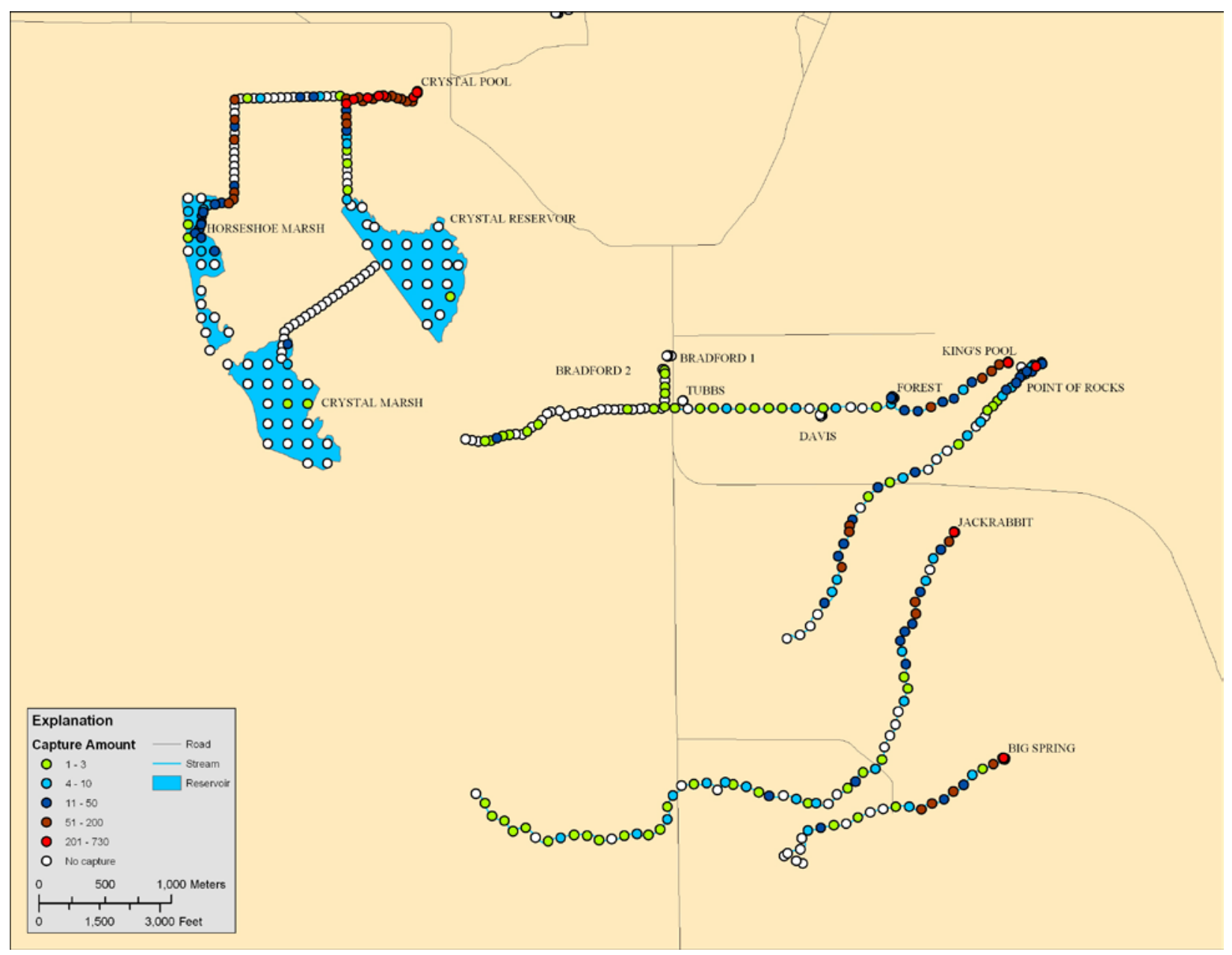

Figure 6. Relative abundance and distribution of Ash Meadows Amargosa pupfish in the southern springs, Ash Meadows National Wildlife Refuge, Nevada, fall 2007-summer 2008. 
Table 3. Seasonal catches of fishes, bullfrogs, and crayfish at the southern springs of the Ash Meadows National Wildlife Refuge, Nevada, fall 2007-summer 2008.

[Spring locations are shown in figures 1 and 2. CYMI, Ash Meadows Amargosa pupfish; CYPE, Warm Springs Amargosa pupfish; RHON, Ash Meadows speckled dace; GAAF, Mosquitofish; POLA, Sailfin Molly; LECY, Green Sunfish; MISA, Largemouth Bass; ARNI, Convict Cichlid; AMME, Black Bullhead; LICA, Bull Frog; PRCL, Red Swamp Crayfish]

\begin{tabular}{|c|c|c|c|c|c|c|c|c|c|c|c|c|}
\hline System & Season & CYMI & CYPE & RHON & GAAF & POLA & LECY & MISA & ARNI & AMME & LICA & PRCL \\
\hline \multirow{4}{*}{$\begin{array}{l}\text { Crystal spring- } \\
\text { pool }\end{array}$} & Fall 07 & 319 & - & - & 66 & 1 & - & - & - & - & - & 70 \\
\hline & Winter 08 & 594 & - & - & 31 & - & - & - & - & - & - & 68 \\
\hline & Spring 08 & 564 & - & - & 21 & 2 & - & - & - & - & - & 24 \\
\hline & Summer 08 & 554 & - & - & 27 & - & - & - & - & - & - & 34 \\
\hline \multirow{4}{*}{ Crystal stream } & Fall 07 & 1,423 & - & - & 339 & 105 & - & - & - & - & - & 609 \\
\hline & Winter 08 & 1,099 & - & - & 318 & 17 & - & - & - & - & - & 639 \\
\hline & Spring 08 & 646 & - & - & 190 & 11 & - & - & - & - & - & 402 \\
\hline & Summer 08 & 958 & - & - & 242 & 3 & - & - & - & - & - & 605 \\
\hline \multirow{4}{*}{$\begin{array}{l}\text { Crystal } \\
\text { reservoir }\end{array}$} & Fall 07 & 1 & - & - & - & - & 386 & 3 & - & - & - & - \\
\hline & Winter 08 & - & - & - & - & - & 4 & - & - & - & - & - \\
\hline & Spring 08 & 5 & - & - & - & - & 9 & 5 & - & - & - & - \\
\hline & Summer 08 & - & - & - & - & - & 77 & - & - & - & - & - \\
\hline \multirow{2}{*}{ Crystal stream } & Fall 07 & - & - & - & 1 & - & 10 & - & - & - & - & 1 \\
\hline & Winter 08 & - & - & - & - & - & 5 & - & - & - & - & 1 \\
\hline \multirow{4}{*}{ Crystal marsh } & Fall 07 & 24 & - & - & 66 & - & 26 & - & - & - & - & 3 \\
\hline & Winter 08 & 14 & - & - & 5 & - & 4 & - & - & - & - & 1 \\
\hline & Spring 08 & 6 & - & - & - & - & 27 & 16 & - & - & - & 3 \\
\hline & Summer 08 & 4 & - & - & 4 & - & 41 & 1 & - & - & - & - \\
\hline \multirow{4}{*}{$\begin{array}{l}\text { Horseshoe } \\
\text { marsh }\end{array}$} & Fall 07 & 17 & - & - & 97 & 54 & - & - & - & - & 1 & 210 \\
\hline & Winter 08 & 27 & - & - & 18 & 34 & 1 & - & - & - & 11 & 81 \\
\hline & Spring 08 & 72 & - & - & 16 & 19 & 5 & - & - & - & 2 & 174 \\
\hline & Summer 08 & 12 & - & - & 437 & 44 & - & - & - & - & 37 & 162 \\
\hline \multirow{4}{*}{$\begin{array}{l}\text { Kings Pool } \\
\text { spring-pool }\end{array}$} & Fall 07 & 838 & - & - & 4 & - & - & - & - & - & - & - \\
\hline & Winter 08 & 531 & - & - & 16 & - & - & - & - & - & - & - \\
\hline & Spring 08 & 653 & - & - & 1 & - & - & - & - & - & - & - \\
\hline & Summer 08 & 701 & - & - & 2 & - & - & - & - & - & - & - \\
\hline \multirow{4}{*}{$\begin{array}{l}\text { Kings Pool } \\
\text { stream }\end{array}$} & Fall 07 & 179 & - & 1 & 57 & 29 & - & - & - & - & - & 80 \\
\hline & Winter 08 & 106 & - & - & 35 & 12 & - & - & - & - & - & 47 \\
\hline & Spring 08 & 170 & - & - & 29 & 29 & - & - & - & - & - & 78 \\
\hline & Summer 08 & 157 & - & - & 74 & 41 & - & - & - & - & - & 104 \\
\hline \multirow{4}{*}{$\begin{array}{l}\text { Point of Rocks } \\
\text { stream }\end{array}$} & Fall 07 & 237 & - & - & 29 & 5 & - & - & - & - & - & 196 \\
\hline & Winter 08 & 179 & - & 5 & 91 & 7 & - & - & - & - & 1 & 91 \\
\hline & Spring 08 & 227 & - & 2 & 49 & 6 & - & - & - & - & - & 148 \\
\hline & Summer 08 & 234 & - & - & 119 & 3 & - & - & - & - & - & 198 \\
\hline \multirow{4}{*}{$\begin{array}{l}\text { Bradford } 1 \\
\text { spring-pool }\end{array}$} & Fall 07 & - & - & 615 & 125 & - & - & - & - & - & - & 37 \\
\hline & Winter 08 & - & - & 694 & 447 & - & - & - & - & - & - & 15 \\
\hline & Spring 08 & - & - & 383 & 51 & - & - & - & - & - & - & 29 \\
\hline & Summer 08 & - & - & 265 & 334 & - & - & - & - & - & - & 29 \\
\hline \multirow{4}{*}{$\begin{array}{l}\text { Bradford } 1 \\
\text { stream }\end{array}$} & Fall 07 & - & - & 80 & 93 & - & - & - & - & - & - & 30 \\
\hline & Winter 08 & - & - & 77 & 78 & - & - & - & - & - & - & 18 \\
\hline & Spring 08 & - & - & - & 261 & - & - & - & - & - & - & 13 \\
\hline & Summer 08 & - & - & 33 & 692 & - & - & - & - & - & - & 26 \\
\hline \multirow{4}{*}{$\begin{array}{l}\text { Bradford } 2 \\
\text { spring-pool }\end{array}$} & Fall 07 & - & - & 31 & 358 & - & - & - & - & - & - & 153 \\
\hline & Winter 08 & - & - & 9 & 390 & 141 & - & - & - & - & - & 52 \\
\hline & Spring 08 & - & - & - & - & - & - & - & - & - & 1 & 95 \\
\hline & Summer 08 & - & - & 2 & 31 & - & - & - & - & - & - & 40 \\
\hline
\end{tabular}


Table 3. Seasonal catches of fishes, bullfrogs, and crayfish at the southern springs of the Ash Meadows National Wildlife Refuge, Nevada, fall 2007-summer 2008.-Continued.

\begin{tabular}{|c|c|c|c|c|c|c|c|c|c|c|c|c|}
\hline System & Season & CYMI & CYPE & RHON & GAAF & POLA & LECY & MISA & ARNI & AMME & LICA & PRCL \\
\hline \multirow{4}{*}{ Bradford 2 stream } & Fall 07 & 15 & - & 95 & 163 & 22 & - & - & - & - & 1 & 141 \\
\hline & Winter 08 & 3 & - & 94 & 130 & 14 & - & - & - & - & 1 & 100 \\
\hline & Spring 08 & 14 & - & 79 & 88 & 1 & - & - & - & - & 2 & 85 \\
\hline & Summer 08 & 5 & - & 78 & 123 & 4 & - & - & - & - & - & 120 \\
\hline \multirow{4}{*}{ Forest spring-pool } & Fall 07 & 1 & - & 5 & 462 & 9 & - & - & - & - & - & 140 \\
\hline & Winter 08 & 2 & - & 7 & 397 & 15 & - & - & - & - & - & 45 \\
\hline & Spring 08 & 55 & - & 7 & 287 & 1 & - & - & - & - & - & 152 \\
\hline & Summer 08 & 42 & - & 1 & 415 & 4 & - & - & - & - & - & 140 \\
\hline \multirow{4}{*}{ Tubbs spring-pool } & Fall 07 & - & - & - & - & - & - & - & - & - & 4 & 6 \\
\hline & Winter 08 & - & - & - & - & - & - & - & - & - & - & 16 \\
\hline & Spring 08 & - & - & - & - & - & - & - & - & - & 2 & 49 \\
\hline & Summer 08 & - & - & - & - & - & - & - & - & - & 8 & 51 \\
\hline \multirow{4}{*}{ Davis spring-pool } & Fall 07 & - & - & - & 119 & - & - & - & - & 8 & - & 5 \\
\hline & Winter 08 & - & - & - & 1 & - & - & - & - & - & - & 4 \\
\hline & Spring 08 & - & - & - & 359 & - & - & - & - & 2 & - & 19 \\
\hline & Summer 08 & - & - & - & - & - & - & - & - & - & - & 11 \\
\hline \multirow{4}{*}{$\begin{array}{l}\text { Jackrabbit spring- } \\
\text { pool }\end{array}$} & Fall 07 & 786 & - & 19 & 55 & 69 & - & - & - & - & - & 25 \\
\hline & Winter 08 & 726 & - & 21 & 52 & 35 & - & - & - & - & - & 24 \\
\hline & Spring 08 & 761 & - & 31 & 1 & 75 & - & - & - & - & - & 19 \\
\hline & Summer 08 & 512 & - & 23 & 28 & 32 & - & - & - & - & - & 43 \\
\hline \multirow{4}{*}{ Jackrabbit stream } & Fall 07 & 79 & - & 510 & 121 & 36 & - & - & - & - & - & 291 \\
\hline & Winter 08 & 169 & - & 645 & 56 & 3 & - & - & - & - & - & 295 \\
\hline & Spring 08 & 175 & - & 652 & 91 & 8 & - & - & - & - & 1 & 288 \\
\hline & Summer 08 & 147 & - & 607 & 333 & 11 & - & - & - & - & - & 461 \\
\hline \multirow{4}{*}{$\begin{array}{l}\text { Big Spring spring- } \\
\text { pool }\end{array}$} & Fall 07 & 157 & - & - & 2 & 40 & - & - & - & - & - & - \\
\hline & Winter 08 & 416 & - & - & 13 & 9 & - & - & - & - & 15 & 1 \\
\hline & Spring 08 & 107 & - & - & 5 & 5 & - & 10 & - & - & - & - \\
\hline & Summer 08 & 213 & - & - & 128 & 32 & - & - & - & - & 8 & 7 \\
\hline \multirow{4}{*}{ Big Spring stream } & Fall 07 & 253 & - & - & 14 & 42 & - & - & - & - & 6 & 117 \\
\hline & Winter 08 & 206 & - & - & 36 & 8 & - & - & - & - & 14 & 92 \\
\hline & Spring 08 & 117 & - & - & 30 & 18 & - & - & - & - & - & 120 \\
\hline & Summer 08 & 61 & - & - & 109 & 44 & - & - & - & - & 2 & 54 \\
\hline
\end{tabular}

In the Point-of-Rocks Spring system, Ash Meadows Amargosa pupfish capture was greatest in the upper 1,000 m. As with Kings Pool and Crystal Springs, Ash Meadows Amargosa pupfish was captured with greater frequency than crayfish, mosquitofish, or sailfin molly. Speckled dace was captured only in winter $2008(n=5)$ and spring $2008(n=2)$ in the Point-ofRocks Spring system.

Bradford 1 and Bradford 2 Springs are relatively cool springs harboring Ash Meadows speckled dace and a few Ash Meadows Amargosa pupfish. Speckled dace capture was particularly high in Bradford 1 Spring ranging from 298 (summer 2008) to 771 (winter 2008) (table 3). Speckled dace was the predominant capture for all seasons except summer, when mosquitofish predominated. Crayfish captures were consistently the lowest for each season. No Ash Meadows Amargosa pupfish was found in Bradford 1 Spring but was found in Bradford 2 Spring (fig. 6). 
In Forest Spring, seasonal Ash Meadows Amargosa pupfish captures ranged from 1 (fall 2007) to 55 (summer 2008) (fig. 6) and seasonal Ash Meadows speckled dace captures ranged from 1 (summer 2008) to 7 (winter 2008) (fig. 7). Mosquitofish was captured with the greatest frequency followed by crayfish (appendix C: table C1, figs. C1 and C5). Sailfin molly captures were low (appendix C: fig. C2).

During this study, no native fish were captured in Tubbs and Davis Springs. Davis Spring supported mosquitofish, black bullhead, and crayfish in fall 2007, winter 2008, and spring 2008. Davis Spring was chemically treated in 2008 with rotenone to eliminate black bullhead, and only crayfish were captured in summer 2008 (appendix C: table C1, figs. C1 and C5).

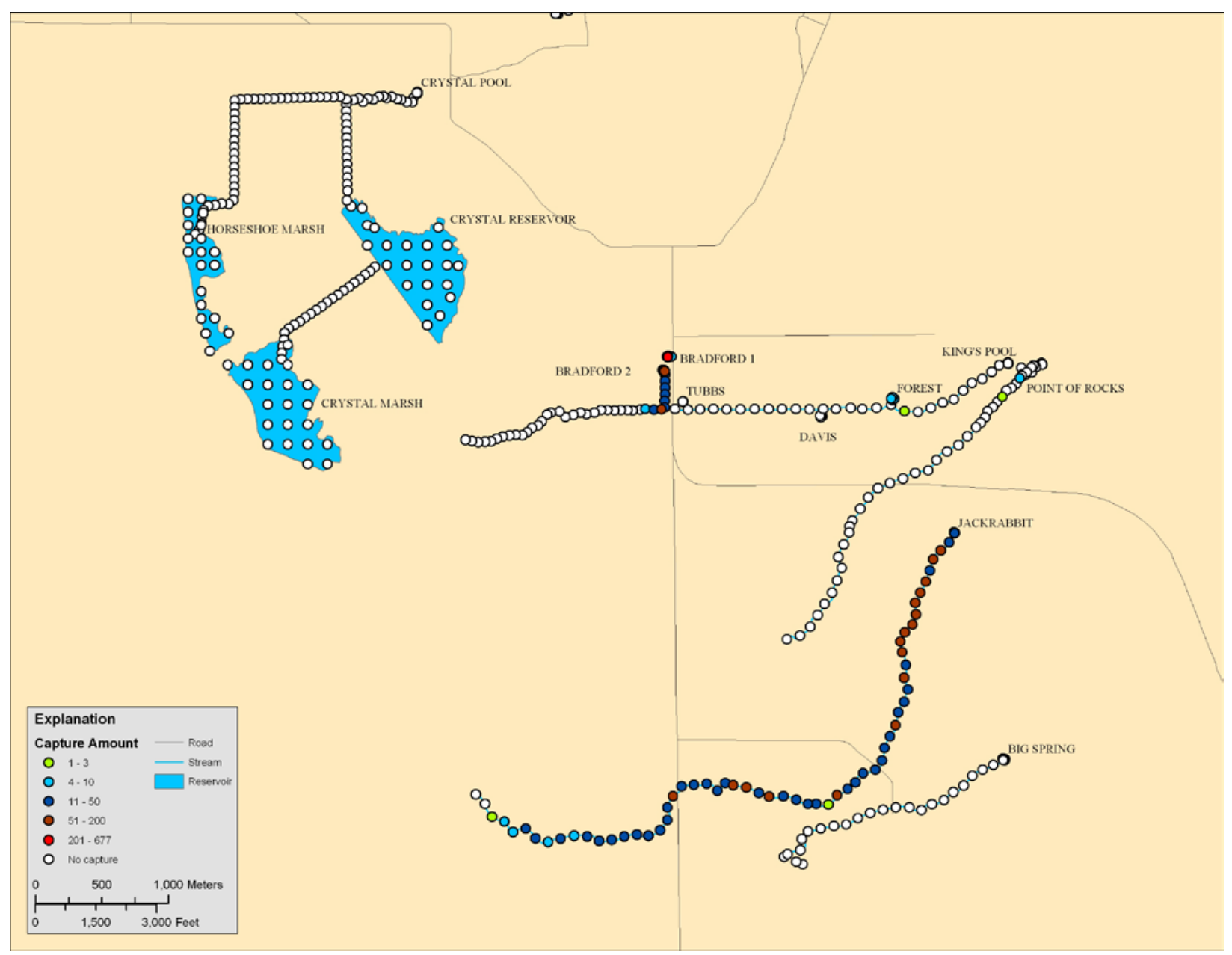

Figure 7. Relative abundance and distribution of Ash Meadows speckled dace in the southern springs, Ash Meadows National Wildlife Refuge, Nevada, fall 2007-summer 2008. 
In Jackrabbit Spring, Ash Meadows Amargosa pupfish was the predominant species captured in the spring-pool with catches ranging from 786 (fall 2007) to 512 (summer 2008) although Ash Meadows speckled dace was the predominant species captured in the outflow with catches ranging from 659 (spring 2008) to 510 (fall 2007). During the four seasons of trapping in Jackrabbit Spring, speckled dace had been captured at every station except the lowest two (fig. 7). There were trends in Ash Meadows Amargosa pupfish and speckled dace seasonal outflow distribution that lend themselves well to kernel density estimation. The highest density of Ash Meadows Amargosa pupfish in Jackrabbit Spring outflow was in the upper 1,000 m, and particularly in the $500 \mathrm{~m}$ restoration site (fig. 8). About 8 percent of the total outflow supported 40-60 percent of the Ash Meadows Amargosa pupfish through the four seasons. Speckled dace also congregated at the restoration site especially in fall 2007 and winter 2008 with 33 percent of the captures there. In spring 2008, speckled dace was more widely dispersed and only about 14 percent of the captures were from the restoration area (fig. 9).

The capture frequency of Ash Meadows Amargosa pupfish was highest in Big Springpool in all seasons; however, few Ash Meadows Amargosa pupfish were found in the outflow and western mosquitofish was the predominant species. The greatest concentration of Ash Meadows Amargosa pupfish was in the upper $800 \mathrm{~m}$ of the stream. No speckled dace were captured in Big Spring. Crayfish was abundant in the outflow, but relatively few crayfish were captured from the spring-pool. 


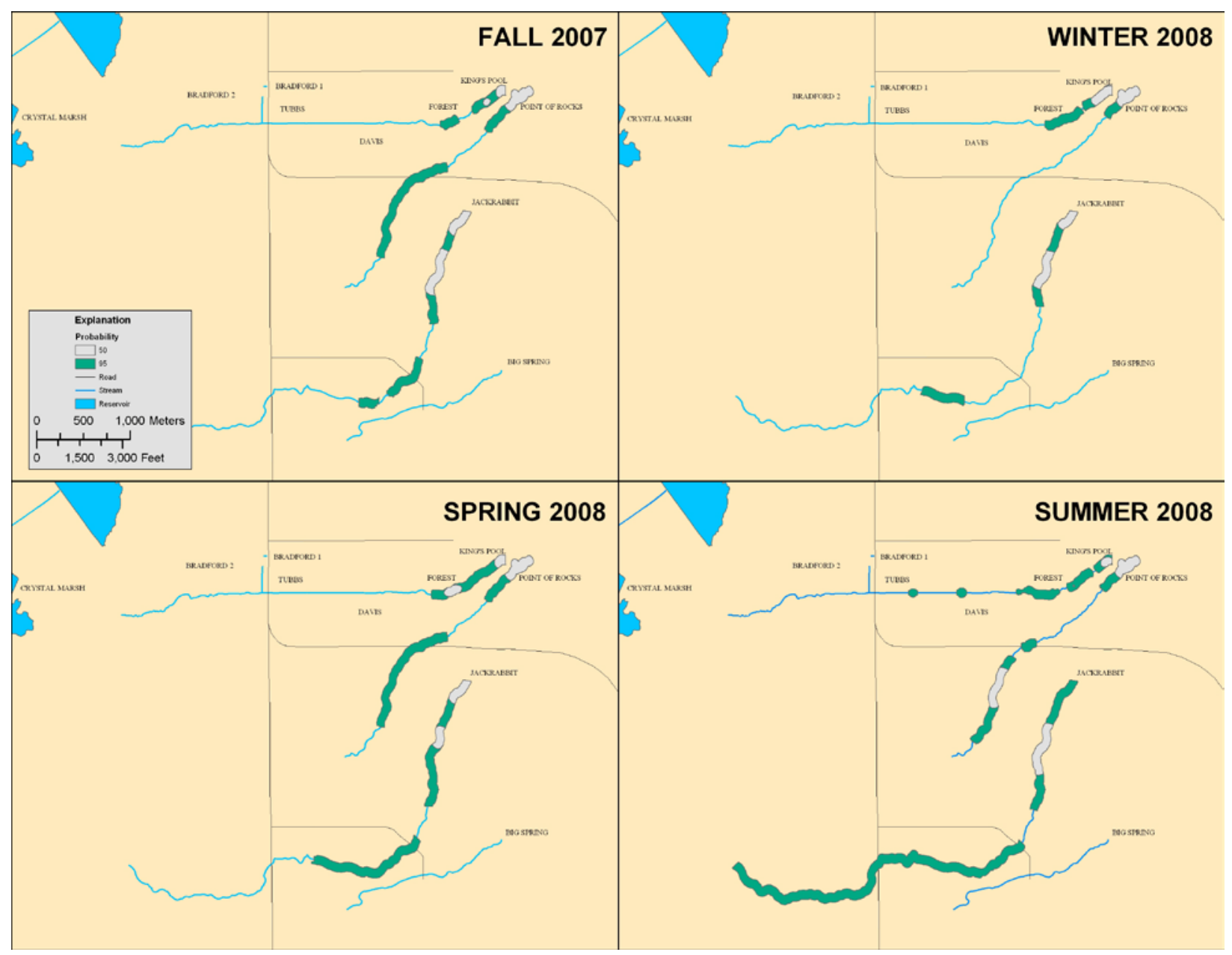

Figure 8. Kemel density estimations (50 and 95 percent probability) of Ash Meadows Amargosa pupfish in King's Pool, Point of Rocks, and Jackrabbit Springs, Ash Meadows National Wildlife Refuge, Nevada, fall 2007-summer 2008. 


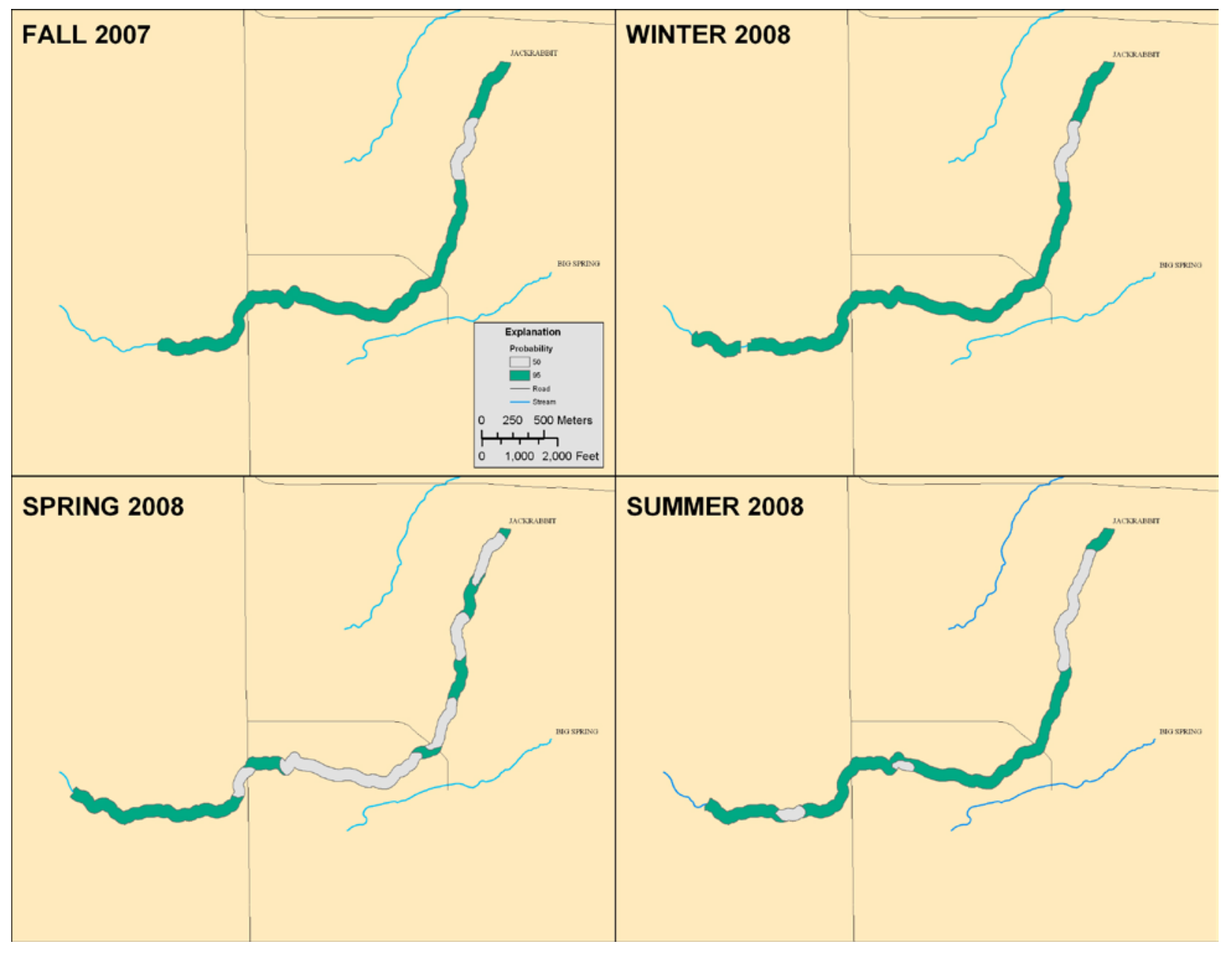

Figure 9. Kemel density estimations (50 and 95 percent probability) of Ash Meadows speckled dace in Jackrabbit Spring, Ash Meadows National Wildlife Refuge, Nevada, fall 2007-summer 2008. 


\section{Discussion}

Although pupfish and speckled dace can survive and thrive in a wide variety of habitats (Moyle, 2002), there are habitat types that promote invasive species at the expense of pupfish and speckled dace (Scoppettone and others, 2005). The formation of Lower Crystal Marsh was an early Crystal Spring rehabilitation project to expand refuge wetlands and increase Ash Meadows Amargosa pupfish habitat (Threloff, 1991). The 42 ha wetland was completed in the early 1990s and within 2 years Ash Meadows Amargosa pupfish numbered more than 100,000 (Threloff, 1990). Thereafter, largemouth bass and green sunfish invaded the marsh, and our low Ash Meadows Amargosa pupfish captures suggest high predation. Lacustrine habitat not only serves as a haven for these predators, but can promote sailfin molly and mosquitofish as well. The formation of a headwater marsh immediately downstream of Kings Pool that was caused by a breach in a decaying and eroding outflow channel promoted sailfin molly, which was the predominant species there (Scoppettone and others, 2005). Formation of the Kings Pool headwater marsh began in 1989, and was capturing almost all the outflow water by 1994. In 1997, an outflow channel was excavated through the headwater marsh and trenched to an old irrigation ditch flowing west to into Forest and Bradford 2 Springs outflow. This restored reach transformed a sailfin molly-predominated marsh to an Ash Meadows Amargosa pupfishpredominated outflow. Our survey indicated that Ash Meadows Amargosa pupfish remained the predominant species in the outflow and in the spring-pool. Sailfin molly and mosquitofish were the predominant species in Kings Pool spring-pool prior to the restoration of the outflow, but Ash Meadows Amargosa pupfish predominated following restoration.

Native fish abundance in Bradford 1 and Bradford 2 Springs seem to be linked to status change of non-native species. Refuge personnel have been continually and systematically removing crayfish from Bradford 1 Spring, and these data suggest this measure has been effective. Seasonal numbers of Ash Meadows speckled dace reported from Bradford 1 Spring were much greater compared to an earlier estimate. In winter 2008, almost 700 individuals were captured, although in March 2002 only $63 \pm 4$ were estimated (Leavy and others, 2004). Captures from Bradford 2 Spring were not as great as Bradford 1 Spring, but capture numbers and size structure suggest a reproductive population. In 1990, the Ash Meadows Amargosa pupfish population had been extirpated after a largemouth bass invasion. Bass has since been eliminated from the system and Ash Meadows Amargosa pupfish has been able to recolonize the spring after extensive crayfish removal (Leavy and others, 2004). One green sunfish was found in the system in 2001, and it was removed before substantial loss of Ash Meadows speckled dace (Leavy and others, 2004). Previous surveys of the Bradford Springs are difficult to compare with these results because the surveys were somewhat subjective (Hardy, 1979, Threloff, 1990) and report three Bradford springs while this study could only account for two springs. When those early surveys were conducted, Kings Pool outflow connected Bradford 1 and Bradford 2 Springs immediately adjacent to their respective spring-pools; whether this flow was perceived as a third spring is not clear. 
Tubbs, Big, and Jackrabbit Springs reportedly harbored Ash Meadows speckled dace when the Ash Meadows NWR was established (Sada, 1990). The Tubbs Spring population of dace was estimated to be 35 fish, although no fish were captured by Threloff (1990) or during this study. In Big Spring, Ash Meadows speckled dace and Ash Meadows Amargosa pupfish were reported as common when the system was sampled in summer 1990 (Scoppettone and others, 1995), but by the late 1990s, the largemouth bass invasion into the spring became a persistent problem even after eradication efforts. At the end of this first year survey, largemouth bass were thought to be few or absent (Darrick Wessienfluh, Ash Meadows National Wildlife Refuge, oral commun., 2008). No Ash Meadows speckled dace were captured, suggesting that these fish were rare or have been extirpated from the Big Spring system. Ash Meadows Amargosa pupfish was common.

When the Ash Meadows NWR was established, Jackrabbit Spring was reported to harbor Ash Meadows speckled dace and Ash Meadows Amargosa pupfish (Williams and Sada, 1985) and it continues to do so. The Jackrabbit Spring fish community has not changed in more than 25 years. However, alterations made to the Jackrabbit environment, both floristic and hydraulic, would be expected to influence the abundance and distribution of native fishes. A fire along Jackrabbit outflow in August 2005 led to stream rehabilitation to protect the native species. Removal of tamarisk along the riparian corridor improved habitat conditions for native fishes (Kennedy and others, 2006). Marsh-like habitat along the course (which promotes nonnative fishes and crayfish) was transformed to a flowing stream, which was demonstrated to promote native fishes (Scoppettone and others, 2005). The most obvious change in native fish complement was a substantial increase in catch rate of speckled dace compared to the catch rate reported by Scoppettone and others (1995). The greatest concentration of native fishes in the stream was along the $500 \mathrm{~m}$ of restored habitat indicating, that at least in the early stage, the restoration was a success. Restoration habitat was transformed from marsh-like to flowing stream.

Forest and Point-of-Rocks Springs had relatively recent introductions of Ash Meadows speckled dace. Forest Spring was reported by Miller (1948) to have harbored Ash Meadows speckled dace, but Sada (1990) did not report Ash Meadows speckled dace when Ash Meadows NWR was established, and few Ash Meadows Amargosa pupfish were observed (Threloff, 1990). Forest Spring was one of several springs on the southern side of the Ash Meadows NWR to have been invaded by largemouth bass (chemically removed in 1998). We suspect that the few Ash Meadows Amargosa pupfish we captured from Forest Spring had re-colonized from Kings Pool Spring and the few Ash Meadows speckled dace captured were from those that had been introduced or had emigrated from Bradford 2 Spring.

The upper section of the Point-of-Rocks Spring system was restored in 2001 and converted from marsh-like to flowing stream. Although no baseline information on an Ash Meadows Amargosa pupfish population is available, we presume this restoration had a positive influence on Ash Meadows Amargosa pupfish. Outflow water temperature for these springs appeared suitable for Ash Meadows speckled dace, which were introduced in 2002 (Shawn Goodchild, U.S. Fish and Wildlife Service, oral commun.,2002). Few Ash Meadows speckled dace were captured, suggesting dace may be having difficulty establishing in a system with climax populations of crayfish and poeciliids. 
Some changes have been made to aquatic habitats at the northern springs that would affect native fish communities, but not of the same magnitude as in the southern springs. The northern springs also have only one native fish species, Ash Meadows Amargosa pupfish. Of the northern springs, Fairbanks Spring probably has had the most fish community-altering changes since the establishment of the Ash Meadows NWR. Among the most outstanding changes were the 1998 fire along the course of the outflow, formation of headwater marsh, and the 2001 invasion of convict cichlids. The effect the fire had on the Ash Meadows Amargosa pupfish population is unknown, but formation of headwater marsh presumably had a negative effect (Scoppettone and others, 2005). Convict cichlids greatly suppressed the Ash Meadows Amargosa pupfish population. The planned restoration of Fairbanks Spring has the potential to positively influence the Ash Meadows Amargosa pupfish population.

Our captures of Ash Meadows Amargosa pupfish in the Rogers spring-pool were more than four times the estimates made in the early 1990s (Threloff, 1990; Scoppettone and others, 1995). No alteration to Rogers Spring has been documented and the reason for this increase is unknown.

The only change to Longstreet Spring has been directing the outflow to Petersen Reservoir intermittently since 1985, either through a pipe on its southwestern side of the springpool or an open ditch to the west. At the time of our survey, water was flowing down the open ditch and Ash Meadows Amargosa pupfish density in the outflow was among the lowest of the larger Ash Meadows springs. It is suspected that this low density is due, at least in part, to the greatly enlarged spring-pools that cool water from springs. Water temperature in the outflow is less than the Amargosa pupfish reproductive temperature minimum requirement of $26^{\circ} \mathrm{C}$ (Soltz and Naiman, 1978).

The primary impact to Warm Springs Amargosa pupfish habitat since the establishment of the Ash Meadows NWR has been the spread of crayfish and mosquitofish. When these two species first invaded the Warm Springs Complex is not clear, but these two species were not reported to be in Indian Spring in the early 1970s (Miller and Deacon, 1973). This is important because South Indian Spring is the first spring these species were documented to invade (Sada, 1990). Sometime after the early 1990s, crayfish and mosquitofish invaded North Indian Spring, and in the early 2000s, these fish were noted to have invaded School Springs, and more recently South Scruggs Spring. Warm Springs Amargosa pupfish no longer inhabit South Indian Spring and have a patchy distribution in South Scruggs Spring, which gives cause for concern that nonnative species may continue to spread and cause a decline of Warm Springs Amargosa pupfish.

It has been previously demonstrated that non-native species have suppressed and replaced Ash Meadows' native fish populations (Scoppettone and others, 2005; Kennedy and others, 2006); this study adds further insight into the extent of non-native species impacts. For example, the Ash Meadows NWR chemical removal of non-native convict cichlid from Fairbanks Spring and mechanical removal of crayfish from Bradford 1 Spring were followed by substantial increases in native fish populations in those respective systems. Warm Springs Amargosa pupfish in North Indian Spring has been completely replaced by crayfish and mosquitofish. Additionally, Ash Meadows native fishes are now localized throughout their historical range, and non-natives species are presumably the cause.

Further data collection and analysis will be aimed at determining environmental factors that promote native fishes instead of crayfish, mosquitofish, and sailfin molly so that these environmental attributes can be integrated into restoration design and implementation. This study provides the baseline data for determining the success of future habitat design and modification. 


\section{Acknowledgments}

Thanks to Ash Meadows National Wildlife Refuge for funding the project, and assisting us with lodging. Antonio Salgado (USGS), Mark Fabes (USGS), Sean Shea (USGS), Eric Miskow (Nevada Natural Heritage Program), and Shawn Goodchild (USFWS) assisted with fieldwork. Thanks to Tom Strekal (BIA Biologist, retired) for critical review.

\section{References Cited}

Baugh, T., Williams, J.E., Buck, D.A., and Deacon, J.E., 1986, New distributional records for Cyprinodon nevadensis mionectes, and endangered pupfish from Ash Meadows, Nevada: The Southwest Naturalist, v. 31, p. 544-546.

Deacon, J.E., Hubbs, C., and Zahuranec, B.J., 1964, Some effects of introduced fishes on the native fish fauna of southern Nevada: Copeia, p. 384-388.

Deacon, J.E., and Williams, C.D., 1991, Ash Meadows and the legacy of the Devils Hole pupfish, in Minckley, W.L., and Deacon, J.E. (eds.), Battle against extinction-Native fish management in the American West: Tucson, The University of Arizona Press, p. 69-87.

Dudley, W.W., Jr., and Larson, J.D., 1976, Effect of irrigation pumping on desert pupfish habitats in Ash Meadows, Nye County, Nevada: U.S. Geological Survey Professional Paper 927, 153 p.

Hardy, T., 1979, The inter-basin area report: Las Vegas, University of Nevada, U.S. Fish and Wild Service Agreement No. 14-16-001-6319-FS Amendment \#4.

Hooge, P.N., and Eichenlaub, B., 1997, Animal movement extension to Arcview, ver. 1.1: Alaska Biological Science Center, U.S. Geological Survey, Anchorage, AK.

Hubbs, C., and Deacon, J.E., 1964, Additional introductions of tropical fishes into southern Nevada: Southwest Naturalist, v. 9, p. 249-251.

Gourley, C.R., and Ammon, E.M., 1997, Restoration of the Kings Spring drainage in Ash Meadows, Nevada: The Nature Conservancy of Nevada.

Kennedy, T.A., Finlay, J.C., and Hobbie, S.E., 2006, Eradication of invasive Tamarix ramosissima along a desert stream increase native fish density: Ecological Applications, v. 15, p. 2072-2083.

La Rivers, I., 1962, Fishes and fisheries of Nevada: Nevada State Fish and Game Commission, $782 \mathrm{p}$.

Leavy, T., McShane, R.R., Swaim, K.M., and Scoppettone G.G., 2004, Status of Ash Meadows speckled dace in Bradford Springs: Final report to the Las Vegas Office of the U.S. Fish and Wildlife Service, 12 p.

Miller, R.R., 1948, The cyprinodon fishes of Death Valley system of eastern California and southeastern Nevada: Miscellaneous Publications of the Museum of Zoology, University of Michigan, v. 529, 55 p.

Miller, R.R., and Deacon, J.E., 1973, New localities for the rare Warm Springs pupfish, Cyprinodon nevadensis pectoralis, from Ash Meadows, Nevada: Copeia, p. 137-140.

Moyle, P.B., 2002, Inland fishes of California: Berkeley, University of California Press.

Pister, E.P., 1974, Desert fishes and their habitats: Transactions of the American Fisheries Society, v. 103, p. 531-540.

Sada, D.W., 1990, Recovery plan for the endangered and threatened species of Ash Meadows, Nevada: Reno, Nev., U.S. Fish and Wildlife Service. 
Scoppettone, G.G., Rissler, P.H., Byers, S., Shea, S., Nielsen, B., and Sjoberg, J., 1995, Information on the status and ecology of Ash Meadows Fishes and Ambrysus: National Biological Service, Reno Field Station, 111 p.

Scoppettone, G.G., Rissler, P.H., Gourley, C., and Martinez, C., 2005, Habitat restoration as a means of controlling non-native fish in a Mojave Desert Oasis: Restoration Ecology, v. 13, p. 247-256.

Soltz, K.L., and Naiman, R.J., 1978, The natural history of native fishes in the Death Valley system: Natural History Museum of Los Angeles County, California, Science Series, v. 30, $76 \mathrm{p}$.

Threloff, D., 1990, The distribution and abundance of the fishes of Ash Meadows-A preliminary inventory: Ash Meadow National Wildlife Refuge, U.S. Fish and Wildlife Service. Threloff, D., 1991, Threatened And Endangered Species Use of a Restored Marsh on the Ash Meadows National Wildlife Refuge: Ash Meadow National Wildlife Refuge, U.S. Fish and Wildlife Service.

U.S. Department of the Interior, 1973, Threatened wildlife of the United States: U.S. Bureau of Sport Fisheries and Wildlife Resources Publication 114, 289 p.

U.S. Environmental Protection Agency, 2010, National Environmental Policy Act (NEPA). Williams, B.K., Nichols, J.D., and Conroy, M.J., 2001, Analysis and management of animal populations (modeling, estimation, and decision making): San Francisco, Calif., Academic Press.

Williams, J.E., and Deacon, J.E., 1986, Sub-specific identity of the Amargosa pupfish, Cyprinodon nevadensis, from Crystal Spring, Ash Meadows, Nevada: Great Basin Naturalist, v. 46, p. 220-223.

Williams, J.E., and Sada, D.W., 1985, Status of two endangered fishes, Cyprinodon nevadensis mionectes and Rhinichthys osculus nevadensis, from two springs in Ash Meadows, Nevada: Southwestern Naturalist, v. 30, p. 475-484.

Worton, B.J., 1987, A review of models of home range for animal movement: Ecological Modeling, v. 38, p. 277-298. 
Appendix A. Seasonal Distributions of Fishes and Crayfish at Ash Meadows National Wildlife Refuge, Nevada 


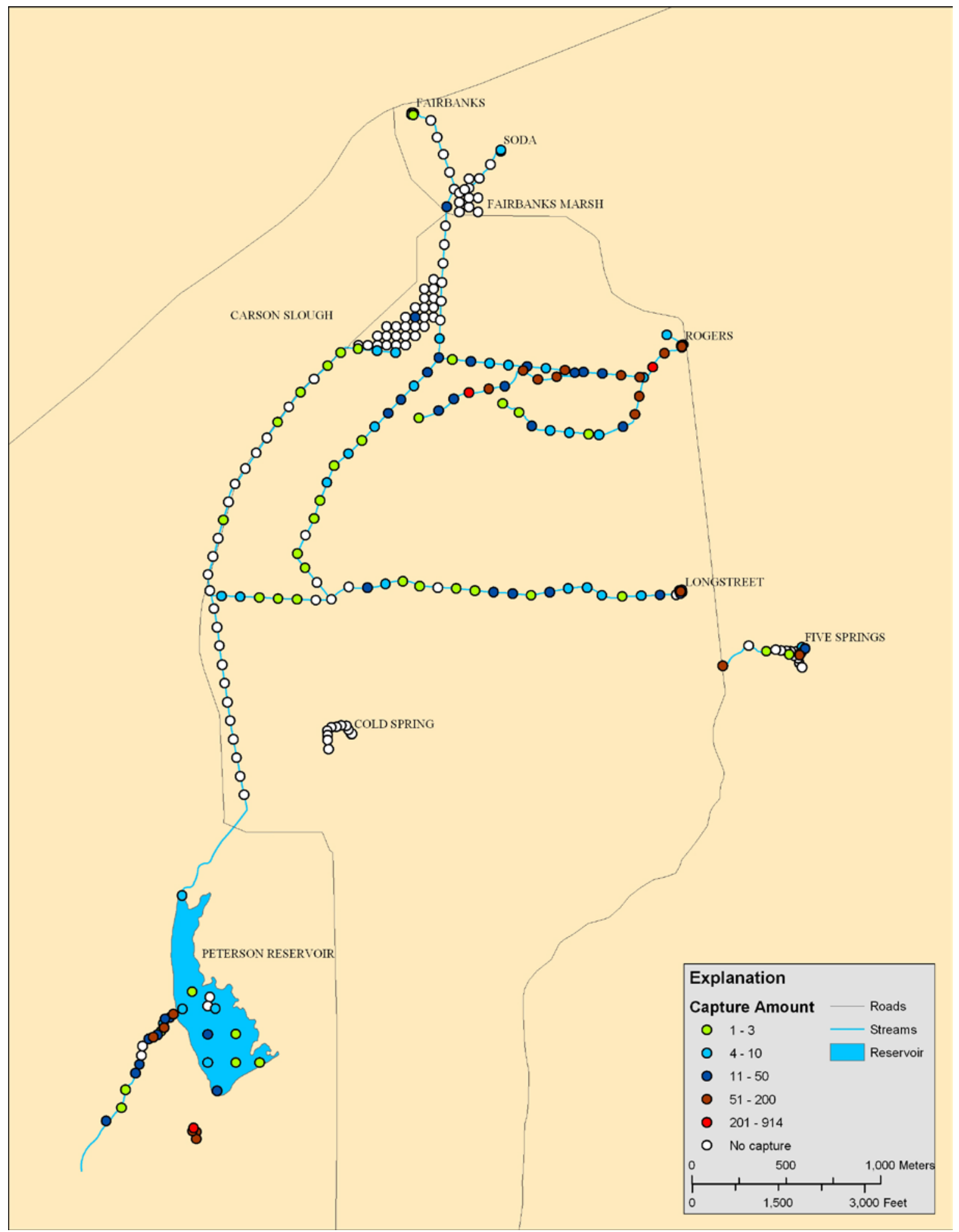

Figure A1. Composite relative abundance and seasonal distribution of mosquitofish in the northern springs, Ash Meadows National Wildlife Refuge, Nevada, fall 2007-summer 2008. 


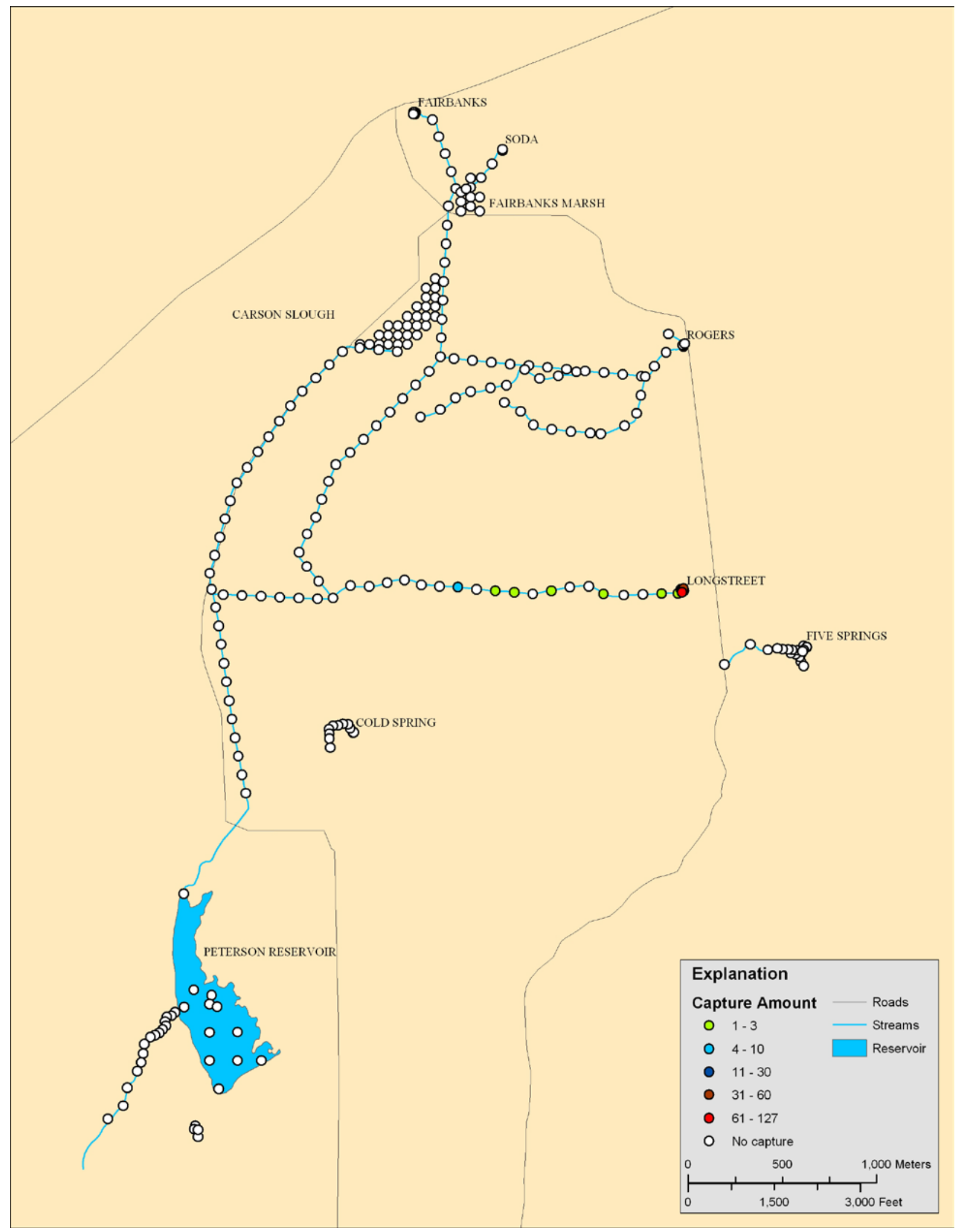

Figure A2. Composite relative abundance and seasonal distribution of sailfin mollies in the northern springs, Ash Meadows National Wildlife Refuge, Nevada, fall 2007-summer 2008. 


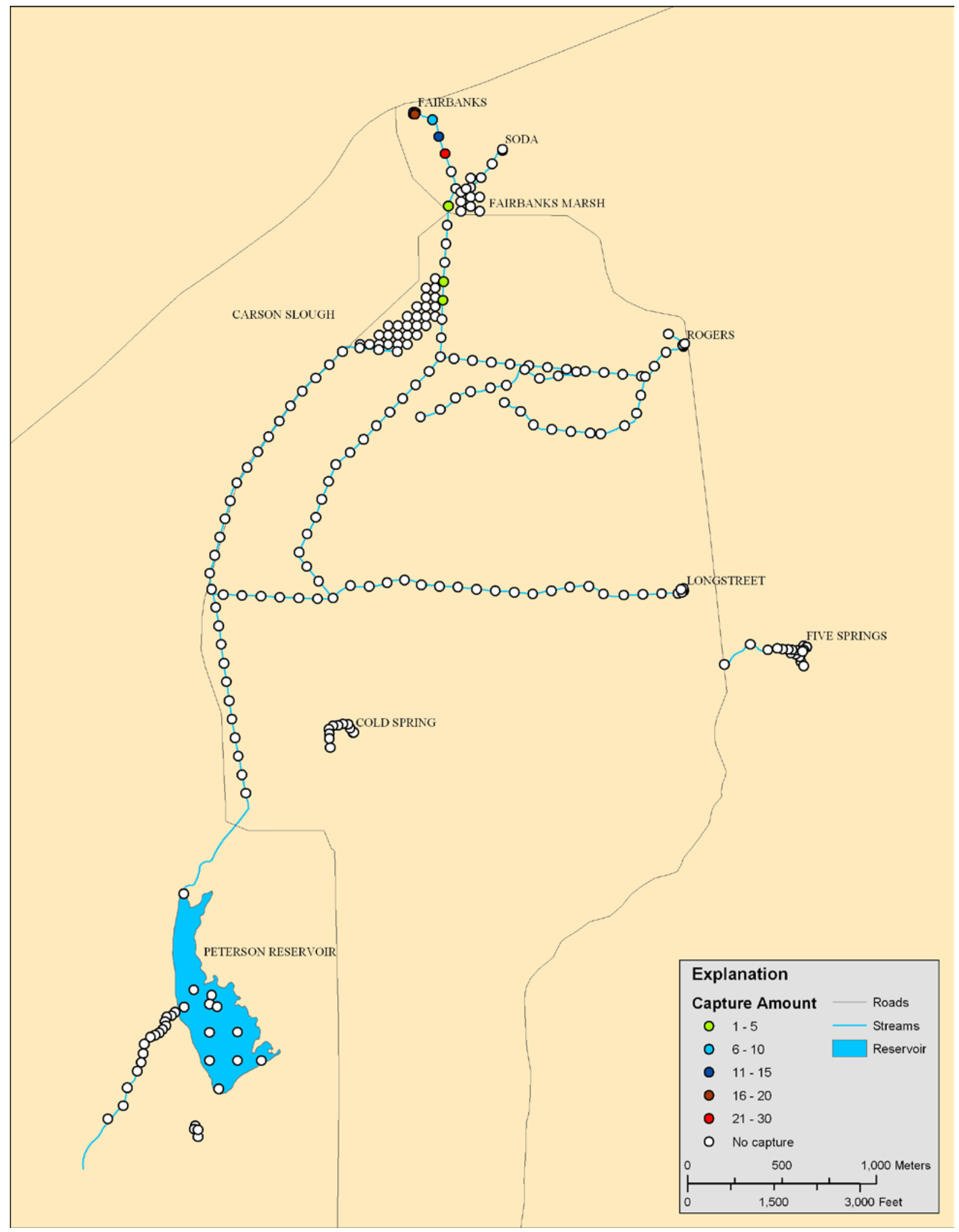

Figure A3. Composite relative abundance and seasonal distribution of convict cichlids in the northern springs, Ash Meadows National Wildlife Refuge, Nevada, fall 2007-summer 2008. 


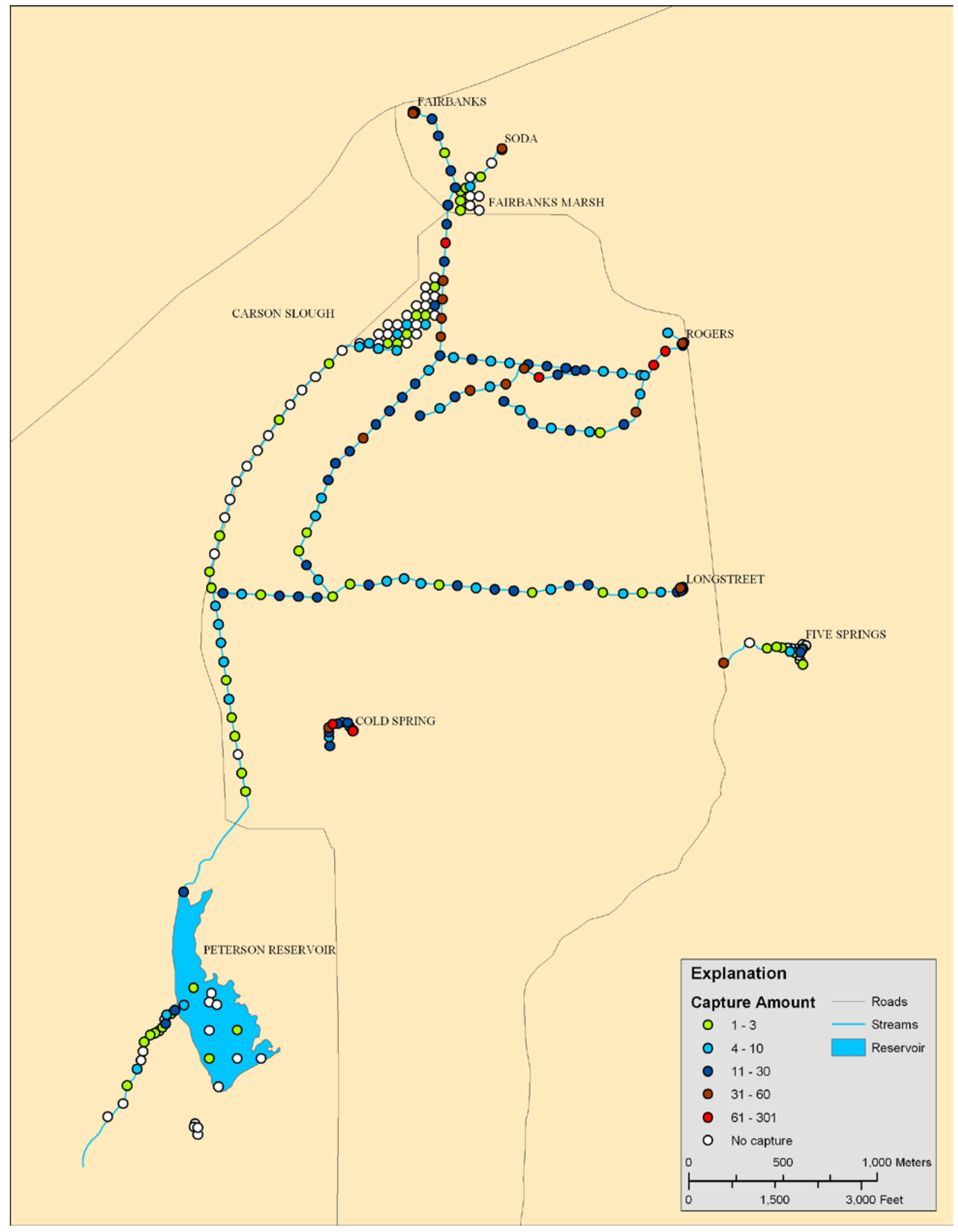

Figure A4. Composite relative abundance and seasonal distribution of crayfish in the northern springs, Ash Meadows National Wildlife Refuge, Nevada, fall 2007-summer 20 
Table A1. Species, number, efficiency, and size captured seasonally at the northern springs systems of the Ash Meadows National Wildlife Refuge, Nevada, fall 2007-summer 2008.

[Spring locations are shown in figures 1 and 2. FL, fork length. Species: CYMI, Ash Meadows Amargosa pupfish; CYPE, Warm Springs Amargosa pupfish; RHON, Ash Meadows speckled dace; GAAF, Mosquitofish; POLA, Sailfin Molly; LECY, Green Sunfish; MISA, Largemouth Bass; ARNI, Convict Cichlid; AMME, Black Bullhead; LICA, Bull Frog; PRCL, Red Swamp Crayfish]

Fall 2007

\begin{tabular}{|c|c|c|c|c|c|c|}
\hline System & Species & Total catch & Average/trap & $\mathbf{n}$ & $\begin{array}{c}\mathrm{FL} \\
(\min -\max )\end{array}$ & $\begin{array}{c}\text { Average } \pm \text { Standard } \\
\text { Deviation }\end{array}$ \\
\hline \multirow{5}{*}{ Fairbanks Spring-pool } & CYMI & 453 & 75.5 & 117 & $25-47$ & $35 \pm 5$ \\
\hline & GAAF & 1 & 0.2 & 1 & 37 & 37 \\
\hline & ARNI & 50 & 8.3 & 50 & $28-62$ & $43 \pm 8$ \\
\hline & LICA & 5 & 0.8 & 5 & $45-65$ & $57 \pm 8$ \\
\hline & PRCL & 4 & 0.7 & 4 & $54-65$ & $59 \pm 6$ \\
\hline \multirow{5}{*}{ Fairbanks stream } & CYMI & 457 & 6.9 & 175 & $17-54$ & $34 \pm 7$ \\
\hline & GAAF & 200 & 3.0 & 101 & $20-54$ & $35 \pm 8$ \\
\hline & ARNI & 72 & 1.1 & 40 & $25-65$ & $50 \pm 9$ \\
\hline & LICA & 4 & 0.1 & - & - & - \\
\hline & PRCL & 144 & 2.2 & 144 & $17-72$ & $53 \pm 12$ \\
\hline Fairbanks marsh & PRCL & 2 & 0.3 & 2 & $64-68$ & $66 \pm 3$ \\
\hline \multirow{3}{*}{ Carson Slough marsh } & CYMI & 4 & 0.4 & 4 & $27-35$ & $30 \pm 4$ \\
\hline & GAAF & 11 & 1.0 & 10 & $22-42$ & $31 \pm 6$ \\
\hline & PRCL & 50 & 4.5 & 48 & $21-72$ & $48 \pm 15$ \\
\hline \multirow{2}{*}{ Soda spring-pool } & GAAF & 8 & 8.0 & 8 & $21-31$ & $26 \pm 3$ \\
\hline & PRCL & 12 & 12.0 & 12 & $30-64$ & $46 \pm 12$ \\
\hline \multirow{2}{*}{ Soda stream } & GAAF & 3 & 1.0 & 3 & $20-28$ & $24 \pm 4$ \\
\hline & PRCL & 12 & 4.0 & 12 & $22-64$ & $46 \pm 12$ \\
\hline \multirow{4}{*}{ Rogers spring-pool } & CYMI & 410 & 68.3 & 100 & $18-42$ & $28 \pm 5$ \\
\hline & GAAF & 42 & 7.0 & 42 & $18-43$ & $25 \pm 5$ \\
\hline & LICA & 12 & 2.0 & 12 & $10-64$ & $43 \pm 16$ \\
\hline & PRCL & 12 & 2.0 & 12 & $34-53$ & $45 \pm 6$ \\
\hline \multirow{4}{*}{ Rogers stream } & CYMI & 44 & 1.3 & 39 & $15-41$ & $30 \pm 6$ \\
\hline & GAAF & 485 & 13.9 & 182 & $20-49$ & $31 \pm 7$ \\
\hline & LICA & 1 & - & 1 & 42 & 42 \\
\hline & PRCL & 214 & 6.1 & 160 & $20-75$ & $49 \pm 13$ \\
\hline \multirow{4}{*}{ Longstreet spring-pool } & CYMI & 546 & 91.0 & 50 & $20-50$ & $30 \pm 8$ \\
\hline & GAAF & 56 & 9.3 & 24 & $22-44$ & $29 \pm 6$ \\
\hline & POLA & 53 & 8.8 & 43 & $16-58$ & $34 \pm 10$ \\
\hline & PRCL & 17 & 2.8 & 17 & $33-75$ & $51 \pm 11$ \\
\hline \multirow{4}{*}{ Longstreet stream } & CYMI & 3 & 0.2 & 3 & $32-40$ & $36 \pm 4$ \\
\hline & GAAF & 7 & 0.4 & 7 & $26-43$ & $35 \pm 6$ \\
\hline & POLA & 2 & 0.1 & 2 & $28-33$ & $31 \pm 4$ \\
\hline & PRCL & 33 & 1.8 & 31 & $26-72$ & $51 \pm 12$ \\
\hline \multirow{3}{*}{$\begin{array}{l}\text { Five Springs spring- } \\
\text { pool }\end{array}$} & CYMI & 32 & 6.4 & 11 & $18-36$ & $30 \pm 5$ \\
\hline & GAAF & - & - & - & - & - \\
\hline & PRCL & 2 & 0.4 & 2 & $50-51$ & $51 \pm 1$ \\
\hline \multirow{3}{*}{ Five Springs stream } & CYMI & 4 & 0.3 & 4 & $23-25$ & $25 \pm 1$ \\
\hline & GAAF & 13 & 0.9 & 13 & $21-54$ & $33 \pm 12$ \\
\hline & PRCL & 7 & 0.5 & 7 & $32-63$ & $51 \pm 12$ \\
\hline \multirow{3}{*}{ Peterson Reservoir } & CYMI & 44 & 4.0 & 41 & $17-33$ & $24 \pm 4$ \\
\hline & GAAF & 2 & 0.2 & 2 & $23-36$ & $30 \pm 9$ \\
\hline & PRCL & 3 & 0.3 & 3 & $28-56$ & $41 \pm 14$ \\
\hline \multirow{3}{*}{ Peterson stream } & CYMI & 351 & 17.6 & 79 & $14-52$ & $28 \pm 6$ \\
\hline & GAAF & 75 & 3.8 & 43 & $18-47$ & $30 \pm 7$ \\
\hline & PRCL & 1 & 0.1 & 1 & 24 & 24 \\
\hline Cold spring-pool & PRCL & 17 & 17.0 & 17 & $14-70$ & $50 \pm 15$ \\
\hline \multirow{2}{*}{ Cold stream } & CYMI & 3 & 0.3 & 3 & $26-46$ & $37 \pm 10$ \\
\hline & PRCL & 39 & 3.9 & 35 & $19-74$ & $52 \pm 15$ \\
\hline Cold pool & PRCL & 25 & 25.0 & 10 & $23-68$ & $46 \pm 13$ \\
\hline
\end{tabular}


Table A1. Species, number, efficiency, and size captured seasonally at the northern springs systems of the Ash Meadows National Wildlife Refuge, Nevada, fall 2007-summer 2008.—Continued

\begin{tabular}{|c|c|c|c|c|c|c|}
\hline \multicolumn{7}{|c|}{ Winter 2008} \\
\hline System & Species & Total catch & Average/trap & $\mathbf{n}$ & $\begin{array}{c}\mathrm{FL} \\
(\min -\max )\end{array}$ & $\begin{array}{c}\text { Average } \pm \text { Standard } \\
\text { Deviation }\end{array}$ \\
\hline \multirow{5}{*}{$\begin{array}{c}\text { Fairbanks Spring- } \\
\text { pool }\end{array}$} & CYMI & 581 & 96.8 & 86 & $24-45$ & $34 \pm 5$ \\
\hline & GAAF & - & - & - & - & - \\
\hline & ARNI & - & - & - & - & - \\
\hline & LICA & - & - & - & - & - \\
\hline & PRCL & 18 & 3.0 & 18 & $22-61$ & $36 \pm 12$ \\
\hline \multirow{5}{*}{ Fairbanks stream } & CYMI & 149 & 3.1 & 40 & $25-45$ & $34 \pm 5$ \\
\hline & GAAF & - & - & - & - & - \\
\hline & ARNI & - & - & - & - & - \\
\hline & LICA & - & - & - & - & - \\
\hline & PRCL & 131 & 2.7 & 127 & $21-74$ & $52 \pm 12$ \\
\hline Fairbanks marsh & PRCL & - & - & - & - & - \\
\hline \multirow{3}{*}{$\begin{array}{l}\text { Carson Slough } \\
\text { marsh }\end{array}$} & CYMI & - & - & - & - & - \\
\hline & GAAF & - & - & - & - & - \\
\hline & PRCL & - & - & - & - & - \\
\hline \multirow{2}{*}{ Soda spring-pool } & GAAF & - & - & - & - & - \\
\hline & PRCL & 18 & 18.0 & 18 & $31-61$ & $47 \pm 10$ \\
\hline \multirow{2}{*}{ Soda stream } & GAAF & - & - & - & - & - \\
\hline & PRCL & 5 & 1.7 & 5 & $41-65$ & $56 \pm 9$ \\
\hline \multirow{4}{*}{ Rogers spring-pool } & CYMI & 466 & 77.7 & 103 & $15-40$ & $26 \pm 4$ \\
\hline & GAAF & 69 & 11.5 & 42 & 19-39 & $27 \pm 5$ \\
\hline & LICA & 29 & 4.8 & 0 & - & - \\
\hline & PRCL & 27 & 4.5 & 27 & $22-55$ & $41 \pm 7$ \\
\hline \multirow{4}{*}{ Rogers stream } & CYMI & 59 & 1.6 & 58 & $19-52$ & $31 \pm 6$ \\
\hline & GAAF & 123 & 3.3 & 66 & $21-44$ & $29 \pm 5$ \\
\hline & LICA & 1 & - & 1 & 115 & 115 \\
\hline & PRCL & 227 & 6.1 & 176 & $23-75$ & $50 \pm 11$ \\
\hline \multirow{4}{*}{$\begin{array}{l}\text { Longstreet spring- } \\
\text { pool }\end{array}$} & CYMI & 332 & 55.3 & 100 & $17-48$ & $31 \pm 7$ \\
\hline & GAAF & 36 & 6.0 & 36 & $21-45$ & $30 \pm 7$ \\
\hline & POLA & 19 & 3.2 & 19 & $24-54$ & $39 \pm 9$ \\
\hline & PRCL & 23 & 3.8 & 23 & $31-72$ & $49 \pm 12$ \\
\hline \multirow{4}{*}{ Longstreet stream } & CYMI & 22 & 1.2 & 22 & $20-45$ & $35 \pm 6$ \\
\hline & GAAF & 6 & 0.3 & 6 & $25-38$ & $32 \pm 5$ \\
\hline & POLA & 1 & 0.1 & 1 & 52 & 52 \\
\hline & PRCL & 22 & 1.2 & 22 & $29-64$ & $47 \pm 11$ \\
\hline \multirow{3}{*}{$\begin{array}{l}\text { Five Springs spring- } \\
\text { pool }\end{array}$} & CYMI & 21 & 5.3 & 21 & $20-34$ & $26 \pm 4$ \\
\hline & GAAF & 9 & 2.3 & 9 & $20-34$ & $29 \pm 4$ \\
\hline & PRCL & 1 & 0.3 & 1 & 62 & 62 \\
\hline \multirow{3}{*}{ Five Springs stream } & CYMI & 3 & 0.3 & 3 & $25-40$ & $33 \pm 8$ \\
\hline & GAAF & 57 & 5.2 & 19 & $23-60$ & $33 \pm 10$ \\
\hline & PRCL & 20 & 1.8 & 18 & $28-65$ & $48 \pm 11$ \\
\hline \multirow{3}{*}{ Peterson reservoir } & CYMI & 2 & 0.2 & 2 & $22-24$ & $23 \pm 1$ \\
\hline & GAAF & - & - & - & - & - \\
\hline & PRCL & 1 & 0.1 & 1 & 26 & 26 \\
\hline \multirow{3}{*}{ Peterson stream } & CYMI & 108 & 10.8 & 44 & $15-43$ & $26 \pm 6$ \\
\hline & GAAF & 5 & 0.5 & 5 & $25-47$ & $34 \pm 9$ \\
\hline & PRCL & 28 & 2.8 & 25 & $20-65$ & $33 \pm 10$ \\
\hline Cold spring-pool & PRCL & 45 & 45.0 & 20 & $27-71$ & $40 \pm 13$ \\
\hline \multirow{2}{*}{ Cold stream } & CYMI & - & - & - & - & - \\
\hline & PRCL & 113 & 11.3 & 74 & $20-68$ & $49 \pm 12$ \\
\hline Cold pool & PRCL & 32 & 32.0 & 10 & $31-68$ & $47 \pm 12$ \\
\hline
\end{tabular}


Table A1. Species, number, efficiency, and size captured seasonally at the northern springs systems of the Ash Meadows National Wildlife Refuge, Nevada, fall 2007-summer 2008._Continued

\begin{tabular}{|c|c|c|c|c|c|c|}
\hline \multicolumn{7}{|c|}{ Spring 2008} \\
\hline System & Species & Total catch & Average/trap & $\mathbf{n}$ & $\begin{array}{c}\mathrm{FL} \\
(\min -\max )\end{array}$ & $\begin{array}{c}\text { Average } \pm \text { Standard } \\
\text { Deviation }\end{array}$ \\
\hline \multirow{5}{*}{$\begin{array}{c}\text { Fairbanks Spring- } \\
\text { pool }\end{array}$} & CYMI & 358 & 59.7 & 126 & $18-57$ & $34 \pm 7$ \\
\hline & GAAF & - & - & - & - & - \\
\hline & ARNI & - & - & - & - & - \\
\hline & LICA & - & - & - & - & - \\
\hline & PRCL & 21 & 3.5 & 21 & $27-69$ & $50 \pm 10$ \\
\hline \multirow{5}{*}{ Fairbanks stream } & CYMI & 284 & 5.8 & 135 & $11-52$ & $30 \pm 9$ \\
\hline & GAAF & 2 & - & 2 & $27-47$ & $37 \pm 14$ \\
\hline & ARNI & - & - & - & - & - \\
\hline & LICA & 7 & 0.1 & 0 & - & - \\
\hline & PRCL & 196 & 4.0 & 179 & $21-78$ & $49 \pm 12$ \\
\hline Fairbanks marsh & PRCL & 6 & 0.8 & 6 & $32-69$ & $47 \pm 15$ \\
\hline \multirow{3}{*}{$\begin{array}{l}\text { Carson Slough } \\
\text { marsh }\end{array}$} & CYMI & - & - & - & - & - \\
\hline & GAAF & - & - & - & - & - \\
\hline & PRCL & - & - & - & - & - \\
\hline \multirow{2}{*}{ Soda spring-pool } & GAAF & - & - & - & - & - \\
\hline & PRCL & 13 & 13.0 & 10 & $41-62$ & $52 \pm 7$ \\
\hline \multirow{2}{*}{ Soda stream } & GAAF & - & - & - & - & - \\
\hline & PRCL & 8 & 2.7 & 8 & $32-65$ & $50 \pm 10$ \\
\hline \multirow{4}{*}{ Rogers spring-pool } & CYMI & 340 & 56.7 & 88 & $17-40$ & $27 \pm 5$ \\
\hline & GAAF & 39 & 6.5 & 20 & $19-34$ & $24 \pm 3$ \\
\hline & LICA & 9 & 1.5 & 0 & - & - \\
\hline & PRCL & 31 & 5.2 & 31 & $29-57$ & $43 \pm 7$ \\
\hline \multirow{4}{*}{ Rogers stream } & CYMI & 149 & 4.0 & 70 & $21-46$ & $32 \pm 6$ \\
\hline & GAAF & 250 & 6.8 & 151 & $20-47$ & $32 \pm 6$ \\
\hline & LICA & 2 & 0.1 & 2 & $91-92$ & $92 \pm 1$ \\
\hline & PRCL & 184 & 5.0 & 147 & $21-74$ & $51 \pm 11$ \\
\hline \multirow{4}{*}{$\begin{array}{c}\text { Longstreet spring- } \\
\text { pool }\end{array}$} & CYMI & 353 & 58.8 & 101 & $18-53$ & $33 \pm 7$ \\
\hline & GAAF & 1 & 0.2 & 1 & 31 & 31 \\
\hline & POLA & 45 & 7.5 & 38 & $20-52$ & $34 \pm 8$ \\
\hline & PRCL & 23 & 3.8 & 23 & $37-67$ & $51 \pm 9$ \\
\hline \multirow{4}{*}{ Longstreet stream } & CYMI & 95 & 5.0 & 66 & $20-57$ & $36 \pm 7$ \\
\hline & GAAF & 39 & 2.1 & 36 & $21-47$ & $33 \pm 7$ \\
\hline & POLA & 5 & 0.3 & 5 & $20-50$ & $33 \pm 12$ \\
\hline & PRCL & 53 & 2.8 & 53 & $27-70$ & $51 \pm 11$ \\
\hline \multirow{3}{*}{$\begin{array}{l}\text { Five Springs spring- } \\
\text { pool }\end{array}$} & CYMI & 23 & 4.6 & 23 & $22-33$ & $26 \pm 3$ \\
\hline & GAAF & 5 & 1.0 & 5 & $23-31$ & $26 \pm 3$ \\
\hline & PRCL & 5 & 1.0 & 5 & $47-51$ & $49 \pm 2$ \\
\hline \multirow{3}{*}{ Five Springs stream } & CYMI & 13 & 1.0 & 13 & $22-33$ & $27 \pm 4$ \\
\hline & GAAF & 75 & 5.8 & 21 & $22-52$ & $29 \pm 9$ \\
\hline & PRCL & 9 & 0.7 & 9 & $20-68$ & $44 \pm 18$ \\
\hline \multirow{3}{*}{ Peterson reservoir } & CYMI & 12 & 1.1 & 12 & $20-33$ & $27 \pm 4$ \\
\hline & GAAF & - & - & - & - & - \\
\hline & PRCL & 4 & 0.4 & 4 & $46-75$ & $57 \pm 13$ \\
\hline \multirow{3}{*}{ Peterson stream } & CYMI & 861 & 43.1 & 172 & $19-43$ & $31 \pm 5$ \\
\hline & GAAF & 11 & 0.6 & 10 & $27-62$ & $38 \pm 10$ \\
\hline & PRCL & 8 & 0.4 & 8 & $42-83$ & $52 \pm 13$ \\
\hline Cold spring-pool & PRCL & 32 & 32.0 & 9 & $33-67$ & $50 \pm 13$ \\
\hline \multirow{2}{*}{ Cold stream } & CYMI & 4 & 0.5 & 4 & $28-37$ & $33 \pm 4$ \\
\hline & PRCL & 54 & 6.8 & 44 & $27-67$ & $49 \pm 9$ \\
\hline Cold pool & PRCL & 18 & 18.0 & 10 & $23-67$ & $45 \pm 11$ \\
\hline
\end{tabular}


Table A1. Species, number, efficiency, and size captured seasonally at the northern springs systems of the Ash Meadows National Wildlife Refuge, Nevada, fall 2007-summer 2008.-Continued

\begin{tabular}{|c|c|c|c|c|c|c|}
\hline \multicolumn{7}{|c|}{ Summer 2008} \\
\hline System & Species & Total catch & Average/trap & $\mathbf{n}$ & $\begin{array}{c}\mathrm{FL} \\
(\min -\max )\end{array}$ & $\begin{array}{c}\text { Average } \pm \text { Standard } \\
\text { Deviation }\end{array}$ \\
\hline \multirow{5}{*}{$\begin{array}{c}\text { Fairbanks Spring- } \\
\text { pool }\end{array}$} & CYMI & 821 & 136.8 & 133 & $17-55$ & $32 \pm 6$ \\
\hline & GAAF & - & - & - & - & - \\
\hline & ARNI & - & - & - & - & - \\
\hline & LICA & - & - & - & - & - \\
\hline & PRCL & 35 & 5.8 & 15 & $42-73$ & $63 \pm 8$ \\
\hline \multirow{5}{*}{ Fairbanks stream } & CYMI & 2,235 & 45.6 & 341 & $18-50$ & $31 \pm 6$ \\
\hline & GAAF & 45 & 0.9 & 38 & $21-45$ & $31 \pm 7$ \\
\hline & ARNI & - & - & - & - & - \\
\hline & LICA & - & - & - & - & - \\
\hline & PRCL & 295 & 6.0 & 165 & $24-73$ & $52 \pm 11$ \\
\hline Fairbanks marsh & PRCL & - & - & - & - & - \\
\hline \multirow{3}{*}{$\begin{array}{l}\text { Carson Slough } \\
\text { marsh }\end{array}$} & CYMI & - & - & - & - & - \\
\hline & GAAF & - & - & - & - & - \\
\hline & PRCL & - & - & - & - & - \\
\hline \multirow{2}{*}{ Soda spring-pool } & GAAF & - & - & - & - & - \\
\hline & PRCL & 16 & 16.0 & 16 & $30-64$ & $49 \pm 10$ \\
\hline \multirow{2}{*}{ Soda stream } & GAAF & - & - & - & - & - \\
\hline & PRCL & 3 & 1.5 & 3 & $27-60$ & $48 \pm 19$ \\
\hline \multirow{4}{*}{ Rogers spring-pool } & CYMI & 341 & 56.8 & 125 & $19-41$ & $28 \pm 4$ \\
\hline & GAAF & 248 & 41.3 & 95 & $15-36$ & $26 \pm 6$ \\
\hline & LICA & 2 & 0.3 & 0 & - & - \\
\hline & PRCL & 32 & 5.3 & 31 & $30-65$ & $45 \pm 9$ \\
\hline \multirow{4}{*}{ Rogers stream } & CYMI & 60 & 1.8 & 60 & $15-46$ & $31 \pm 6$ \\
\hline & GAAF & 919 & 27.8 & 230 & $19-47$ & $33 \pm 6$ \\
\hline & LICA & - & - & - & - & - \\
\hline & PRCL & 144 & 4.4 & 114 & $20-72$ & $52 \pm 11$ \\
\hline \multirow{4}{*}{$\begin{array}{l}\text { Longstreet spring- } \\
\text { pool }\end{array}$} & CYMI & 458 & 76.3 & 132 & $16-52$ & $29 \pm 7$ \\
\hline & GAAF & 80 & 13.3 & 72 & $18-50$ & $28 \pm 7$ \\
\hline & POLA & 133 & 22.2 & 112 & $16-52$ & $33 \pm 8$ \\
\hline & PRCL & 69 & 11.5 & 62 & $15-69$ & $44 \pm 11$ \\
\hline \multirow{4}{*}{ Longstreet stream } & CYMI & 98 & 5.4 & 87 & $16-45$ & $27 \pm 8$ \\
\hline & GAAF & 73 & 4.1 & 62 & $21-45$ & $33 \pm 7$ \\
\hline & POLA & 4 & 0.2 & 4 & $25-50$ & $37 \pm 11$ \\
\hline & PRCL & 68 & 3.8 & 62 & $22-72$ & $52 \pm 11$ \\
\hline \multirow{3}{*}{$\begin{array}{l}\text { Five Springs spring- } \\
\text { pool }\end{array}$} & CYMI & 21 & 4.2 & 21 & $15-36$ & $26 \pm 6$ \\
\hline & GAAF & 3 & 0.6 & 3 & $23-27$ & $25 \pm 2$ \\
\hline & PRCL & - & - & - & - & - \\
\hline \multirow{3}{*}{ Five Springs stream } & CYMI & 46 & 3.3 & 12 & $23-38$ & $31 \pm 5$ \\
\hline & GAAF & 68 & 4.9 & 22 & $18-50$ & $29 \pm 7$ \\
\hline & PRCL & 41 & 2.9 & 28 & $24-70$ & $52 \pm 13$ \\
\hline \multirow{3}{*}{ Peterson reservoir } & CYMI & 157 & 14.3 & 60 & $13-41$ & $23 \pm 7$ \\
\hline & GAAF & 95 & 8.6 & 41 & $18-40$ & $26 \pm 6$ \\
\hline & PRCL & 3 & 0.3 & 3 & $48-73$ & $63 \pm 13$ \\
\hline \multirow{3}{*}{ Peterson stream } & CYMI & 514 & 27.1 & 137 & $17-43$ & $29 \pm 5$ \\
\hline & GAAF & 1164 & 61.3 & 150 & $15-62$ & $34 \pm 9$ \\
\hline & PRCL & 20 & 1.1 & 19 & $43-73$ & $65 \pm 8$ \\
\hline Cold spring-pool & PRCL & 33 & 33.0 & 10 & $38-70$ & $56 \pm 11$ \\
\hline \multirow{2}{*}{ Cold stream } & CYMI & - & - & - & - & - \\
\hline & PRCL & 68 & 8.5 & 59 & $12-70$ & $52 \pm 11$ \\
\hline Cold pool & PRCL & 16 & 16.0 & 10 & $36-61$ & $49 \pm 8$ \\
\hline
\end{tabular}




\section{Appendix B. Seasonal Distributions of Fishes and Crayfish at Warm Springs Complex, Ash Meadows National Wildlife Refuge, Nevada}

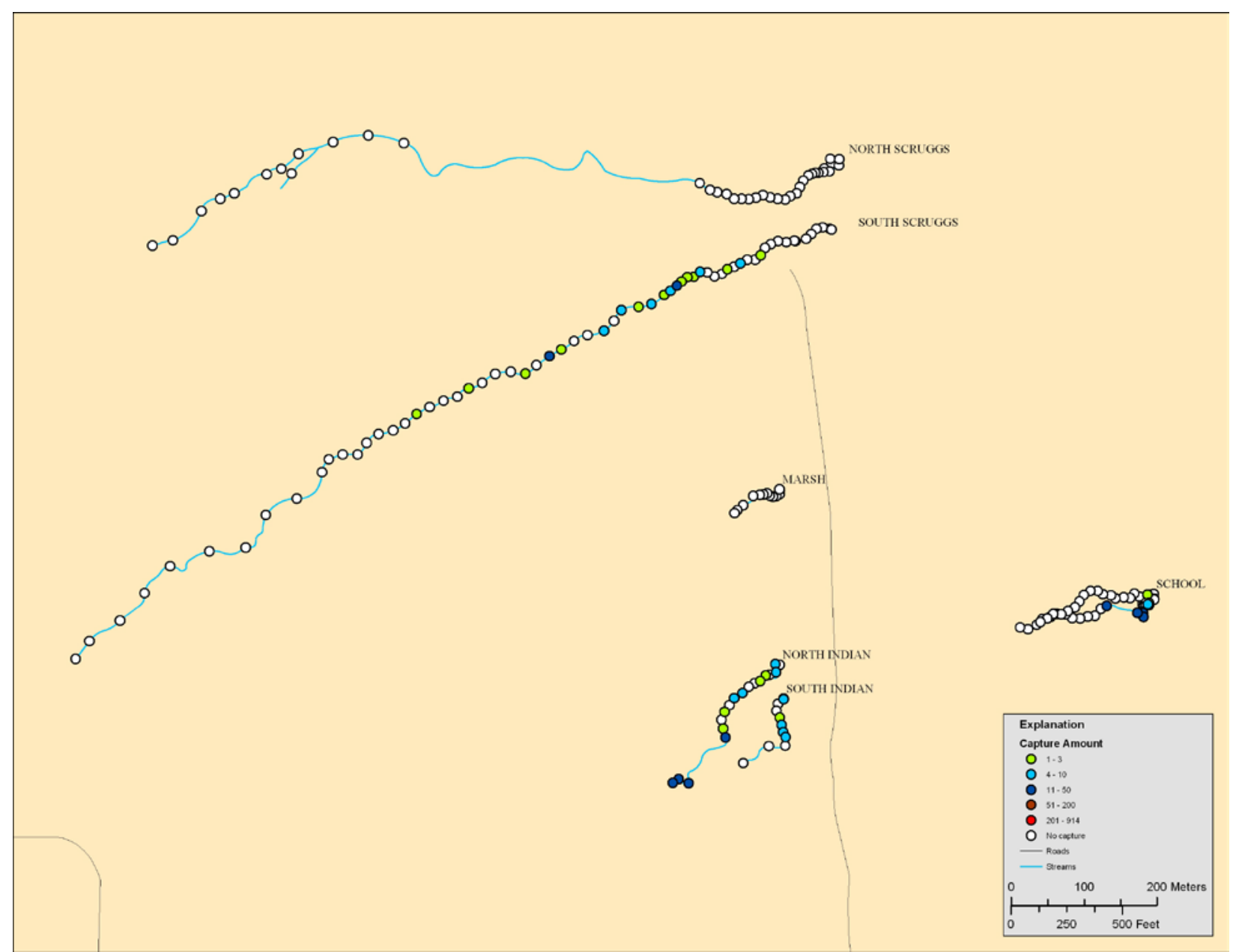

Figure B1. Composite relative abundance and seasonal distribution of mosquitofish in the Warm Springs Complex, Ash Meadows National Wildlife Refuge, Nevada, fall 2007-summer 2008. 


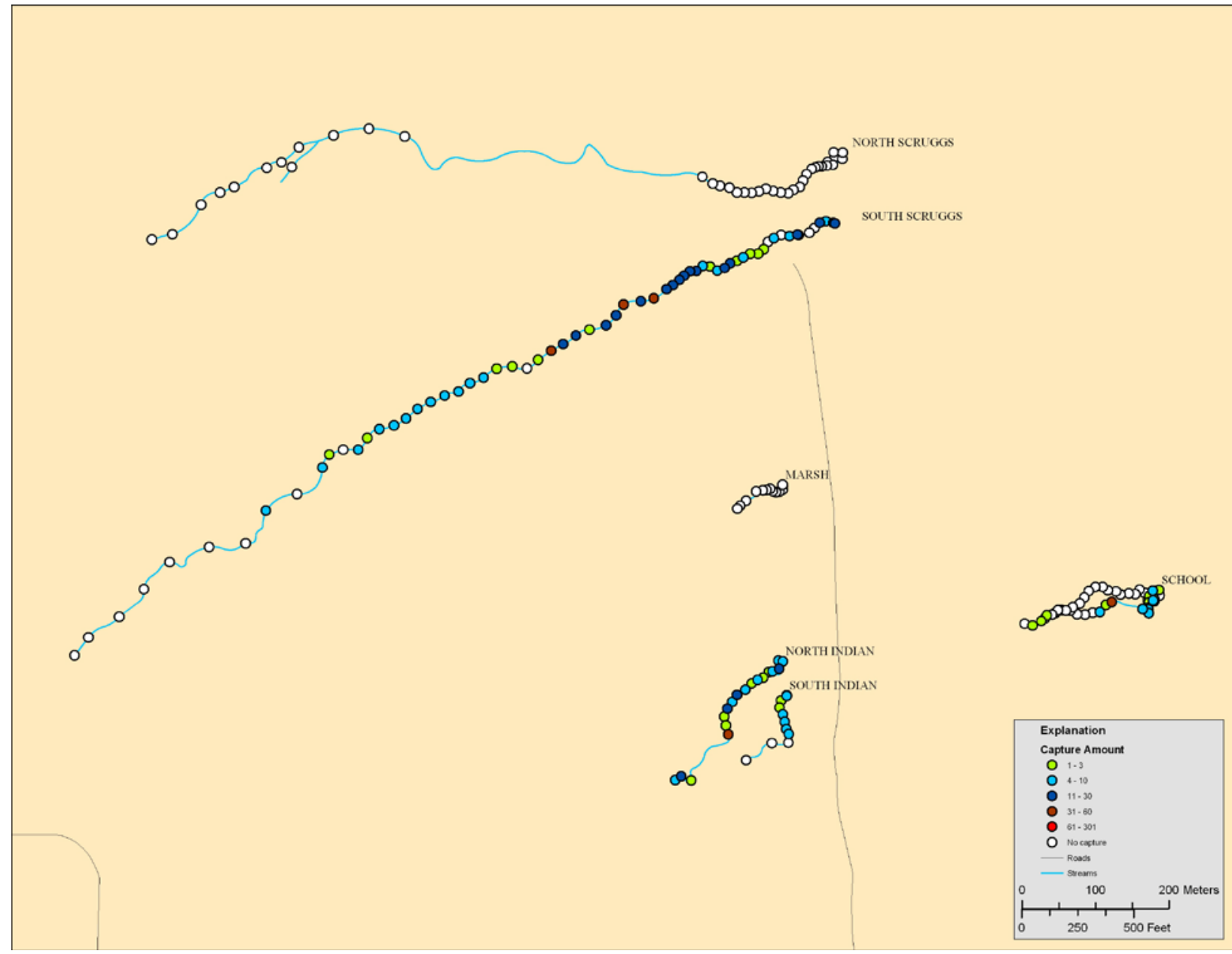

Figure B2. Composite relative abundance and seasonal distribution of crayfish in the Warm Springs Complex, Ash Meadows National Wildlife Refuge, Nevada, fall 2007-summer 2008. 
Table B1. Species, number, efficiency, and size captured seasonally at the Warm Springs Complex of the Ash Meadows National Wildlife Refuge, Nevada, fall 2007-summer 2008.

[Spring locations are shown in figures 1 and 2. FL, fork length. Species: CYMI, Ash Meadows Amargosa pupfish; CYPE, Warm Springs Amargosa pupfish; RHON, Ash Meadows speckled dace; GAAF, Mosquitofish; POLA, Sailfin Molly; LECY, Green Sunfish; MISA, Largemouth Bass; ARNI, Convict Cichlid; AMME, Black Bullhead; LICA, Bull Frog; PRCL, Red Swamp Crayfish]

Fall 2007

\begin{tabular}{|c|c|c|c|c|c|c|}
\hline System & Species & Total catch & Average/trap & $\mathbf{n}$ & $\begin{array}{c}\text { FL } \\
(\min -\max )\end{array}$ & $\begin{array}{c}\text { Average } \pm \text { Standard } \\
\text { Deviation }\end{array}$ \\
\hline $\begin{array}{c}\text { North Scruggs } \\
\text { spring-pool }\end{array}$ & CYPE & - & - & - & - & - \\
\hline $\begin{array}{c}\text { North Scruggs } \\
\text { stream }\end{array}$ & CYPE & 86 & 3.4 & 85 & $13-41$ & $28 \pm 6$ \\
\hline \multirow{2}{*}{$\begin{array}{l}\text { South Scruggs } \\
\text { spring-pool }\end{array}$} & CYPE & 17 & 17.0 & 17 & $17-27$ & $21 \pm 3$ \\
\hline & PRCL & 6 & 6.0 & 6 & $34-66$ & $47 \pm 11$ \\
\hline \multirow{3}{*}{$\begin{array}{c}\text { South Scruggs } \\
\text { stream }\end{array}$} & CYPE & 40 & 0.7 & 40 & $12-48$ & $28 \pm 10$ \\
\hline & GAAF & 16 & 0.3 & 16 & $12-42$ & $30 \pm 8$ \\
\hline & PRCL & 157 & 2.9 & 157 & $10-81$ & $46 \pm 12$ \\
\hline Marsh spring-pool & CYPE & - & - & - & - & - \\
\hline Marsh stream & CYPE & 168 & 14.0 & 72 & $22-44$ & $31 \pm 5$ \\
\hline \multirow{2}{*}{$\begin{array}{l}\text { North Indian } \\
\text { spring-pool }\end{array}$} & CYPE & - & - & - & - & - \\
\hline & PRCL & 3 & 3.0 & 3 & $16-60$ & $36 \pm 22$ \\
\hline \multirow{4}{*}{$\begin{array}{l}\text { North Indian } \\
\text { stream }\end{array}$} & CYPE & 79 & 4.4 & 37 & $17-45$ & $29 \pm 7$ \\
\hline & GAAF & 62 & 3.4 & 47 & $19-48$ & $30 \pm 6$ \\
\hline & LICA & - & - & - & - & - \\
\hline & PRCL & 55 & 3.1 & 48 & $17-68$ & $44 \pm 14$ \\
\hline \multirow{2}{*}{$\begin{array}{c}\text { South Indian } \\
\text { stream }\end{array}$} & GAAF & 22 & 3.1 & 22 & $21-42$ & $28 \pm 5$ \\
\hline & PRCL & 15 & 2.1 & 15 & $27-66$ & $50 \pm 11$ \\
\hline \multirow{3}{*}{$\begin{array}{l}\text { School spring- } \\
\text { pool }\end{array}$} & CYPE & 19 & 9.5 & 19 & $17-30$ & $23 \pm 4$ \\
\hline & GAAF & 3 & 1.5 & 3 & $18-27$ & $23 \pm 5$ \\
\hline & PRCL & 4 & 2.0 & 4 & $45-59$ & $55 \pm 6$ \\
\hline \multirow{3}{*}{ School ponds } & CYPE & 294 & 32.7 & 92 & $17-41$ & $27 \pm 6$ \\
\hline & GAAF & 50 & 5.6 & 38 & $21-55$ & $32 \pm 8$ \\
\hline & PRCL & 21 & 2.3 & 21 & $32-70$ & $51 \pm 11$ \\
\hline \multirow{3}{*}{ School stream } & CYPE & 62 & 3.9 & 62 & $15-45$ & $25 \pm 6$ \\
\hline & GAAF & 40 & 2.5 & 18 & $23-38$ & $28 \pm 5$ \\
\hline & PRCL & 41 & 2.6 & 31 & $13-72$ & $47 \pm 17$ \\
\hline
\end{tabular}


Table B1. Species, number, efficiency, and size captured seasonally at the Warm Springs Complex of the Ash Meadows National Wildlife Refuge, Nevada. - Continued

\begin{tabular}{|c|c|c|c|c|c|c|}
\hline \multicolumn{7}{|c|}{ Winter 2008} \\
\hline System & Species & Total catch & Average/trap & $\mathbf{n}$ & $\begin{array}{c}\mathrm{FL} \\
(\min -\max )\end{array}$ & $\begin{array}{c}\text { Average } \pm \text { Standard } \\
\text { Deviation }\end{array}$ \\
\hline North Scruggs spring-pool & CYPE & - & - & - & - & - \\
\hline North Scruggs stream & CYPE & 84 & 2.8 & 71 & $15-45$ & $32 \pm 7$ \\
\hline \multirow{2}{*}{ South Scruggs spring-pool } & CYPE & - & - & - & - & - \\
\hline & PRCL & 1 & 1.0 & 1 & 43 & 43 \\
\hline \multirow{3}{*}{ South Scruggs stream } & CYPE & 57 & 1.0 & 57 & $14-46$ & $28 \pm 9$ \\
\hline & GAAF & 14 & 0.3 & 14 & $18-40$ & $29 \pm 6$ \\
\hline & PRCL & 131 & 2.3 & 112 & $18-68$ & $44 \pm 11$ \\
\hline Marsh spring-pool & CYPE & - & - & - & - & - \\
\hline Marsh stream & CYPE & 171 & 14.3 & 72 & $20-46$ & $32 \pm 6$ \\
\hline \multirow{2}{*}{ North Indian spring-pool } & CYPE & 1 & 1.0 & 1 & 29 & 29 \\
\hline & PRCL & 4 & 4.0 & 4 & $32-68$ & $44 \pm 16$ \\
\hline \multirow{4}{*}{ North Indian stream } & CYPE & 34 & 2.0 & 23 & $19-43$ & $28 \pm 7$ \\
\hline & GAAF & 17 & 1.0 & 17 & $21-36$ & $28 \pm 4$ \\
\hline & LICA & 2 & 0.1 & 2 & $50-51$ & $51 \pm 1$ \\
\hline & PRCL & 34 & 2.0 & 31 & $21-61$ & $42 \pm 10$ \\
\hline \multirow{2}{*}{ South Indian stream } & GAAF & 2 & 0.3 & 2 & $35-37$ & $36 \pm 1$ \\
\hline & PRCL & 9 & 1.1 & 9 & $25-59$ & $44 \pm 11$ \\
\hline \multirow{3}{*}{ School spring-pool } & CYPE & 4 & 2.0 & 4 & $18-22$ & $20 \pm 2$ \\
\hline & GAAF & - & - & - & - & - \\
\hline & PRCL & - & - & - & - & - \\
\hline \multirow{3}{*}{ School ponds } & CYPE & 267 & 44.5 & 120 & $16-42$ & $26 \pm 6$ \\
\hline & GAAF & 45 & 7.5 & 40 & $20-55$ & $31 \pm 8$ \\
\hline & PRCL & 3 & 0.5 & 2 & 65 & 65 \\
\hline \multirow{3}{*}{ School stream } & CYPE & 116 & 11.6 & 80 & $18-44$ & $25 \pm 6$ \\
\hline & GAAF & 24 & 2.4 & 22 & $21-41$ & $27 \pm 8$ \\
\hline & PRCL & 43 & 4.3 & 14 & $31-72$ & $42 \pm 10$ \\
\hline
\end{tabular}


Table B1. Species, number, efficiency, and size captured seasonally at the Warm Springs Complex of the Ash Meadows National Wildlife Refuge, Nevada.-Continued

\begin{tabular}{|c|c|c|c|c|c|c|}
\hline \multicolumn{7}{|c|}{ Spring 2008} \\
\hline System & Species & Total catch & Average/trap & $\mathbf{n}$ & $\begin{array}{c}\mathrm{FL} \\
(\min -\max )\end{array}$ & $\begin{array}{c}\text { Average } \pm \text { Standard } \\
\text { Deviation }\end{array}$ \\
\hline North Scruggs spring-pool & CYPE & 7 & 3.5 & 7 & $21-38$ & $26 \pm 6$ \\
\hline North Scruggs stream & CYPE & 81 & 3.0 & 81 & $17-48$ & $29 \pm 8$ \\
\hline \multirow{2}{*}{ South Scruggs spring-pool } & CYPE & 4 & 4.0 & 4 & $22-24$ & $23 \pm 1$ \\
\hline & PRCL & 4 & 4.0 & 4 & $50-58$ & $56 \pm 4$ \\
\hline \multirow{3}{*}{ South Scruggs stream } & CYPE & 52 & 0.9 & 51 & $16-49$ & $26 \pm 8$ \\
\hline & GAAF & 37 & 0.7 & 29 & $20-52$ & $31 \pm 9$ \\
\hline & PRCL & 119 & 2.2 & 119 & $22-65$ & $45 \pm 12$ \\
\hline Marsh spring-pool & CYPE & 1 & 1.0 & 1 & 38 & 38 \\
\hline Marsh stream & CYPE & 141 & 11.8 & 64 & $18-46$ & $31 \pm 7$ \\
\hline \multirow{2}{*}{ North Indian spring-pool } & CYPE & 1 & 1.0 & 1 & 30 & 30 \\
\hline & PRCL & - & - & - & - & - \\
\hline \multirow{4}{*}{ North Indian stream } & CYPE & 35 & 2.1 & 14 & $18-35$ & $29 \pm 5$ \\
\hline & GAAF & 15 & 0.9 & 15 & $21-48$ & $32 \pm 7$ \\
\hline & LICA & - & - & - & - & - \\
\hline & PRCL & 28 & 1.6 & 27 & $27-61$ & $44 \pm 10$ \\
\hline \multirow{2}{*}{ South Indian stream } & GAAF & 3 & 0.4 & 3 & $22-35$ & $27 \pm 7$ \\
\hline & PRCL & 4 & 0.6 & 4 & $42-58$ & $49 \pm 7$ \\
\hline \multirow{3}{*}{ School spring-pool } & CYPE & - & - & - & - & - \\
\hline & GAAF & - & - & - & - & - \\
\hline & PRCL & - & - & - & - & - \\
\hline \multirow{3}{*}{ School ponds } & CYPE & - & - & - & - & - \\
\hline & GAAF & - & - & - & - & - \\
\hline & PRCL & - & - & - & - & - \\
\hline \multirow{3}{*}{ School stream } & CYPE & - & - & - & - & - \\
\hline & GAAF & - & - & - & - & - \\
\hline & PRCL & - & - & - & - & - \\
\hline
\end{tabular}


Table B1. Species, number, efficiency, and size captured seasonally at the Warm Springs Complex of the Ash Meadows National Wildlife Refuge, Nevada.-Continued

\begin{tabular}{|c|c|c|c|c|c|c|}
\hline \multicolumn{7}{|c|}{ Summer 2008} \\
\hline System & Species & Total catch & Average/trap & $\mathbf{n}$ & $\begin{array}{c}\mathrm{FL} \\
(\min -\max )\end{array}$ & $\begin{array}{c}\text { Average } \pm \text { Standard } \\
\text { Deviation }\end{array}$ \\
\hline North Scruggs spring-pool & CYPE & 16 & 8.0 & 16 & $18-35$ & $26 \pm 5$ \\
\hline North Scruggs stream & CYPE & 115 & 5.5 & 115 & $17-45$ & $26 \pm 7$ \\
\hline \multirow{2}{*}{ South Scruggs spring-pool } & CYPE & 2 & 2.0 & 2 & $18-21$ & $20 \pm 2$ \\
\hline & PRCL & 9 & 9.0 & 9 & $37-68$ & $55 \pm 9$ \\
\hline \multirow{3}{*}{ South Scruggs stream } & CYPE & 51 & 0.9 & 33 & $17-47$ & $31 \pm 9$ \\
\hline & GAAF & 21 & 0.4 & 20 & 19-38 & $29 \pm 6$ \\
\hline & PRCL & 103 & 1.9 & 102 & $15-64$ & $45 \pm 11$ \\
\hline Marsh spring-pool & CYPE & - & - & - & - & - \\
\hline Marsh stream & CYPE & 152 & 12.7 & 82 & $17-50$ & $32 \pm 6$ \\
\hline \multirow{2}{*}{ North Indian spring-pool } & CYPE & 1 & 1.0 & 1 & 33 & 33 \\
\hline & PRCL & 3 & 3.0 & 3 & $47-52$ & $50 \pm 3$ \\
\hline \multirow{4}{*}{ North Indian stream } & CYPE & 32 & 2.1 & 16 & $26-39$ & $33 \pm 4$ \\
\hline & GAAF & 4 & 0.3 & 4 & $28-37$ & $32 \pm 4$ \\
\hline & LICA & - & - & - & - & - \\
\hline & PRCL & 23 & 1.5 & 22 & $24-53$ & $38 \pm 8$ \\
\hline \multirow{2}{*}{ South Indian stream } & GAAF & 3 & 0.4 & 3 & $32-37$ & $34 \pm 3$ \\
\hline & PRCL & 5 & 0.6 & 5 & $47-64$ & $57 \pm 7$ \\
\hline \multirow{3}{*}{ School spring-pool } & CYPE & 6 & 3.0 & 6 & $23-29$ & $26 \pm 2$ \\
\hline & GAAF & - & - & - & - & - \\
\hline & PRCL & - & - & - & - & - \\
\hline \multirow{3}{*}{ School ponds } & CYPE & - & - & - & - & - \\
\hline & GAAF & - & - & - & - & - \\
\hline & PRCL & - & - & - & - & - \\
\hline \multirow{3}{*}{ School stream } & CYPE & 177 & 16.1 & 70 & $16-46$ & $29 \pm 6$ \\
\hline & GAAF & - & - & - & - & - \\
\hline & PRCL & - & - & - & - & - \\
\hline
\end{tabular}




\section{Appendix C. Seasonal Distributions of Fishes and Crayfish in Southern Springs, Ash Meadows National Wildlife Refuge, Nevada}

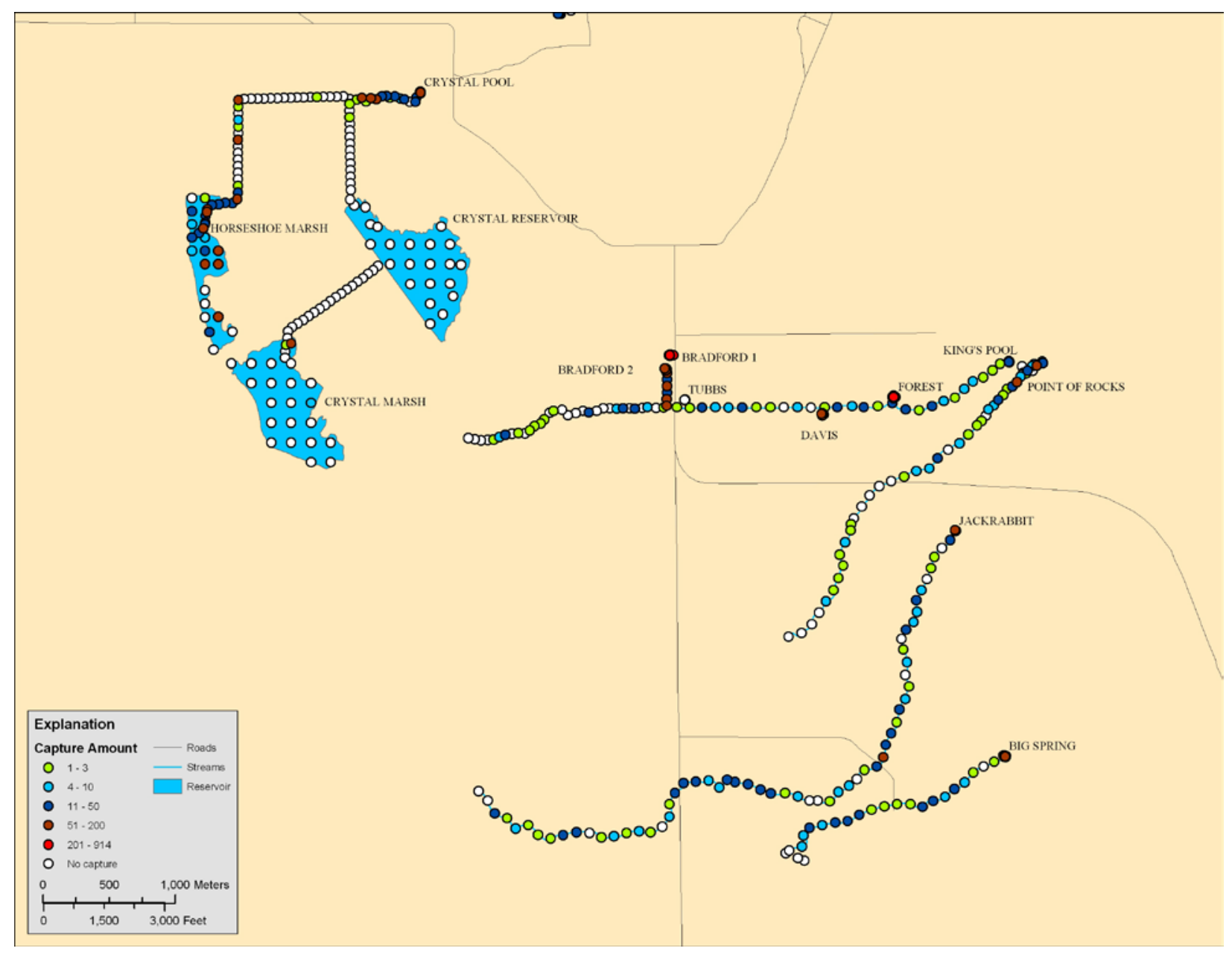

Figure C1. Composite relative abundance and seasonal distribution of mosquitofish in the southern springs, Ash Meadows National Wildlife Refuge, Nevada, fall 2007-summer 2008. 


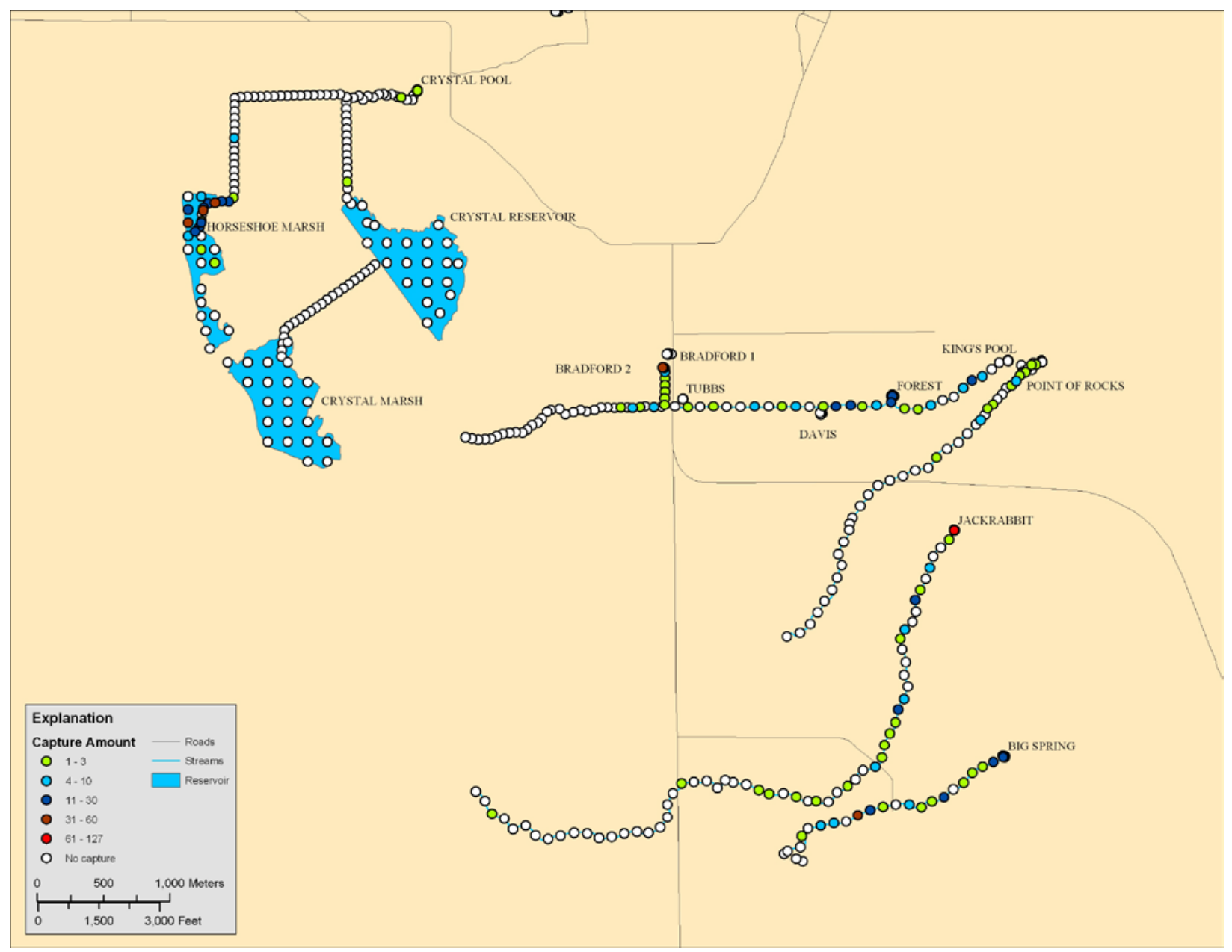

Figure C2. Composite relative abundance and seasonal distribution of salfin mollies in the southern springs, Ash Meadows National Wildlife Refuge, Nevada, fall 2007-summer 2008. 


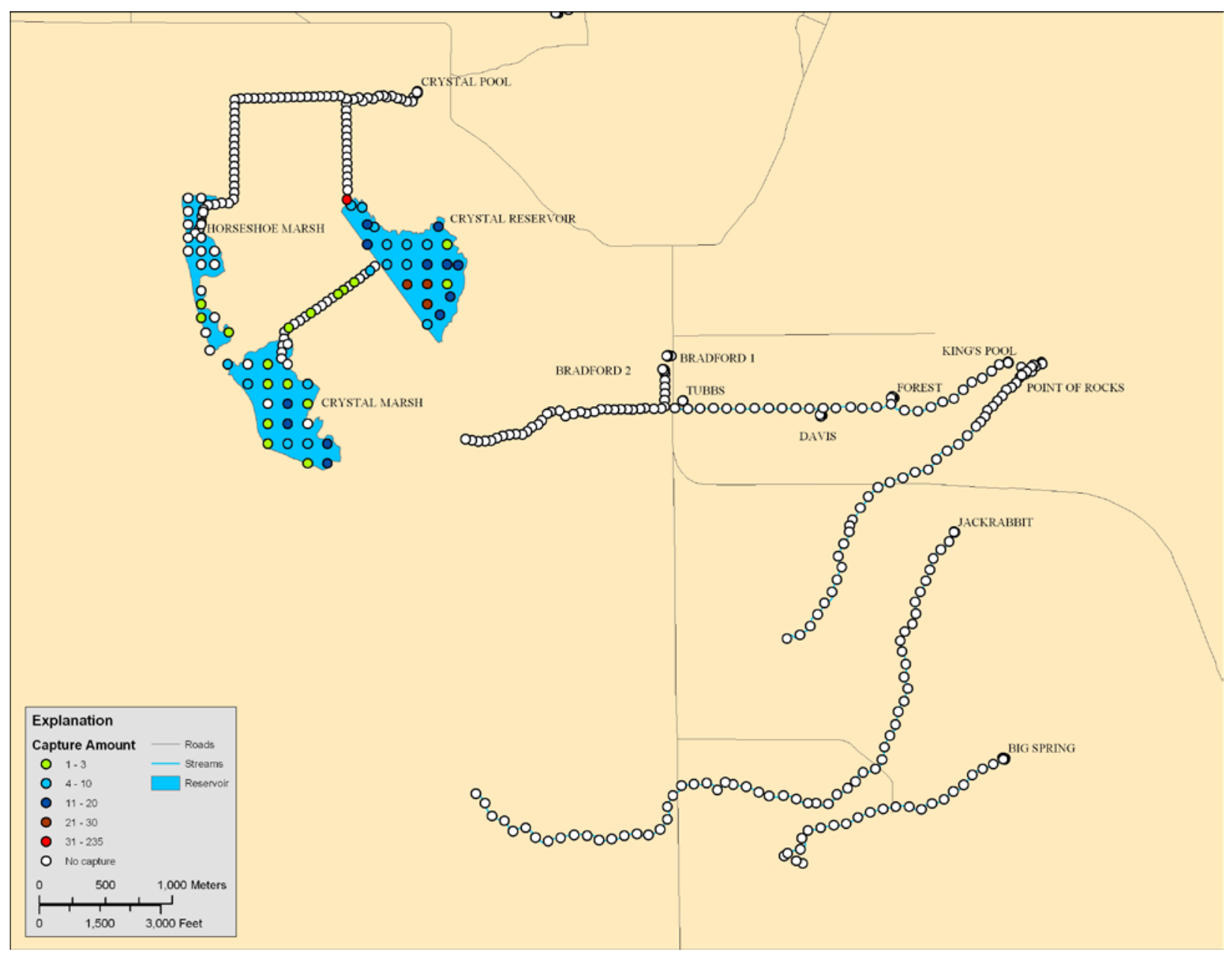

Figure C3. Composite relative abundance and seasonal distribution of green sunfish in the southern springs, Ash Meadows National Wildlife Refuge, Nevada, fall 2007-summer 2008. 


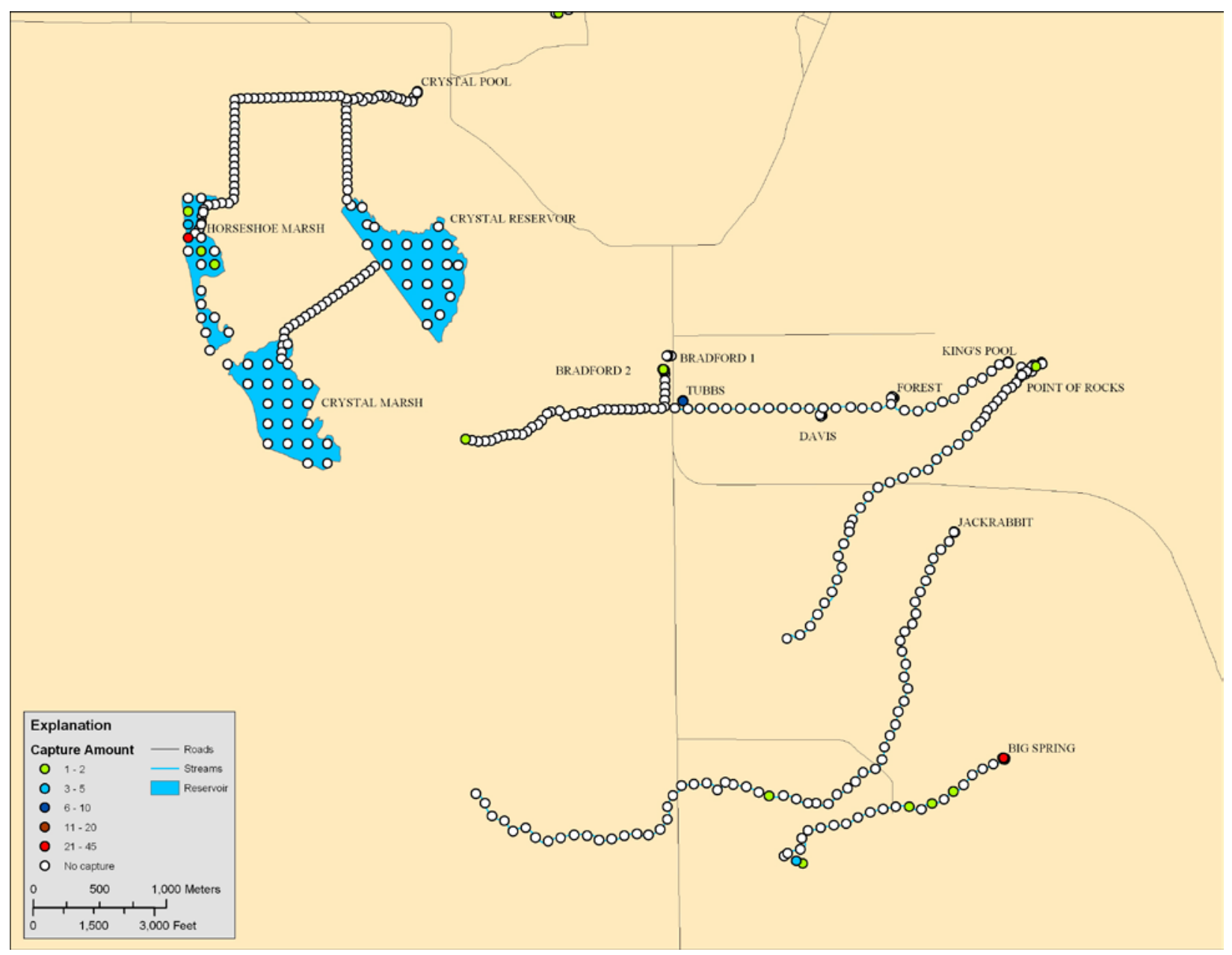

Figure C4. Composite relative abundance and seasonal distribution of bullfrogs in the southern springs, Ash Meadows National Wildlife Refuge, Nevada, fall 2007-summer 2008. 


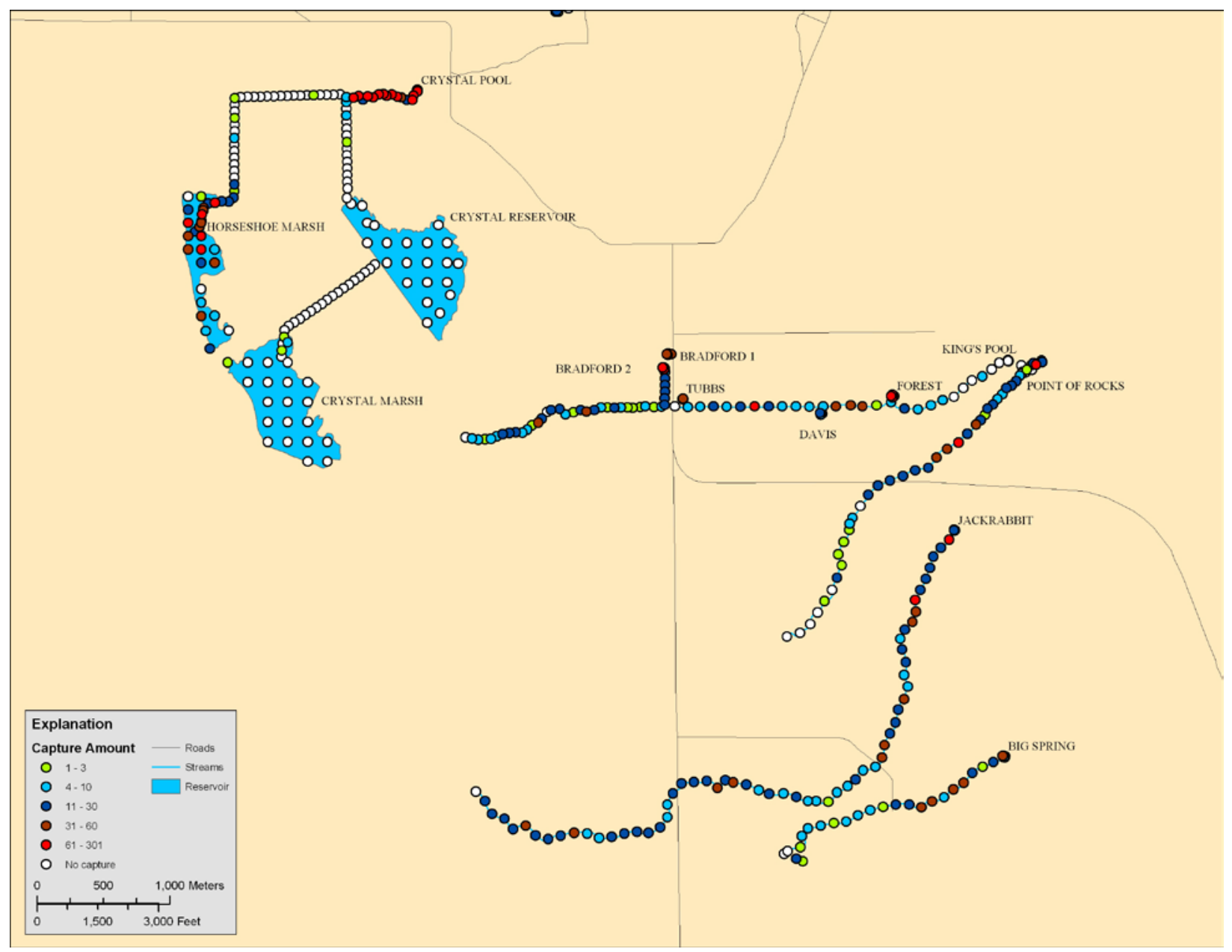

Figure C5. Composite relative abundance and seasonal distribution of crayfish in the southern springs, Ash Meadows National Wildlife Refuge, Nevada, fall 2007-summer 2008. 

Table C1. Species, number, efficiency, and size captured seasonally at the southern springs of the Ash Meadows National Wildlife Refuge, Nevada.

[Spring locations are shown in figures 1 and 2. FL, fork length. Species: CYMI, Ash Meadows Amargosa pupfish; CYPE, Warm Springs Amargosa pupfish; RHON, Ash Meadows speckled dace; GAAF, Mosquitofish; POLA, Sailfin Molly; LECY, Green Sunfish; MISA, Largemouth Bass; ARNI, Convict Cichlid; AMME, Black Bullhead; LICA, Bull Frog; PRCL, Red Swamp Crayfish]

\begin{tabular}{|c|c|c|c|c|c|c|}
\hline \multicolumn{7}{|c|}{ Fall 2007} \\
\hline System & Species & Total catch & Average/trap & $\mathbf{n}$ & $\begin{array}{c}\text { FL } \\
(\min -\max )\end{array}$ & $\begin{array}{c}\text { Average } \pm \text { Standard } \\
\text { Deviation }\end{array}$ \\
\hline \multirow{4}{*}{ Crystal spring-pool } & CYMI & 319 & 53.2 & 55 & $16-40$ & $30 \pm 5$ \\
\hline & GAAF & 66 & 11.0 & 16 & $21-35$ & $27 \pm 3$ \\
\hline & POLA & 1 & 0.2 & 1 & 40 & 40 \\
\hline & PRCL & 70 & 11.7 & 38 & $30-68$ & $52 \pm 9$ \\
\hline \multirow{4}{*}{ Crystal stream } & CYMI & 1423 & 21.9 & 279 & $16-49$ & $31 \pm 6$ \\
\hline & GAAF & 339 & 5.2 & 144 & $18-50$ & $29 \pm 5$ \\
\hline & POLA & 105 & 1.6 & 66 & $17-61$ & $33 \pm 8$ \\
\hline & PRCL & 609 & 9.4 & 213 & $15-76$ & $55 \pm 10$ \\
\hline \multirow{3}{*}{ Crystal reservoir } & CYMI & - & - & - & - & - \\
\hline & LECY & 386 & 11.7 & 156 & $24-220$ & $50 \pm 26$ \\
\hline & MISA & 3 & 0.1 & 3 & $67-91$ & $79 \pm 12$ \\
\hline \multirow{3}{*}{ Crystal stream } & GAAF & 1 & - & 1 & 41 & 41 \\
\hline & LECY & 10 & 0.5 & 10 & $28-66$ & $39 \pm 12$ \\
\hline & PRCL & 1 & - & 1 & $66-66$ & 66 \\
\hline \multirow{5}{*}{ Crystal marsh } & CYMI & 24 & 1.1 & 12 & $16-44$ & $28 \pm 7$ \\
\hline & GAAF & 66 & 3.1 & 10 & $19-30$ & $26 \pm 3$ \\
\hline & LECY & 26 & 0.8 & 26 & $31-165$ & $59 \pm 31$ \\
\hline & MISA & - & - & - & - & - \\
\hline & PRCL & 3 & 0.1 & 3 & $43-48$ & $45 \pm 3$ \\
\hline \multirow{6}{*}{ Horseshoe marsh } & CYMI & 17 & 1.1 & 17 & $18-36$ & $25 \pm 5$ \\
\hline & GAAF & 97 & 6.1 & 61 & $18-45$ & $30 \pm 7$ \\
\hline & POLA & 54 & 3.4 & 30 & $14-55$ & $35 \pm 9$ \\
\hline & LECY & - & - & - & - & - \\
\hline & LICA & 1 & 0.1 & 1 & $150-150$ & 150 \\
\hline & PRCL & 210 & 13.1 & 101 & $27-78$ & $58 \pm 11$ \\
\hline \multirow{2}{*}{$\begin{array}{c}\text { Kings Pool spring- } \\
\text { pool }\end{array}$} & CYMI & 838 & 139.7 & 107 & $17-43$ & $31 \pm 5$ \\
\hline & GAAF & 4 & 0.7 & 4 & $20-36$ & $27 \pm 7$ \\
\hline \multirow{5}{*}{ Kings Pool stream } & CYMI & 179 & 6.4 & 65 & $16-50$ & $34 \pm 7$ \\
\hline & RHON & 1 & . & 1 & 51 & 51 \\
\hline & GAAF & 57 & 2.0 & 52 & $20-47$ & $31 \pm 7$ \\
\hline & POLA & 29 & 1.0 & 29 & $22-50$ & $35 \pm 8$ \\
\hline & PRCL & 80 & 2.9 & 69 & $25-75$ & $57 \pm 11$ \\
\hline \multirow{6}{*}{$\begin{array}{l}\text { Point of Rocks } \\
\text { spring-pool }\end{array}$} & CYMI & 237 & 5.3 & 93 & $13-48$ & $30 \pm 7$ \\
\hline & RHON & - & - & - & - & - \\
\hline & GAAF & 29 & 0.6 & 29 & $23-37$ & $29 \pm 4$ \\
\hline & POLA & 5 & 0.1 & 5 & $23-41$ & $33 \pm 7$ \\
\hline & LICA & - & - & - & - & - \\
\hline & PRCL & 196 & 4.4 & 150 & $15-70$ & $52 \pm 10$ \\
\hline \multirow{3}{*}{$\begin{array}{l}\text { Bradford } 1 \text { spring- } \\
\text { pool }\end{array}$} & RHON & 615 & 102.5 & 120 & $24-75$ & $43 \pm 11$ \\
\hline & GAAF & 125 & 20.8 & 47 & $14-60$ & $30 \pm 7$ \\
\hline & PRCL & 37 & 6.2 & 37 & $27-74$ & $49 \pm 14$ \\
\hline \multirow{3}{*}{ Bradford 1 stream } & RHON & 80 & 40.0 & 10 & $25-51$ & $38 \pm 8$ \\
\hline & GAAF & 93 & 46.5 & 20 & $20-38$ & $28 \pm 5$ \\
\hline & PRCL & 30 & 15.0 & 20 & $24-72$ & $50 \pm 14$ \\
\hline \multirow{5}{*}{$\begin{array}{l}\text { Bradford } 2 \text { spring- } \\
\text { pool }\end{array}$} & RHON & 31 & 5.2 & 31 & $29-54$ & $43 \pm 5$ \\
\hline & GAAF & 358 & 59.7 & 83 & $18-43$ & $26 \pm 5$ \\
\hline & POLA & - & - & - & - & - \\
\hline & LICA & - & - & - & - & - \\
\hline & PRCL & 153 & 25.5 & 89 & $24-65$ & $45 \pm 10$ \\
\hline
\end{tabular}


Table C1. Species, number, efficiency, and size captured seasonally at the southern springs of the Ash Meadows National Wildlife Refuge, Nevada.-Continued

Fall 2007

\begin{tabular}{|c|c|c|c|c|c|c|}
\hline System & Species & Total catch & Average/trap & $\mathbf{n}$ & $\begin{array}{c}\mathrm{FL} \\
(\min -\max )\end{array}$ & $\begin{array}{c}\text { Average } \pm \text { Standard } \\
\text { Deviation }\end{array}$ \\
\hline \multirow{6}{*}{ Bradford 2 stream } & CYMI & 15 & 0.5 & 15 & $26-45$ & $35 \pm 5$ \\
\hline & RHON & 95 & 2.9 & 77 & $28-79$ & $46 \pm 9$ \\
\hline & GAAF & 163 & 4.9 & 86 & $19-52$ & $36 \pm 7$ \\
\hline & POLA & 22 & 0.7 & 20 & $26-53$ & $39 \pm 9$ \\
\hline & LICA & 1 & - & 0 & - & - \\
\hline & PRCL & 141 & 4.3 & 125 & $10-75$ & $53 \pm 14$ \\
\hline \multirow{5}{*}{ Forest spring-pool } & CYMI & 1 & 0.2 & 1 & 33 & 33 \\
\hline & RHON & 5 & 0.8 & 5 & $54-69$ & $58 \pm 6$ \\
\hline & GAAF & 462 & 77.0 & 60 & $18-51$ & $29 \pm 7$ \\
\hline & POLA & 9 & 1.5 & 9 & $20-47$ & $29 \pm 8$ \\
\hline & PRCL & 140 & 23.3 & 50 & $29-89$ & $53 \pm 12$ \\
\hline \multirow{2}{*}{ Tubbs spring-pool } & LICA & 4 & 0.7 & 4 & $71-100$ & $82 \pm 13$ \\
\hline & PRCL & 6 & 1.0 & 6 & $67-74$ & $71 \pm 3$ \\
\hline \multirow{3}{*}{ Davis spring-pool } & GAAF & 119 & 19.8 & 50 & $21-47$ & $34 \pm 8$ \\
\hline & AMME & 8 & 1.3 & 8 & $85-97$ & $89 \pm 4$ \\
\hline & PRCL & 5 & 0.8 & 5 & $55-75$ & $61 \pm 8$ \\
\hline \multirow{5}{*}{$\begin{array}{c}\text { Jackrabbit spring- } \\
\text { pool }\end{array}$} & CYMI & 786 & 131.0 & 55 & $20-55$ & $32 \pm 10$ \\
\hline & RHON & 19 & 3.2 & 19 & $39-55$ & $47 \pm 5$ \\
\hline & GAAF & 55 & 9.2 & 30 & $22-38$ & $30 \pm 5$ \\
\hline & POLA & 69 & 11.5 & 36 & $18-54$ & $35 \pm 10$ \\
\hline & PRCL & 25 & 4.2 & 23 & $30-62$ & $44 \pm 10$ \\
\hline \multirow{6}{*}{ Jackrabbit stream } & CYMI & 79 & 1.3 & 60 & $21-55$ & $39 \pm 7$ \\
\hline & RHON & 510 & 8.5 & 353 & $22-83$ & $50 \pm 12$ \\
\hline & GAAF & 121 & 2.0 & 83 & $21-48$ & $33 \pm 7$ \\
\hline & POLA & 36 & 0.6 & 34 & $18-56$ & $37 \pm 10$ \\
\hline & LICA & - & - & - & - & - \\
\hline & PRCL & 291 & 4.9 & 199 & $16-72$ & $54 \pm 10$ \\
\hline \multirow{6}{*}{$\begin{array}{l}\text { Big Spring spring- } \\
\text { pool }\end{array}$} & CYMI & 157 & 26.2 & 36 & 13-39 & $25 \pm 6$ \\
\hline & GAAF & 2 & 0.3 & 2 & $21-24$ & $23 \pm 2$ \\
\hline & POLA & 40 & 6.7 & 33 & $15-45$ & $29 \pm 10$ \\
\hline & MISA & - & - & - & - & - \\
\hline & LICA & - & - & - & - & - \\
\hline & PRCL & - & - & - & - & - \\
\hline \multirow{5}{*}{ Big Spring stream } & CYMI & 253 & 11.5 & 60 & $22-48$ & $35 \pm 7$ \\
\hline & GAAF & 14 & 0.6 & 14 & $25-51$ & $32 \pm 8$ \\
\hline & POLA & 42 & 1.9 & 41 & $16-62$ & $34 \pm 10$ \\
\hline & LICA & 6 & 0.3 & 6 & $37-55$ & $46 \pm 7$ \\
\hline & PRCL & 117 & 5.3 & 97 & $24-73$ & $50 \pm 12$ \\
\hline
\end{tabular}


Table C1. Species, number, efficiency, and size captured seasonally at the southern springs of the Ash Meadows National Wildlife Refuge, Nevada.-Continued

\begin{tabular}{|c|c|c|c|c|c|c|}
\hline \multicolumn{7}{|c|}{ Winter 2008} \\
\hline System & Species & Total catch & Average/trap & $\mathbf{n}$ & $\begin{array}{c}\mathrm{FL} \\
(\min -\max )\end{array}$ & $\begin{array}{c}\text { Average } \pm \text { Standard } \\
\text { Deviation }\end{array}$ \\
\hline \multirow{4}{*}{ Crystal spring-pool } & CYMI & 594 & 99.0 & 106 & $16-39$ & $28 \pm 4$ \\
\hline & GAAF & 31 & 5.2 & 24 & $20-32$ & $26 \pm 3$ \\
\hline & POLA & - & - & - & - & - \\
\hline & PRCL & 68 & 11.3 & 68 & $21-53$ & $39 \pm 6$ \\
\hline \multirow{4}{*}{ Crystal stream } & CYMI & 1099 & 21.1 & 271 & $18-48$ & $32 \pm 6$ \\
\hline & GAAF & 318 & 6.1 & 95 & $20-41$ & $28 \pm 4$ \\
\hline & POLA & 17 & 0.3 & 16 & $24-65$ & $37 \pm 10$ \\
\hline & PRCL & 639 & 12.3 & 228 & $22-75$ & $49 \pm 10$ \\
\hline \multirow{3}{*}{ Crystal reservoir } & CYMI & - & - & - & - & - \\
\hline & LECY & 4 & 0.1 & 4 & 35-185 & $75 \pm 73$ \\
\hline & MISA & - & - & - & - & - \\
\hline \multirow{3}{*}{ Crystal stream } & GAAF & - & - & - & - & - \\
\hline & LECY & 5 & 0.3 & 5 & $52-98$ & $72 \pm 19$ \\
\hline & PRCL & 1 & 0.1 & 1 & 38 & 38 \\
\hline \multirow{5}{*}{ Crystal marsh } & CYMI & 14 & 0.7 & 11 & $16-30$ & $25 \pm 4$ \\
\hline & GAAF & 5 & 0.3 & 5 & $21-33$ & $29 \pm 5$ \\
\hline & LECY & 4 & 0.1 & 4 & 54-163 & $86 \pm 52$ \\
\hline & MISA & - & - & - & - & - \\
\hline & PRCL & 1 & 0.1 & 1 & 34 & 34 \\
\hline \multirow{6}{*}{ Horseshoe marsh } & CYMI & 27 & 1.6 & 27 & $18-51$ & $29 \pm 8$ \\
\hline & GAAF & 18 & 1.1 & 18 & $23-32$ & $28 \pm 3$ \\
\hline & POLA & 34 & 2.0 & 23 & $25-51$ & $37 \pm 7$ \\
\hline & LECY & 1 & 0.1 & 1 & 61 & 61 \\
\hline & LICA & 11 & 0.6 & 11 & $41-100$ & $79 \pm 15$ \\
\hline & PRCL & 81 & 4.8 & 68 & $25-78$ & $54 \pm 13$ \\
\hline \multirow{2}{*}{$\begin{array}{c}\text { Kings Pool spring- } \\
\text { pool }\end{array}$} & CYMI & 531 & 88.5 & 108 & $16-49$ & $30 \pm 7$ \\
\hline & GAAF & 16 & 2.7 & 16 & $21-36$ & $29 \pm 4$ \\
\hline \multirow{5}{*}{ Kings Pool stream } & CYMI & 106 & 3.8 & 65 & $21-47$ & $34 \pm 6$ \\
\hline & RHON & - & - & - & - & - \\
\hline & GAAF & 35 & 1.3 & 35 & $21-50$ & $31 \pm 7$ \\
\hline & POLA & 12 & 0.4 & 12 & $24-56$ & $37 \pm 11$ \\
\hline & PRCL & 47 & 1.7 & 47 & $25-67$ & $46 \pm 11$ \\
\hline \multirow{6}{*}{$\begin{array}{l}\text { Point of Rocks } \\
\text { spring-pool }\end{array}$} & CYMI & 179 & 4.2 & 56 & $13-43$ & $31 \pm 7$ \\
\hline & RHON & 5 & 0.1 & 5 & $43-58$ & $51 \pm 6$ \\
\hline & GAAF & 91 & 2.1 & 62 & $16-45$ & $30 \pm 6$ \\
\hline & POLA & 7 & 0.2 & 6 & $28-46$ & $35 \pm 7$ \\
\hline & LICA & 1 & - & 0 & - & - \\
\hline & PRCL & 91 & 2.1 & 85 & $22-65$ & $49 \pm 10$ \\
\hline \multirow{3}{*}{$\begin{array}{l}\text { Bradford } 1 \text { spring- } \\
\text { pool }\end{array}$} & RHON & 694 & 115.7 & 125 & $25-72$ & $41 \pm 10$ \\
\hline & GAAF & 447 & 74.5 & 85 & $20-57$ & $29 \pm 7$ \\
\hline & PRCL & 15 & 2.5 & 15 & $28-68$ & $51 \pm 12$ \\
\hline \multirow{3}{*}{ Bradford 1 stream } & RHON & 77 & 38.5 & 15 & $35-61$ & $49 \pm 8$ \\
\hline & GAAF & 78 & 39.0 & 21 & $21-42$ & $32 \pm 7$ \\
\hline & PRCL & 18 & 9.0 & 14 & $41-67$ & $54 \pm 7$ \\
\hline \multirow{5}{*}{$\begin{array}{l}\text { Bradford } 2 \text { spring- } \\
\text { pool }\end{array}$} & RHON & 9 & 1.5 & 9 & $41-54$ & $47 \pm 5$ \\
\hline & GAAF & 390 & 65.0 & 100 & $18-41$ & $27 \pm 4$ \\
\hline & POLA & 141 & 23.5 & 65 & $22-52$ & $34 \pm 5$ \\
\hline & LICA & - & - & - & - & - \\
\hline & PRCL & 52 & 8.7 & 47 & $21-67$ & $39 \pm 9$ \\
\hline
\end{tabular}


Table C1. Species, number, efficiency, and size captured seasonally at the southern springs of the Ash Meadows National Wildlife Refuge, Nevada.-Continued

\begin{tabular}{|c|c|c|c|c|c|c|}
\hline \multicolumn{7}{|c|}{ Winter 2008} \\
\hline System & Species & Total catch & Average/trap & $\mathbf{n}$ & $\begin{array}{c}\mathrm{FL} \\
(\min -\max )\end{array}$ & $\begin{array}{c}\text { Average } \pm \text { Standard } \\
\text { Deviation }\end{array}$ \\
\hline \multirow{6}{*}{ Bradford 2 stream } & CYMI & 3 & 0.1 & 3 & $37-42$ & $40 \pm 3$ \\
\hline & RHON & 94 & 2.5 & 70 & $37-80$ & $51 \pm 9$ \\
\hline & GAAF & 130 & 3.4 & 87 & $16-52$ & $35 \pm 8$ \\
\hline & POLA & 14 & 0.4 & 14 & $32-61$ & $44 \pm 7$ \\
\hline & LICA & 1 & - & 1 & 80 & 80 \\
\hline & PRCL & 100 & 2.6 & 98 & $18-73$ & $50 \pm 15$ \\
\hline \multirow{5}{*}{ Forest spring-pool } & CYMI & 2 & 0.3 & 2 & 39-49 & $44 \pm 7$ \\
\hline & RHON & 7 & 1.2 & 7 & $50-71$ & $60 \pm 9$ \\
\hline & GAAF & 397 & 66.2 & 113 & $17-53$ & $28 \pm 8$ \\
\hline & POLA & 15 & 2.5 & 15 & $20-45$ & $29 \pm 8$ \\
\hline & PRCL & 45 & 7.5 & 44 & $21-75$ & $52 \pm 12$ \\
\hline \multirow{2}{*}{ Tubbs spring-pool } & LICA & - & - & - & - & - \\
\hline & PRCL & 16 & 2.7 & 16 & $37-75$ & $55 \pm 12$ \\
\hline \multirow{3}{*}{ Davis spring-pool } & GAAF & 1 & 0.2 & 1 & 31 & 31 \\
\hline & AMME & - & - & - & - & - \\
\hline & PRCL & 4 & 0.7 & 4 & $50-69$ & $62 \pm 9$ \\
\hline \multirow{5}{*}{$\begin{array}{c}\text { Jackrabbit spring- } \\
\text { pool }\end{array}$} & CYMI & 726 & 121.0 & 118 & $18-49$ & $31 \pm 7$ \\
\hline & RHON & 21 & 3.5 & 21 & $44-55$ & $50 \pm 3$ \\
\hline & GAAF & 52 & 8.7 & 52 & $21-49$ & $30 \pm 6$ \\
\hline & POLA & 35 & 5.8 & 27 & $21-42$ & $29 \pm 5$ \\
\hline & PRCL & 24 & 4.0 & 24 & $31-66$ & $46 \pm 10$ \\
\hline \multirow{6}{*}{ Jackrabbit stream } & CYMI & 169 & 2.8 & 86 & $25-53$ & $40 \pm 7$ \\
\hline & RHON & 645 & 10.8 & 405 & $25-82$ & $51 \pm 13$ \\
\hline & GAAF & 56 & 0.9 & 51 & $24-55$ & $34 \pm 7$ \\
\hline & POLA & 3 & 0.1 & 3 & $41-43$ & $42 \pm 1$ \\
\hline & LICA & - & - & - & - & - \\
\hline & PRCL & 295 & 4.9 & 231 & $15-71$ & $44 \pm 13$ \\
\hline \multirow{6}{*}{$\begin{array}{l}\text { Big Spring spring- } \\
\text { pool }\end{array}$} & CYMI & 416 & 69.3 & 89 & $18-42$ & $26 \pm 4$ \\
\hline & GAAF & 13 & 2.2 & 13 & $18-27$ & $22 \pm 3$ \\
\hline & POLA & 9 & 1.5 & 9 & $20-35$ & $24 \pm 5$ \\
\hline & MISA & - & - & - & - & - \\
\hline & LICA & 15 & 2.5 & 15 & $42-65$ & $52 \pm 7$ \\
\hline & PRCL & 1 & 0.2 & 1 & $25-25$ & 25 \\
\hline \multirow{5}{*}{ Big Spring stream } & CYMI & 206 & 9.8 & 70 & $19-55$ & $32 \pm 8$ \\
\hline & GAAF & 36 & 1.7 & 36 & $20-51$ & $29 \pm 7$ \\
\hline & POLA & 8 & 0.4 & 8 & $25-41$ & $30 \pm 5$ \\
\hline & LICA & 14 & 0.7 & 12 & $35-71$ & $55 \pm 12$ \\
\hline & PRCL & 92 & 4.4 & 79 & $28-62$ & $45 \pm 9$ \\
\hline
\end{tabular}


Table C1. Species, number, efficiency, and size captured seasonally at the southern springs of the Ash Meadows National Wildlife Refuge, Nevada.-Continued

\begin{tabular}{|c|c|c|c|c|c|c|}
\hline \multicolumn{7}{|c|}{ Spring 2008} \\
\hline System & Species & Total catch & Average/trap & $\mathbf{n}$ & $\begin{array}{c}\mathrm{FL} \\
(\min -\max )\end{array}$ & $\begin{array}{c}\text { Average } \pm \text { Standard } \\
\text { Deviation }\end{array}$ \\
\hline \multirow{4}{*}{ Crystal spring-pool } & CYMI & 564 & 94.0 & 108 & $20-45$ & $29 \pm 5$ \\
\hline & GAAF & 21 & 3.5 & 21 & $23-35$ & $28 \pm 4$ \\
\hline & POLA & 2 & 0.3 & 2 & $36-38$ & $37 \pm 1$ \\
\hline & PRCL & 24 & 4.0 & 24 & $39-59$ & $49 \pm 5$ \\
\hline \multirow{4}{*}{ Crystal stream } & CYMI & 646 & 17.5 & 241 & $18-52$ & $33 \pm 6$ \\
\hline & GAAF & 190 & 5.1 & 108 & $18-47$ & $30 \pm 6$ \\
\hline & POLA & 11 & 0.3 & 10 & $16-62$ & $33 \pm 14$ \\
\hline & PRCL & 402 & 10.9 & 169 & $25-75$ & $51 \pm 9$ \\
\hline \multirow{3}{*}{ Crystal reservoir } & CYMI & 5 & 0.2 & 5 & $30-38$ & $32 \pm 3$ \\
\hline & LECY & 9 & 0.3 & 9 & $38-68$ & $52 \pm 9$ \\
\hline & MISA & 5 & 0.1 & 5 & $231-287$ & $271 \pm 23$ \\
\hline \multirow{3}{*}{ Crystal stream } & GAAF & - & - & - & - & - \\
\hline & LECY & - & - & - & - & - \\
\hline & PRCL & - & - & - & - & - \\
\hline \multirow{5}{*}{ Crystal marsh } & CYMI & 6 & 0.3 & 6 & $18-28$ & $23 \pm 3$ \\
\hline & GAAF & - & - & - & - & - \\
\hline & LECY & 27 & 0.9 & 27 & 34-175 & $80 \pm 38$ \\
\hline & MISA & 16 & 0.6 & 16 & 209-315 & $256 \pm 24$ \\
\hline & PRCL & 2 & 0.1 & 2 & $47-52$ & $50 \pm 4$ \\
\hline \multirow{6}{*}{ Horseshoe marsh } & CYMI & 72 & 4.8 & 30 & $20-42$ & $32 \pm 6$ \\
\hline & GAAF & 16 & 1.1 & 16 & $24-39$ & $31 \pm 5$ \\
\hline & POLA & 19 & 1.3 & 19 & $24-47$ & $33 \pm 6$ \\
\hline & LECY & 5 & 0.3 & 5 & $64-74$ & $70 \pm 4$ \\
\hline & LICA & 2 & 0.1 & 2 & $50-72$ & $61 \pm 16$ \\
\hline & PRCL & 174 & 11.6 & 98 & $30-75$ & $57 \pm 10$ \\
\hline \multirow{2}{*}{$\begin{array}{c}\text { Kings Pool spring- } \\
\text { pool }\end{array}$} & CYMI & 653 & 108.8 & 108 & $18-44$ & $29 \pm 6$ \\
\hline & GAAF & 1 & 0.2 & 1 & 34 & 34 \\
\hline \multirow{5}{*}{ Kings Pool stream } & CYMI & 170 & 6.1 & 93 & $28-51$ & $35 \pm 5$ \\
\hline & RHON & - & - & - & - & - \\
\hline & GAAF & 29 & 1.0 & 29 & $19-44$ & $30 \pm 7$ \\
\hline & POLA & 29 & 1.0 & 26 & $20-41$ & $33 \pm 5$ \\
\hline & PRCL & 78 & 2.8 & 73 & $33-71$ & $54 \pm 10$ \\
\hline \multirow{6}{*}{$\begin{array}{l}\text { Point of Rocks } \\
\text { spring-pool }\end{array}$} & CYMI & 227 & 5.5 & 152 & $17-44$ & $31 \pm 6$ \\
\hline & RHON & 2 & - & 2 & $38-65$ & $52 \pm 19$ \\
\hline & GAAF & 49 & 1.2 & 42 & $20-47$ & $31 \pm 5$ \\
\hline & POLA & 6 & 0.1 & 6 & $32-42$ & $38 \pm 3$ \\
\hline & LICA & - & - & - & - & - \\
\hline & PRCL & 148 & 3.6 & 112 & $19-73$ & $52 \pm 12$ \\
\hline \multirow{3}{*}{$\begin{array}{l}\text { Bradford } 1 \text { spring- } \\
\text { pool }\end{array}$} & RHON & 383 & 63.8 & 100 & $37-72$ & $46 \pm 7$ \\
\hline & GAAF & 51 & 8.5 & 46 & $20-45$ & $34 \pm 7$ \\
\hline & PRCL & 29 & 4.8 & 29 & $32-70$ & $50 \pm 11$ \\
\hline \multirow{3}{*}{ Bradford 1 stream } & RHON & - & - & - & - & - \\
\hline & GAAF & 261 & 130.5 & 21 & $24-60$ & $35 \pm 9$ \\
\hline & PRCL & 13 & 6.5 & 10 & $42-70$ & $59 \pm 8$ \\
\hline \multirow{5}{*}{$\begin{array}{l}\text { Bradford } 2 \text { spring- } \\
\text { pool }\end{array}$} & RHON & - & - & - & - & - \\
\hline & GAAF & - & - & - & - & - \\
\hline & POLA & - & - & - & - & - \\
\hline & LICA & 1 & 0.2 & 0 & - & - \\
\hline & PRCL & 95 & 15.8 & 68 & $26-68$ & $44 \pm 10$ \\
\hline
\end{tabular}


Table C1. Species, number, efficiency, and size captured seasonally at the southern springs of the Ash Meadows National Wildlife Refuge, Nevada.-Continued

\begin{tabular}{|c|c|c|c|c|c|c|}
\hline \multicolumn{7}{|c|}{ Spring 2008} \\
\hline System & Species & Total catch & Average/trap & $\mathbf{n}$ & $\begin{array}{c}\mathrm{FL} \\
(\min -\max )\end{array}$ & $\begin{array}{c}\text { Average } \pm \text { Standard } \\
\text { Deviation }\end{array}$ \\
\hline \multirow{6}{*}{ Bradford 2 stream } & CYMI & 14 & 0.4 & 14 & $25-42$ & $33 \pm 5$ \\
\hline & RHON & 79 & 2.1 & 57 & $31-79$ & $48 \pm 10$ \\
\hline & GAAF & 88 & 2.3 & 61 & $20-52$ & $35 \pm 6$ \\
\hline & POLA & 1 & - & 1 & 35 & 35 \\
\hline & LICA & 2 & 0.1 & 2 & $71-101$ & $86 \pm 21$ \\
\hline & PRCL & 85 & 2.2 & 85 & $21-75$ & $54 \pm 12$ \\
\hline \multirow{5}{*}{ Forest spring-pool } & CYMI & 55 & 9.2 & 55 & $32-57$ & $42 \pm 6$ \\
\hline & RHON & 7 & 1.2 & 7 & $42-70$ & $54 \pm 9$ \\
\hline & GAAF & 287 & 47.8 & 83 & $16-47$ & $28 \pm 7$ \\
\hline & POLA & 1 & 0.2 & 1 & 37 & 37 \\
\hline & PRCL & 152 & 25.3 & 110 & $17-68$ & $49 \pm 10$ \\
\hline \multirow{2}{*}{ Tubbs spring-pool } & LICA & 2 & 0.3 & 2 & $105-115$ & $110 \pm 7$ \\
\hline & PRCL & 49 & 8.2 & 49 & $20-74$ & $54 \pm 10$ \\
\hline \multirow{3}{*}{ Davis spring-pool } & GAAF & 359 & 59.8 & 112 & $22-53$ & $38 \pm 7$ \\
\hline & AMME & 2 & 0.3 & 2 & 95-103 & $99 \pm 6$ \\
\hline & PRCL & 19 & 3.2 & 19 & $28-73$ & $47 \pm 14$ \\
\hline \multirow{5}{*}{$\begin{array}{c}\text { Jackrabbit spring- } \\
\text { pool }\end{array}$} & CYMI & 761 & 126.8 & 120 & $16-53$ & $31 \pm 7$ \\
\hline & RHON & 31 & 5.2 & 31 & $42-60$ & $50 \pm 5$ \\
\hline & GAAF & 1 & 0.2 & 1 & 40 & 40 \\
\hline & POLA & 75 & 12.5 & 66 & $16-55$ & $38 \pm 6$ \\
\hline & PRCL & 19 & 3.2 & 19 & $31-66$ & $47 \pm 9$ \\
\hline \multirow{6}{*}{ Jackrabbit stream } & CYMI & 176 & 2.9 & 126 & $21-54$ & $38 \pm 7$ \\
\hline & RHON & 659 & 11.0 & 440 & $28-85$ & $51 \pm 11$ \\
\hline & GAAF & 91 & 1.5 & 65 & $20-60$ & $36 \pm 8$ \\
\hline & POLA & 8 & 0.1 & 8 & $25-44$ & $37 \pm 6$ \\
\hline & LICA & 1 & - & 0 & - & - \\
\hline & PRCL & 288 & 4.8 & 231 & $15-77$ & $52 \pm 11$ \\
\hline \multirow{6}{*}{$\begin{array}{l}\text { Big Spring spring- } \\
\text { pool }\end{array}$} & CYMI & 107 & 17.8 & 67 & $15-34$ & $23 \pm 4$ \\
\hline & GAAF & 5 & 0.8 & 5 & $21-25$ & $23 \pm 1$ \\
\hline & POLA & 5 & 0.8 & 5 & $18-28$ & $25 \pm 4$ \\
\hline & MISA & 10 & - & 8 & 235-365 & $277 \pm 43$ \\
\hline & LICA & - & - & - & - & - \\
\hline & PRCL & - & - & - & - & - \\
\hline \multirow{5}{*}{ Big Spring stream } & CYMI & 117 & 6.2 & 61 & $17-51$ & $30 \pm 7$ \\
\hline & GAAF & 30 & 1.6 & 30 & $5-44$ & $32 \pm 8$ \\
\hline & POLA & 18 & 0.9 & 18 & $13-49$ & $32 \pm 12$ \\
\hline & LICA & - & - & - & - & - \\
\hline & PRCL & 120 & 6.3 & 75 & $31-67$ & $52 \pm 9$ \\
\hline
\end{tabular}


Table C1. Species, number, efficiency, and size captured seasonally at the southern springs of the Ash Meadows National Wildlife Refuge, Nevada.-Continued

\begin{tabular}{|c|c|c|c|c|c|c|}
\hline \multicolumn{7}{|c|}{ Summer 2008} \\
\hline System & Species & Total catch & Average/trap & $\mathbf{n}$ & $\begin{array}{c}\mathrm{FL} \\
(\min -\max )\end{array}$ & $\begin{array}{c}\text { Average } \pm \text { Standard } \\
\text { Deviation }\end{array}$ \\
\hline \multirow{4}{*}{ Crystal spring-pool } & CYMI & 554 & 92.3 & 103 & $22-46$ & $32 \pm 5$ \\
\hline & GAAF & 27 & 4.5 & 25 & $17-41$ & $29 \pm 7$ \\
\hline & POLA & - & - & - & - & - \\
\hline & PRCL & 34 & 5.7 & 34 & $41-72$ & $56 \pm 7$ \\
\hline \multirow{4}{*}{ Crystal stream } & CYMI & 958 & 36.8 & 225 & $14-51$ & $30 \pm 7$ \\
\hline & GAAF & 242 & 9.3 & 96 & $18-48$ & $29 \pm 7$ \\
\hline & POLA & 3 & 0.1 & 3 & $22-35$ & $29 \pm 7$ \\
\hline & PRCL & 605 & 23.3 & 150 & $35-72$ & $56 \pm 8$ \\
\hline \multirow{3}{*}{ Crystal reservoir } & CYMI & - & - & - & - & - \\
\hline & LECY & 77 & 2.6 & 77 & $18-216$ & $62 \pm 32$ \\
\hline & MISA & - & - & - & - & - \\
\hline \multirow{3}{*}{ Crystal stream } & GAAF & - & - & - & - & - \\
\hline & LECY & - & - & - & - & - \\
\hline & PRCL & - & - & - & - & - \\
\hline \multirow{5}{*}{ Crystal marsh } & CYMI & 4 & 0.5 & 4 & $22-28$ & $24 \pm 3$ \\
\hline & GAAF & 4 & 0.5 & 4 & $17-23$ & $20 \pm 3$ \\
\hline & LECY & 41 & 3.7 & 39 & $16-167$ & $46 \pm 37$ \\
\hline & MISA & 1 & 0.1 & 1 & 48 & 48 \\
\hline & PRCL & - & - & - & - & - \\
\hline \multirow{6}{*}{ Horseshoe marsh } & CYMI & 12 & 1.2 & 12 & $26-40$ & $35 \pm 5$ \\
\hline & GAAF & 437 & 43.7 & 78 & $16-68$ & $26 \pm 8$ \\
\hline & POLA & 44 & 4.4 & 11 & $21-42$ & $33 \pm 7$ \\
\hline & LECY & - & - & - & - & - \\
\hline & LICA & 37 & 3.7 & 0 & - & - \\
\hline & PRCL & 162 & 16.2 & 69 & $29-72$ & $54 \pm 11$ \\
\hline \multirow{2}{*}{$\begin{array}{c}\text { Kings Pool spring- } \\
\text { pool }\end{array}$} & CYMI & 701 & 116.8 & 100 & $16-46$ & $32 \pm 6$ \\
\hline & GAAF & 2 & 0.3 & 2 & $32-33$ & $33 \pm 1$ \\
\hline \multirow{5}{*}{ Kings Pool stream } & CYMI & 157 & 5.6 & 96 & $23-49$ & $36 \pm 6$ \\
\hline & RHON & - & - & - & - & - \\
\hline & GAAF & 74 & 2.6 & 69 & $15-51$ & $33 \pm 7$ \\
\hline & POLA & 41 & 1.5 & 31 & $21-49$ & $34 \pm 7$ \\
\hline & PRCL & 104 & 3.7 & 92 & $29-72$ & $56 \pm 9$ \\
\hline \multirow{6}{*}{$\begin{array}{l}\text { Point of Rocks } \\
\text { spring-pool }\end{array}$} & CYMI & 234 & 5.9 & 130 & $15-45$ & $31 \pm 5$ \\
\hline & RHON & - & - & - & - & - \\
\hline & GAAF & 119 & 3.0 & 96 & $18-45$ & $30 \pm 6$ \\
\hline & POLA & 3 & 0.1 & 3 & $29-37$ & $32 \pm 4$ \\
\hline & LICA & - & - & - & - & - \\
\hline & PRCL & 198 & 5.0 & 156 & $17-75$ & $53 \pm 12$ \\
\hline \multirow{3}{*}{$\begin{array}{l}\text { Bradford } 1 \text { spring- } \\
\text { pool }\end{array}$} & RHON & 265 & 44.2 & 78 & $34-62$ & $49 \pm 6$ \\
\hline & GAAF & 334 & 55.7 & 88 & $18-56$ & $30 \pm 10$ \\
\hline & PRCL & 29 & 4.8 & 29 & $29-78$ & $53 \pm 14$ \\
\hline \multirow{3}{*}{ Bradford 1 stream } & RHON & 33 & 8.3 & 14 & $50-62$ & $55 \pm 3$ \\
\hline & GAAF & 692 & 173.0 & 40 & $21-55$ & $31 \pm 9$ \\
\hline & PRCL & 26 & 6.5 & 26 & $30-71$ & $55 \pm 11$ \\
\hline \multirow{5}{*}{$\begin{array}{l}\text { Bradford } 2 \text { spring- } \\
\text { pool }\end{array}$} & RHON & 2 & 0.3 & 2 & $33-42$ & $38 \pm 6$ \\
\hline & GAAF & 31 & 5.2 & 30 & $18-46$ & $25 \pm 5$ \\
\hline & POLA & - & - & - & - & - \\
\hline & LICA & - & - & - & - & - \\
\hline & PRCL & 40 & 6.7 & 40 & $25-60$ & $46 \pm 9$ \\
\hline
\end{tabular}


Table C1. Species, number, efficiency, and size captured seasonally at the southern springs of the Ash Meadows National Wildlife Refuge, Nevada.-Continued

\begin{tabular}{|c|c|c|c|c|c|c|}
\hline \multicolumn{7}{|c|}{ Summer 2008} \\
\hline System & Species & Total catch & Average/trap & $\mathrm{n}$ & $\begin{array}{c}\mathrm{FL} \\
(\min -\max )\end{array}$ & $\begin{array}{c}\text { Average } \pm \text { Standard } \\
\text { Deviation }\end{array}$ \\
\hline \multirow{6}{*}{ Bradford 2 stream } & CYMI & 5 & 0.1 & 5 & $22-41$ & $30 \pm 8$ \\
\hline & RHON & 78 & 2.1 & 55 & $27-74$ & $47 \pm 11$ \\
\hline & GAAF & 123 & 3.3 & 79 & $20-51$ & $35 \pm 7$ \\
\hline & POLA & 4 & 0.1 & 4 & $21-40$ & $31 \pm 8$ \\
\hline & LICA & - & - & - & - & - \\
\hline & PRCL & 120 & 3.2 & 117 & $21-71$ & $54 \pm 11$ \\
\hline \multirow{5}{*}{ Forest spring-pool } & CYMI & 42 & 5.3 & 42 & $27-54$ & $41 \pm 7$ \\
\hline & RHON & 1 & 0.1 & 1 & 55 & 55 \\
\hline & GAAF & 415 & 51.9 & 141 & $12-45$ & $26 \pm 6$ \\
\hline & POLA & 4 & 0.5 & 4 & $16-50$ & $33 \pm 14$ \\
\hline & PRCL & 140 & 17.5 & 114 & $25-71$ & $51 \pm 10$ \\
\hline \multirow{2}{*}{ Tubbs spring-pool } & LICA & 8 & 1.3 & 0 & - & - \\
\hline & PRCL & 51 & 8.5 & 51 & $20-78$ & $52 \pm 13$ \\
\hline \multirow{3}{*}{ Davis spring-pool } & GAAF & - & - & - & - & - \\
\hline & AMME & - & - & - & - & - \\
\hline & PRCL & 11 & 1.8 & 11 & $35-58$ & $45 \pm 8$ \\
\hline \multirow{5}{*}{$\begin{array}{c}\text { Jackrabbit spring- } \\
\text { pool }\end{array}$} & CYMI & 512 & 85.3 & 112 & $17-48$ & $34 \pm 7$ \\
\hline & RHON & 23 & 3.8 & 23 & $44-58$ & $50 \pm 4$ \\
\hline & GAAF & 28 & 4.7 & 28 & $18-46$ & $32 \pm 9$ \\
\hline & POLA & 32 & 5.3 & 32 & $17-49$ & $29 \pm 8$ \\
\hline & PRCL & 43 & 7.2 & 43 & $25-72$ & $51 \pm 10$ \\
\hline \multirow{6}{*}{ Jackrabbit stream } & CYMI & 147 & 2.5 & 135 & $15-54$ & $36 \pm 9$ \\
\hline & RHON & 607 & 10.1 & 413 & 21-91 & $55 \pm 12$ \\
\hline & GAAF & 333 & 5.6 & 253 & $20-56$ & $32 \pm 7$ \\
\hline & POLA & 11 & 0.2 & 11 & $22-45$ & $33 \pm 8$ \\
\hline & LICA & - & - & - & - & - \\
\hline & PRCL & 461 & 7.7 & 356 & $27-74$ & $55 \pm 10$ \\
\hline \multirow{6}{*}{$\begin{array}{l}\text { Big Spring spring- } \\
\text { pool }\end{array}$} & CYMI & 213 & 35.5 & 76 & $7-32$ & $21 \pm 4$ \\
\hline & GAAF & 128 & 21.3 & 50 & $10-31$ & $21 \pm 4$ \\
\hline & POLA & 32 & 5.3 & 32 & $11-33$ & $23 \pm 6$ \\
\hline & MISA & - & - & - & - & - \\
\hline & LICA & 8 & 1.3 & 0 & - & - \\
\hline & PRCL & 7 & 1.2 & 7 & $43-65$ & $59 \pm 7$ \\
\hline \multirow{5}{*}{ Big Spring stream } & CYMI & 61 & 3.2 & 61 & $18-42$ & $30 \pm 6$ \\
\hline & GAAF & 109 & 5.7 & 83 & $18-45$ & $30 \pm 7$ \\
\hline & POLA & 44 & 2.3 & 35 & $18-65$ & $30 \pm 11$ \\
\hline & LICA & 2 & 0.1 & 2 & $58-60$ & $59 \pm 1$ \\
\hline & PRCL & 54 & 2.8 & 54 & $30-75$ & $54 \pm 11$ \\
\hline
\end{tabular}


Publishing support provided by the U.S. Geological Survey Publishing Network, Tacoma Publishing Service Center

For more information concerning the research in this report, contact the Director, Western Fisheries Research Center

U.S. Geological Survey

6505 NE 65th Street

Seattle, Washington 98115

http://wfrc.usgs.gov/ 
